\title{
Branching of Singularities for Degenerate Hyperbolic Operators
}

\author{
By \\ Kazuo Amano* and Gen NAKAMURA**
}

\section{§1. Introduction}

Let $t \in[-T, T], \quad x=\left(x_{1}, \cdots, x_{n}\right) \in \mathbb{R}^{n}, \quad D_{t}=(1 / \sqrt{-1}) \partial / \partial t, \quad D_{x}=$ $\left(D_{x_{1}}, \cdots, D_{x_{n}}\right), D_{x_{j}}=(1 / \sqrt{-1}) \partial / \partial x_{j}(1 \leq j \leq n)$ and $P\left(t, X, D_{t}, D_{x}\right)$ be a $m$-th order linear hyperbolic partial differential operator with $C^{\infty}$ coefficients. Consider the Cauchy problem:

$$
\left\{\begin{array}{l}
P u=0 \quad \text { in } \quad(s, T] \times \mathbb{R}^{n}, \\
\left.D_{t}^{h} u\right|_{t=s}=u_{h} \in \mathscr{E}^{\prime}\left(\mathbb{R}^{n}\right) \quad(0 \leq h \leq m-1) .
\end{array}\right.
$$

We assume the principal symbol $P_{m}(t, x, \tau, \xi)$ of $P$ is smoothly factorizable: $P_{m}(\tau, x, \tau, \xi)=\prod_{i=1}^{m}\left(\tau-\tilde{\lambda}_{i}(t, x, \xi)\right), \tilde{\lambda}_{l}(t, x, \xi) \in C^{\infty}([-T, T]$ $\left.\times \boldsymbol{R}^{n} \times\left(\boldsymbol{R}^{n}-\{0\}\right)\right)(1 \leq i \leq m)$. For each $i(1 \leq i \leq m)$, associate the Hamilton vector field $H_{i}$ :

$$
H_{i}=\frac{\partial}{\partial t}+\frac{\partial \tilde{\lambda}_{i}}{\partial t} \frac{\partial}{\partial \tau}-\sum_{j=1}^{m}\left(\frac{\partial \tilde{\lambda}_{i}}{\partial \xi_{j}} \frac{\partial}{\partial x_{j}}-\frac{\partial \tilde{\lambda}_{i}}{\partial x_{j}} \frac{\partial}{\partial \xi_{j}}\right) .
$$

Then it is already well known that the singularities of the solution $u$ of (1.1) propagate along each integral curve $\gamma_{i}$ of $H_{i}$ which starts from a point $\left(s, y, \tilde{\lambda}_{i}(s, y, \eta), \eta\right)$ with $(y, \eta) \in \bigcup_{0 \leq h \leq m-1} W F\left(u_{h}\right)$ as long as they reach a singular point $\sigma$ of the characteristic variety $\{(t, x, \tau, \xi)$; $\left.P_{m}(t, x, \tau, \xi)=0\right\}$. A natural question arises. Namely, how do the singularities propagate after one of these curves reached the singular point $\sigma$. For example, if $\gamma_{i}$ passes the singular point $\sigma$, then there is a case that the singularities bifurcate at $\sigma$ and propagate not only

Communicated by S. Matsuura, June 22, 1982.

* Department of Mathematics, Josai University, Saitama 350-02, Japan.

** Department of Mathematics, Massachusetts Institute of Technology, Cambridge, Massachusetts 02139, U. S. A.

Present address: Department of Mathematics, Josai University, Saitama 350-02, Japan. 
along $\gamma_{i}$ but also along an integral curve of $H_{j}(j \neq i)$ through $\sigma$. Alinhac [1] named this kind of phenomena branching of singularities. He gave a condition of branching of singularities for an operator of the form:

$$
D_{t}^{2}-t^{2} D_{x}^{2}+\pi\left(t, D_{x}\right)
$$

where $x \in \boldsymbol{R}^{1}, \pi\left(t, D_{x}\right)$ is a first order pseudo-differential operator. A typical feature of this operator is that characteristic variety is doubly non-invclutive. Namely, the characteristic variety $\{(t, x, \tau, \xi) ;(\tau-t \xi)$ $(\tau+t \xi)=0\}$ satisfies $\{\tau-t \xi, \tau+t \xi\}=2 \neq 0$ and $d(\tau-t \xi) \wedge d(\tau+t \xi) \neq 0$ for $t=0, \xi \neq 0$, where the bracket stands for Poisson bracket. Ivrii [9] and Hanges [7] studied a more general operator with doubly noninvolutive characteristics. The subprincipal symbol plays an essential role in their condition of branching of singularities. However, for an operator with higher order degeneracy at $t=0$, the condition of non-involutive characteristic does not hold. Taniguchi and Tozaki [22] treated this case and considered the operator of the form:

$$
D_{t}^{2}-t^{2 l} D_{x}^{2}+\sqrt{-1} a t^{l-1} D_{x}
$$

where $2 \leq l \in N, a \in \boldsymbol{R}$. They gave a criterion of the branching and non-branching of singularities in terms of the constants $l$ and $a$. For $l=1$, this operator is included in the class treated by Alinhac [1]. However, restricting to this operator, Taniguchi and Tozaki's results are much more precise and detailed. Also Nakane [14] considered the same operator and discussed the propagation of zeroes in the analytic category as a counter example of Treve's conjecture [23]. Recently, in the analytic category, Oaku [16] generalized the results cf Ivrii [9] and Hanges [7] to a system with doubly non-involutive characteristic. Moreover, in the $C^{\infty}$ category, Shinkai [20] considered a first order pseudo-differential system with higher order degeneracy and obtained results similar to ours. Though the operator which we are going to consider can be converted to his system, it is hard to deduce our results from his results and his method. Actually, we have sharpened his results for single equations. Before pointing out the features of our results, we will briefly explain the results known for higher order single equations with higher order degeneracies and the aims of this paper.

For a higher order equation, there is a result due to Amano [2]. 
The operator considered by him is a special kind of operator which we are going to treat here. The additional condition he assumed is that all the coefficients of the operator $P$ consist of monomial with respect to the variable $t$. He gave a criterion of branching and nonbranching of singularities in terms of the Stokes' multipliers associated to the ordinary differential operator obtained by the Fourier transformation of $P$ with respect to the variable $x$. Also, we note that there is a result due to Amano and Nakamura [3]. Although the assumptions and result are the same as ours, the result is incomplete in the fcllowing point. That is its sufficient condition for branching of singularities cannot be applied for arbitrary initial data.

The aim of this paper is to generalize the result of Amano [2] for a more general class of operators and to complete our previous result. More precisely, we shall give the method of obtaining a countable number of sufficient conditions of branching of singularities in terms of the coefficients of central connection problem for a certain ordinary differential operator $L_{0}$ associated with $P$ and a countable number of functions. These countable number of functions are determined by successive integrations of recurrence relations. Each of these integration process is performed by integration of the ordinary differential operator $L_{0}$. If we simply pick up the condition frcm the first step of the integration process, our sufficient condition of branching of singularities is almost the same as that of Amano [2]. More precisely, Amano's condition implies ours.

In the analytic category, Takasaki [21] has recently illustrated the construction of a fundamental solution for our operator whose principal part depends only on the variable $t$. According to that, the amplitude $\tilde{a}_{i, j}$, etc. given below in Theorem 1 which are flat at $t=0$ do not appear and each amplitude is obtained not asymptotically like curs but as a convergent series whose terms consist of a countable number of functions mentioned above. Thus we believe there is a pcssibility to write down completely the necessary and sufficient condition of branching of singularities if we can rewrite the whole things included in this paper as the way Takasaki [21] did.

As we have mentioned above, a concrete sufficient condition of branching of singularities can be obtained if we can compute the central connection coefficients of ordinary differential operator $L_{0}$ 
explicitly. For $m=2$, we can compute these coefficients by using Laplace transformation and as a special case we can obtain Taniguchi and Tozaki's results. For $m \geq 3$, there is a method due to Okubo [17] how to compute these coefficients. However, his method cannot be applied, because the so called pentagonal condition which is crucial for his method is not satisfied for our operator $L_{0}$ in the case $m \geq 3$.

Finally, let us point out that we have sharpened Shinkai's results in the following points.

(i) Concerning the sufficient condition of branching of singularities, we only require that the Stokes' coefficient does not vanish at a single point. (Compare Corollary 2. 2, Theorem 3.2 and Remark 3. 3 of his to Theorem 2 and its remark of ours.)

(ii) From our method, it is easy to obtain many other sufficient ccnditions which can be expressed in terms of the characteristic roots and the coefficients of the partial differential operator and also the central connection coefficients of the associated ordinary differential operator.

(iii) Our central connection coefficients are associated to the ordinary differential operator whose coefficients are holomorphic with respect to $t$ variable. This is very important from the viewpoint of ccmputability of the central connection coefficients, because the analytic continuation of the solution is crucial for those computations. In fact, by using the analytic continuation, we have computed the central connection coefficients and obtained a concrete sufficient ccndition of the branching of singularities for second order equations.

The rest of this paper is organized as follows. In Section 2 we state our results which consist of three theorems (Theorems 1, 2 and 3). As a preparation of the proof of Theorems 1 and 2, we devote Section 3 to the construction of a parametrix $E_{m}^{-}(t, s)$. Then we prove Theorems 1 and 2 in Section 4 by observing the procedure given in Section 3. Finally in Section 5, we briefly illustrate the method how to compute the central connection coefficients for our operator $L_{0}$ in the case $m=2$ which correspond to the proof of Theorem 3 and give some discussions comparing our results to that of Hanges [7] and Taniguchi-Tozaki [22]. 


\section{§2. Assumptions and Results}

Write $P$ in the form:

$$
\begin{aligned}
P= & P_{m}+P_{m-1}+\cdots+P_{0}, \\
& P_{m-j}\left(t, X, D_{t}, D_{x}\right)=\sum_{i=0}^{m-j} P_{i, j}\left(t, X, D_{x}\right) D_{t}^{m-j-i},
\end{aligned}
$$

where $P_{i, j}(t, x, \xi)$ is a homogeneous polynomial of degree $i$ with respect to $\xi$. For simplicity, we assume all the coefficients of $P$ belongs to the space $B^{\infty}\left([-T, T] \times \mathbb{R}_{x}^{n}\right)$, which is the space of $C^{\infty}$ functions whose derivatives are all bounded on $[-T, T] \times \mathbb{R}_{x}^{n}$.

We assume the following conditions (A. 1)-(A. 3) for $P$ which are invariant under change of variables fixing the $t$ axis.

(A. 1$) P_{m}(t, x, \tau, \xi)$ is smoothly factorizable as follows :

$$
P_{m}(t, x, \tau, \xi)=\prod_{j=1}^{m}\left(\tau-t^{l} \lambda_{j}(t, x, \xi)\right)
$$

where $l \in N$ and $\lambda_{j}(t, x, \xi) \in C^{\infty}\left([-T, T] \times \mathbb{R}_{x}^{n} \times\left(\mathbb{R}_{\xi}^{n}-\{0\}\right)\right)(1 \leq j \leq m)$ are real valued.

(A. 2) There exists a constant $c>0$ such that

$$
\begin{aligned}
& \left|\lambda_{j}(t, x, \xi)-\lambda_{k}(t, x, \xi)\right| \geq c|\xi| \\
& \quad\left(j \neq k,(t, x, \xi) \in[-T, T] \times \mathbb{R}_{x}^{n} \times \mathbb{R}_{\xi}^{n}\right)
\end{aligned}
$$

(A. 3) Each $P_{i, j}(t, x, \xi)(i l \geq j, m-j-i \geq 0)$ has the property:

$$
P_{i, j}(t, x, \xi)=t^{i l-j} \tilde{P}_{i, j}(t, x, \xi)
$$

where $\tilde{P}_{i, j}(t, x, \xi)$ is a homogeneous polynomial of degree $i$ whose coefficients are elements of $B^{\infty}\left([-T, T] \times \mathbb{R}_{x}^{n}\right)$.

In order to state our results we need some notations and definitions.

Definitions (Phase functions and double phase functions). For each $j(1 \leq j \leq m)$, define a phase function $\phi_{j}(t, s, x, \xi)$ as the solution of the Cauchy problem:

$$
\partial \phi_{j} / \partial t-t^{l} \lambda_{j}\left(t, x, \nabla_{x} \phi_{j}\right)=0,\left.\phi_{j}\right|_{t=s}=x \cdot \xi
$$

where $x \cdot \xi=\sum_{j=1}^{n} x_{i} \xi_{i}$ for $x=\left(x_{1}, \cdots, x_{n}\right), \xi=\left(\xi_{1}, \cdots, \xi_{n}\right)$. Also, for each $j, k(1 \leq j, k \leq m)$, define a double phase function $\phi_{j, k}(t, s, x, \xi)$ as the solution of the Cauchy problem: 


$$
\partial \phi_{j, k} / \partial t-t^{l} \lambda_{j}\left(t, x, \nabla_{x} \phi_{j, k}\right)=0,\left.\phi_{j, k}\right|_{t=0}=\phi_{k}(0, s, x, \xi)
$$

Remark. If we denote by $T_{j}(t, s)$ and $T_{j, k}(t, s)$ the homogeneous symplectic transformations corresponding to $\phi_{j}(t, s, x, \xi)$ and $\phi_{j, k}(t, s, x, \xi)$, then we have $T_{j, k}(t, s)=T_{j}(t, 0) \cdot T_{k}(0, s)$.

Notations. (Indices $m_{i}^{ \pm}$related to the growth order of the amplitude of the parametrix.) Put

$$
\mu_{i}(x, \xi)=-H_{i}(x, \xi) / G_{i}(x, \xi)
$$

with

$$
\left\{\begin{aligned}
G_{i}(x, \xi)= & \sum_{j=0}^{m-1}(m-j) \lambda_{i}(0, x, \xi)^{m-j-1} \tilde{P}_{j, 0}(0, x, \xi) \\
H_{i}(x, \xi)= & \frac{l}{2} \sum_{j=0}^{m-2}(m-j)(m-j-1) \lambda_{i}(0, x, \xi)^{m-j-1} \tilde{P}_{j, 0}(0, x, \xi) \\
& +\sqrt{-1} \sum_{j=0}^{m-1} \lambda_{i}(0, x, \xi)^{m-j-1} \tilde{P}_{j, 1}(0, x, \xi) .
\end{aligned}\right.
$$

Then define $m_{i}^{ \pm}$by

$$
m_{i}^{ \pm}=\sup _{(x, \xi)}\left\{\operatorname{Re}\left( \pm \mu_{i}(x, \xi)\right)\right\}
$$

Definitions (Central connection coefficients). Set

$$
L_{0}=\sum_{j=0}^{m} \sum_{\substack{i l \geq j \\ m-i-j \geq 0}} t^{i l-j} \tilde{P}_{i, j}(0, x, \xi) D_{t}^{m-j-i} .
$$

Let

$$
\exp \left(\sqrt{-1}(l+1)^{-1} t^{l+1} \lambda_{i}(0, x, \xi)\right) V_{i}^{ \pm}(t, x, \xi) \quad(1 \leq i \leq m)
$$

be a fundamental system of solutions of $L_{0}$ in $\pm t>0$ with the property:

$$
\begin{gathered}
V_{i}^{ \pm}(t, x, \xi) \simeq e_{i}^{*}(t, x, \xi)=t^{\mu_{i}(x, \xi)} \sum_{r=0}^{\infty} e_{i, r}(x, \xi) t^{-r} \\
\text { as } t \rightarrow \pm \infty,
\end{gathered}
$$

where $e_{i, 0}(x, \xi) \equiv 1$ and the symbol " $\simeq$ " denotes the asymptotic expansion uniform with respect to the parameters $x \in \boldsymbol{R}^{n}, \xi(|\xi|=1)$ which is also valid for the derivatives of $V_{i}^{ \pm}(t, x, \xi)$. The asymptotic series for the derivatives of $V_{i}^{ \pm}$are obtained by differentiating $e_{i}^{*}$ formally.*

We also define $V_{\overline{j, i-1}}^{-}(t, x, \xi)$ and $\tilde{V}_{\overline{j, i-1}}^{-}(t, x, \xi)$ by

* The existence of $V_{\imath}^{ \pm}(t, x, \xi)$ can be proved in the same way as Lemma 3. 9 of NakamuraUryu [13]. 


$$
\text { (2. 7) } \begin{aligned}
& V_{j, i-1}^{-}(t, x, \xi)= \exp \left(-\sqrt{-1}(l+1)^{-1} t^{l+1} \lambda_{j}(0, x, \xi)\right) \\
& \times D_{t}^{i-1}\left(\exp \left(\sqrt{-1}(l+1)^{-1} t^{l+1} \lambda_{j}(0, x, \xi)\right) V_{j}^{-}(t, x, \xi)\right) \\
&(2.8) \quad \widetilde{V}_{j, i-1}^{-}(t, x, \xi)=\text { the }(i, j) \text {-cofactor of matrix } \\
&\left(\begin{array}{c}
i \downarrow 0, \cdots, m-1 \\
\left.V_{j, i}^{-}(t, x, \xi): \begin{array}{l}
i \\
j \rightarrow 1, \cdots, m
\end{array}\right) .
\end{array}\right.
\end{aligned}
$$

Furthermore we define $U_{i}(t, x, \xi)$ as a solution of the Cauchy problem:

$$
L_{0} U_{i}=0,\left.D_{t}^{h} U_{i}\right|_{t=0}=\delta_{h, i-1}(0 \leq h \leq m-1),
$$

where $\delta_{h, i}$ denotes Kronecker's delta. Then the central connection coefficients are the coefficients of the linear relation between the pair of the fundamental systems of solutions $U_{i}(t, x, \xi)(1 \leq i \leq m)$ and $\exp \left(\sqrt{-1}(l+1)^{-1} t^{l+1} \lambda_{i}(0, x, \xi)\right) V_{i}^{ \pm}(t, x, \xi)(1 \leq i \leq m)$. Namely,

$$
\begin{aligned}
U_{i}(t, x, \xi) & =\sum_{j=1}^{m} \exp \left(\sqrt{-1}(l+1)^{-1} t^{l+1} \lambda_{j}(0, x, \xi)\right) \\
& \times T_{ \pm}^{(i, j)}(x, \xi) V_{j}^{ \pm}(t, x, \xi) \text { in } \pm t>0
\end{aligned}
$$

for $1 \leq i \leq m$. In addition we define $\widetilde{T}_{-}^{(i, j)}(x, \xi)$ as the $(i, j)$-cofactor of matrix

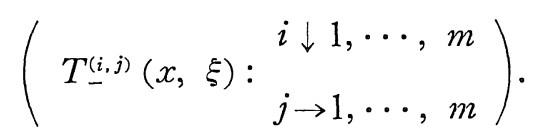

Definition (Symbol classes). Let $\mu, k, \lambda \in \boldsymbol{R}$.

(1) $a(t, s, x, \xi)$ belongs to the symbol class $S^{ \pm}[\mu]$ if $a(t, s, x, \xi)$ is $C^{\infty}$ in $\left\{0 \leq \pm t \leq T_{0}\right\} \times\left\{0 \leq \pm s \leq T_{0}\right\} \times \boldsymbol{R}_{x}^{n} \times\left(\boldsymbol{R}_{\xi}^{n}-\{0\}\right)$ and satisfies the following property: For any $p, q \in \boldsymbol{Z}_{+}, \alpha, \beta \in \boldsymbol{Z}_{+}^{n}$, there exists $c>0$ such that

$$
\left|D_{t}^{\phi} D_{s}^{q} D_{x}^{\alpha} D_{\xi}^{\beta} a(t, s, x, \xi)\right| \leq c(1+|\xi|)^{\mu-|\beta|} \quad(|\xi| \geq 1) .
$$

(2) $a(t, x, \xi)$ belongs to the symbol class $\tilde{S}^{ \pm}[\mu, \kappa]$ if $a(t, x, \xi)$ is $C^{\infty}$ in $\left\{0 \leq \pm t \leq T_{0}\right\} \times \boldsymbol{R}_{x}^{n} \times\left(\boldsymbol{R}_{\xi}^{n}-\{0\}\right)$ and satisfies the following property: For any $p \in \boldsymbol{Z}_{+}, \alpha, \beta \in \boldsymbol{Z}_{+}^{n}$, there exists constant $c>0$ such that

$\left|D_{t}^{p} D_{x}^{\alpha} D_{\xi}^{\beta} a(t, x, \xi)\right| \leq c(1+|\xi|)^{\mu-|\beta|}\left(|\xi|^{-1}+|t|^{l+1}\right)^{(x-p) /(l+1)}(|\xi| \geq 1)$.

(3) $a(t, s, x, \xi)$ belongs to the symbol class $\tilde{S}^{ \pm}[\mu, \kappa, \lambda]$ if $a(t, s, x, \xi)$ is $C^{\infty}$ in $\left\{0 \leq \pm t \leq T_{0}\right\} \times\left\{0 \leq \pm s \leq T_{0}\right\} \times \boldsymbol{R}_{x}^{n} \times\left(\boldsymbol{R}_{\xi}^{n}-\{0\}\right)$ and satisfies the following property: For any $p, q \in \boldsymbol{Z}_{+}, \alpha, \beta \in \mathbb{Z}_{-}^{n}$, there exists $c>0$ such that 


$$
\begin{aligned}
& \left|D_{t}^{\phi} D_{s}^{q} D_{t}^{\alpha} D_{\xi}^{\beta} a(t, s, x, \xi)\right| \leq c(1+|\xi|)^{\mu-|\beta|} \\
& \quad\left(|\xi|^{-1}+|t|^{l+1}\right)^{\frac{\kappa-p}{l+1}}\left(|\xi|^{-1}+|s|^{l+1}\right)^{\frac{\lambda-q}{l+1}}(|\xi| \geq 1) .
\end{aligned}
$$

(4) $a(t, s, x, \xi)$ belongs to the symbol class $\widetilde{S}^{-}[\mu, \kappa]$ if $a(t, s, x, \xi)$ is $C^{\infty}$ in $\left\{(t, s):-T_{0} \leq s \leq t \leq 0\right\} \times \boldsymbol{R}_{x}^{n} \times\left(\boldsymbol{R}_{\xi}^{n}-\{0\}\right)$ and satisfies the following property: For any $p, q, \in \mathbb{Z}_{+}, \alpha, \beta \in \mathbb{Z}_{+}^{n}$, there exists $c>0$ such that

$$
\left|D_{t}^{p} D_{s}^{q} D_{x}^{\alpha} D_{\xi}^{\beta} a(t, s, x, \xi)\right| \leq c|t|^{\kappa}(1+|\xi|)^{\mu-|\beta|} \quad(|\xi| \geq 1) .
$$

Here $\boldsymbol{Z}_{+}$denotes the set of non-negative integers and $\mathbb{Z}_{+}^{n}=\{\alpha$ $\left.=\left(\alpha_{1}, \cdots, \alpha_{n}\right) ; \alpha_{i} \in Z_{+}(1 \leq i \leq n)\right\}$. Moreover, we define the symbol classes $S^{-}[-\infty], \tilde{S}^{+}[\mu, \infty], \quad \tilde{S}^{-}[\mu, \kappa, \infty], \widetilde{S}^{-}[\mu, \infty]$ by $S^{-}[-\infty]=\cap S^{-}[\mu]$, $\tilde{S}^{+}[\mu, \infty]=\bigcap_{\kappa>0} \tilde{S}^{+}[\mu, \kappa], \quad \tilde{S}^{-}[\mu, \kappa, \infty]=\bigcap_{\lambda>0} \tilde{S}^{-}[\mu, \kappa, \lambda], \quad \tilde{S}^{-}[\mu, \infty]$ $=\bigcap_{\kappa>0} \widetilde{S}^{-}[\mu, \kappa]$.

As a final step of our preparation to describe our theorem, let us clarify the definition of parametrix.

Definition. Corresponding to each $i(1 \leq i \leq m)$, we call $E_{\imath}^{ \pm}(t, s)$ a parametrix if $E_{i}^{ \pm}(t, s) g \in C^{\infty}\left(\Delta_{ \pm} ; \mathscr{D}^{\prime}\left(\boldsymbol{R}^{n}\right)\right)$ for each $g \in \mathscr{E}^{\prime}\left(\boldsymbol{R}^{n}\right)$ and it satisfies

$$
\left\{\begin{array}{l}
P E_{i}^{ \pm} \equiv 0 \text { in } s<t, \\
\left.D_{t}^{h} E_{\imath}^{ \pm}\right|_{t=s} \equiv \delta_{h, i-1} I \quad(0 \leq h \leq m-1),
\end{array}\right.
$$

where $\Delta_{ \pm}=\left\{(t, s) \in\left[-T_{0}, T_{0}\right] \times\left[-T_{0}, T_{0}\right] ; s \leq t, \pm s, \pm t \geq 0\right\}$ and the symbol " $\equiv$ " stands for an equality modulo an integral operator with $C^{\infty}$ kernel. In the case, $s, t$ vary over $\Delta=\left\{(t, s) ;-T_{0} \leq s \leq t \leq T_{0}\right\}$, we also define parametrices $E_{i}(t, s)(1 \leq i \leq m)$ in the same way as we did for $E_{i}^{ \pm}(t, s)(1 \leq i \leq m)$. The only modification is to replace $\Delta_{ \pm}$by $\Delta$. If equalities hold in place of “ $\equiv$ ", then the above parametrices are called fundamental solutions.

Theorem 1. There exist $T_{0}>0$ and symbols

$$
\begin{aligned}
& a_{i, j}^{+}(t, x, \xi) \in \bigcap_{\varepsilon>0} \tilde{S}^{+}\left[(l+1)^{-1}\left(m_{j}^{+}-i+1+\varepsilon\right), m_{j}^{+}+\varepsilon\right], \\
& \tilde{a}_{i, j}^{+}(t, x, \xi) \in \bigcap_{\varepsilon>0} \tilde{S}^{+}\left[(l+1)^{-1}\left(m_{j}^{+}-i+1+\varepsilon\right), \infty\right](1 \leq i, j \leq m), \\
& a_{i, j}^{-}(t, s, x, \xi) \in \bigcap_{\varepsilon>0} \tilde{S}^{-}\left[2 \varepsilon(l+1)^{-1}-(i-1), m_{j}^{+}+\varepsilon, m_{j}^{-}-l(i-1)+\varepsilon\right], \\
& \tilde{a}_{i, j}^{-}(t, s, x, \xi) \in \bigcap_{\varepsilon>0} \tilde{S}^{-}\left[2 \varepsilon(l+1)^{-1}-(i-1), m_{-}^{j}+\varepsilon, \infty\right], \\
& \tilde{a}_{i, j}^{-}(t, s, x, \xi) \in \bigcap_{\varepsilon>0} \tilde{S}^{-}\left[2 \varepsilon(l+1)^{-1}-(i-1), \infty\right] \quad(1 \leq i, j \leq m)
\end{aligned}
$$


such that parametrices $E_{i}^{+}(t, 0)(1 \leq i \leq m)$ and $E_{i}^{-}(t, s)(1 \leq i \leq m)$ are given by

$$
\begin{aligned}
& \left(E_{i}^{+}(t, 0) \cdot\right)(x) \\
& =(2 \pi)^{-n} \sum_{j=1}^{m} O_{s}-\iint_{\times} \exp \left[\sqrt{-1}\left(\phi_{j}(t, 0, x, \xi)-y \cdot \xi\right)\right] \chi(\xi) \\
& \quad\left(0 \leq t \leq T_{i, j}^{+}(t, x, \xi)+\tilde{a}_{i, j}^{+}(t, x, \xi)\right) \cdot d y d \xi \\
& \quad\left(0 \mathbb{R}^{n}\right)^{*}
\end{aligned}
$$

(2. 12) $\left(E_{i}^{-}(t, s) \cdot\right)(x)$

$$
\begin{aligned}
= & (2 \pi)^{-n} \sum_{j=1}^{m} O_{s}-\iint \exp \left[\sqrt{-1}\left(\phi_{j}(t, s, x, \xi)-y \cdot \xi\right)\right] \chi(\xi) \\
& \times\left(a_{i, j}^{-}(t, s, x, \xi)+\tilde{a}_{i, j}^{-}(t, s, x, \xi)+\tilde{a}_{i, j}^{-}(t, s, x, \xi)\right) \cdot d y d \xi \\
& \quad\left(-T_{0} \leq s \leq t \leq 0, x \in \mathbb{R}^{n}\right),
\end{aligned}
$$

where $\chi(\xi) \in C_{0}^{\infty}\left(\mathbb{R}^{n}\right) ; \chi(\xi)=0\left(|\xi| \leq \frac{1}{2}\right), \quad \chi(\xi)=1 \quad(|\xi| \geq 1)$. In addition $\tilde{a}_{i, j}^{+}(1 \leq i, j \leq m)$ are flat at $t=0$ and $\tilde{a}_{i, j}^{-}(1 \leq j \leq m)$ are flat at $s=0$. Moreover,

(2.13) $\quad a_{i, j}^{+}(t, x, \xi)-T_{+}^{(i, j)}(x, \xi) V_{j}^{+}(t, x, \xi)$

$$
\in \bigcap_{\varepsilon>0} \tilde{S}^{+}\left[(l+1)^{-1}\left(m_{j}^{+}-i+1+\varepsilon\right), m_{j}^{+}+1+\varepsilon\right],
$$

(2.14) $\quad a_{i, j}^{-}(t, s, x, \xi)-\overline{a_{i, j, 0}^{-, 0}}(t, s, x, \xi)$

$$
\begin{aligned}
\in & \cap \tilde{S}_{\varepsilon>0}^{-}\left[2 \varepsilon(l+1)^{-1}-(i-1), m_{j}^{+}+1+\varepsilon, m_{j}^{-}-l(i-1)+\varepsilon\right] \\
& +\bigcap_{\varepsilon>0} \tilde{S}^{-}\left[2 \varepsilon(l+1)^{-1}-(i-1), m_{j}^{+}+\varepsilon, m_{j}^{-}-l(i-1)+1+\varepsilon\right],
\end{aligned}
$$

(2.15) $\quad a_{i, j, 0}^{-, 0}(t, s, x, \xi)$

$$
=\operatorname{det}\left(T_{-}^{(i, j)}(x, \xi)\right)_{1 \leq i, j \leq m} V_{j}^{-}(t, x, \xi) \widetilde{V}_{j, i-1}^{-}(s, x, \xi) .
$$

Remark. If we put

$$
\begin{aligned}
& \text { (2. 16) } U_{i}^{-}(t, s, x, \xi)=\sum_{j=1}^{m} \exp \left[\sqrt{-1}(l+1)^{-1}\left(t^{l+1}-s^{l+1}\right) \lambda_{j}(0, x, \xi)\right] \text {. } \\
& a_{i, j, 0}^{-, 0}(t, s, x, \xi) \text {, }
\end{aligned}
$$

then $U_{i}^{-}$satisfies

$$
L_{0} U_{i}^{-}=0,\left.D_{t}^{h} U_{i}^{-}\right|_{t=s}=\delta_{h, i-1}(0 \leq h \leq m-1) .
$$

Also $U_{i}^{-}$can be rewrit ten in the form:

$$
\begin{aligned}
x, & \xi)=\sum_{j=1}^{m} \sum_{\mu=1}^{m} \exp \left[-\sqrt{-1} s^{l+1}(l+1)^{-1} \lambda_{\mu}(0, x, \xi)\right] \\
& \times U_{j}(t, x, \xi) \widetilde{T}_{-}^{(j, \mu)}(x, \xi) \widetilde{V}_{\mu, i-1}^{-}(s, x, \xi) \\
= & \sum_{j=1}^{m} \exp \left[\sqrt{-1}(l+1)^{-1}\left(t^{l+1}-s^{l+1}\right) \lambda_{j}(0, x, \xi)\right] \\
& \times \operatorname{det}\left(T_{-}^{(i, j)}(x, \xi)\right)_{1 \leq i, j \leq m} \cdot V_{j}^{-}(t, x, \xi) \widetilde{V}_{j, i-1}(s, x, \xi) .
\end{aligned}
$$

* We have put " $O s$ " before integral signs to denote the usual oscillatory integral. 
This is proved in Lemma 3.5.

Now let $s<0$ be appropriately small. Since the parametrix $E_{i}(t, s)$ is given by $E_{i}(t, s) \equiv E_{i}^{-}(t, s)$ for $t \leq 0$ and $E_{i}(t, s) \equiv \sum_{k=1}^{m} E_{k}^{+}(t, 0) \cdot$ $\left.\left(D_{t}^{k-1} E_{i}^{-}(t, s)\right)\right|_{t=0}$ if $t>0$, the composition formula for Fourier integral operators and Theorem 1 imply the following theorem.

\section{Theorem 2}

(2.19) $\quad\left(E_{i}(t, s) \cdot\right)(x)$

$$
\begin{gathered}
=(2 \pi)^{-n} \sum_{\nu, \mu=1}^{m} O_{s}-\iint \exp \left[\sqrt{-1}\left(\phi_{\nu, \mu}(t, s, x, \eta)-y \cdot \eta\right)\right] \\
a_{i, \nu, \mu}(t, s, x, \eta) \cdot d y d \eta
\end{gathered}
$$

for $t>0$. Here, if $s, t$ are small enough, there exists $R>0$ such that (2.20) the main part of $a_{i, \nu, \mu}(t, s, x, \eta)$

$$
\begin{aligned}
= & \sum_{j=1}^{m} T_{+}^{(j, \nu)}\left(x, \nabla_{x} \phi_{\nu, \mu}(t, s, x, \eta)\right) \widetilde{T}^{(j, \mu)}\left(\nabla_{\eta} \phi_{\nu, \mu}(t, s, x, \eta), \eta\right) \\
& \times V_{\nu}^{+}\left(t, x, \nabla_{x} \phi_{\nu, \mu}(t, s, x, \eta)\right) \tilde{V}_{\mu, i-1}\left(s, \nabla_{\eta} \phi_{\nu, \mu}(t, s, x, \eta), \eta\right) \\
& (\text { nonzero factor }) \quad \text { for }|\eta| \geq R .
\end{aligned}
$$

(2) Let $i(0 \leq i \leq m-1)$ be an integer and $u_{h} \in \mathscr{E}^{\prime}\left(R^{n}\right)(0 \leq h \leq m-1)$ whose wave front sets $W F\left(u_{h}\right)(0 \leq h \leq m-1)$ satisfy $\cup W F\left(u_{h}\right)=\phi$, $W F\left(u_{i}\right)=\left\{\left(y^{0}, \rho \eta^{0}\right) ; \rho>0\right\}$. Let $u(t, s, x)$ be a solution of the Cauchy problem: $P u=0,\left.\quad D_{t}^{h} u\right|_{t=s}=u_{h}(0 \leq h \leq m-1)$. Suppose the following condition (\#) holds for a particular pair $\left(\nu_{0}, \mu_{0}\right)$ and a sufficiently small $s^{\prime}, t^{\prime}\left(s \leq s^{\prime}<0<t^{\prime}\right)$

(\#) $\sum_{j=1}^{m} T_{+}^{\left(j, \nu_{0}\right)}\left(T_{a_{0^{\prime}} \mu_{0}}\left(t^{\prime}, s^{\prime}\right) \circ T_{\mu_{0}}\left(s^{\prime}, s\right)\left(y^{0}, \eta^{0}\right)\right) \widetilde{T}_{-}^{\left(j, \mu_{0^{\prime}}\right)}\left(T_{\mu_{0}}\left(s^{\prime}, s\right)\right)\left(y^{0}, \eta^{0}\right) \neq 0$. Then, for any $t\left(0<t \leq T_{0}\right)$, the wave front set $W F(u(t, s))$ of $u(t, s)$ contains $T_{\nu_{0}, \mu_{0}}(t, s)\left(y^{0}, \eta^{0}\right)$.

Remark. (1) Since $T_{\mu_{0}}(0,0)(y, \eta)=T_{\nu_{0}, \mu_{0}}(0,0)(y, \eta)=(y, \eta)$, the following condition (\#) $)^{\prime}$ which is similar to that of Amano [2] implies (\#) if $T_{0}$ is sufficiently small.

$$
\sum_{j=1}^{m} T_{+}^{\left(j, \nu_{0}\right)}\left(y^{0}, \eta^{0}\right) \widetilde{T}_{-}^{\left(j, \mu_{0}\right)}\left(y^{0}, \eta^{0}\right) \neq 0 .
$$

The left-hand side of $(\#)^{\prime}$ is the so-called Stokes' multiplier.

(2) As we mentioned in Section 1, we can easily derive many other conditions instead of (\#) by observing the proof of Theorem 1 and 2 .

For $m=2, \quad T_{ \pm}^{(i, j)}=T_{ \pm}^{(i, j)}(x, \xi)(i, j=1,2)$ can be given explicitly. Namely we have the following theorem. 
Theorem 3. Let $m=2, \alpha_{i}=\alpha_{i}(x, \xi)=\sqrt{-1} \lambda_{i}(0, x, \xi /|\xi|), \kappa_{i}=\kappa_{i}(x$, $\xi)=-\mu_{i}(x, \xi)(l+1)^{-1}, \quad \gamma_{i}=\gamma_{i}(x, \xi)=\kappa_{i}-1(i=1,2)$ and $\delta_{2}=(l+1)^{-1}$. Assume $\kappa_{i}, \kappa_{i}+\delta_{2} \notin \boldsymbol{Z}(i=1,2)$.

(1. i) When $\lambda_{1}(0, x, \xi)>\lambda_{2}(0, x, \xi)$,

$$
\left\{\begin{aligned}
T_{+}^{(1.1)} & =(l+1)^{\kappa_{1}} \Gamma\left(\delta_{2}\right)^{-1} \Gamma\left(\gamma_{1}+\delta_{2}+1\right)\left(\alpha_{2}-\alpha_{1}\right)^{\gamma_{2}+\delta_{2}} \\
& \times\left(1-\exp \left[-\pi \sqrt{-1}\left(\gamma_{1}+\delta_{2}\right)\right] \sin \pi\left(\gamma_{2}+\delta_{2}\right) / \sin \pi\left(\gamma_{1}+\gamma_{2}+2 \delta_{2}\right)\right), \\
T_{+}^{(1.2)} & =(l+1)^{\kappa_{2}} \Gamma\left(\delta_{2}\right)^{-1} \Gamma\left(\gamma_{2}+\delta_{2}+1\right)\left(\alpha_{1}-\alpha_{2}\right)^{\gamma_{1}+\delta_{2}} \\
& \times \exp \left[-\pi \sqrt{-1}\left(\gamma_{1}+\delta_{2}\right)\right] \sin \pi\left(\gamma_{2}+\delta_{2}\right) / \sin \pi\left(\gamma_{1}+\gamma_{2}+2 \delta_{2}\right), \\
T_{+}^{(2,1)} & =\sqrt{-1}|\xi|^{-\delta_{2}}(l+1)^{\kappa_{1}+\delta_{2}} \Gamma\left(-\delta_{2}\right)^{-1} \Gamma\left(\gamma_{1}+1\right)\left(\alpha_{2}-\alpha_{1}\right)^{\gamma_{2}} \\
& \times\left(1-\exp \left[-\pi \sqrt{-1} \gamma_{1}\right] \sin \pi \gamma_{2} / \sin \pi\left(r_{1}+\gamma_{2}\right)\right), \\
T_{+}^{(2,2)} & =\sqrt{-1}|\xi|^{-\delta_{2}}(l+1)^{\kappa_{2}+\delta_{2}} \Gamma\left(-\delta_{2}\right)^{-1} \Gamma\left(\gamma_{2}+1\right)\left(\alpha_{1}-\alpha_{2}\right)^{\gamma_{1}} \\
& \times \exp \left[-\pi \sqrt{-1} \gamma_{1}\right] \sin \pi \gamma_{2} / \sin \pi\left(\gamma_{1}+\gamma_{2}\right)
\end{aligned}\right.
$$

(1. ii) When $\lambda_{2}(0, x, \xi)>\lambda_{1}(0, x, \xi)$,

$$
\left\{\begin{aligned}
T_{+}^{(1.1)} & =(l+1)^{\kappa_{1}} \Gamma\left(\delta_{2}\right)^{-1} \Gamma\left(\gamma_{1}+\delta_{2}+1\right)\left(\alpha_{2}-\alpha_{1}\right)^{\gamma_{2}+\delta_{2}} \\
& \times \exp \left[-\pi \sqrt{-1}\left(\gamma_{2}+\delta_{2}\right)\right] \sin \pi\left(\gamma_{1}+\delta_{2}\right) / \sin \pi\left(\gamma_{1}+\gamma_{2}+2 \delta_{2}\right), \\
T_{+}^{(1,2)} & =(l+1)^{\kappa_{2}} \Gamma\left(\delta_{2}\right)^{-1} \Gamma\left(\gamma_{2}+\delta_{2}+1\right)\left(\alpha_{1}-\alpha_{2}\right)^{\gamma_{1}+\delta_{2}} \\
& \times\left(1-\exp \left[-\pi \sqrt{-1}\left(\gamma_{2}+\delta_{2}\right)\right] \sin \pi\left(\gamma_{1}+\delta_{2}\right) / \sin \pi\left(\gamma_{1}+\gamma_{2}+2 \delta_{2}\right)\right), \\
T_{+}^{(2,1)} & =\sqrt{-1}|\xi|^{-\delta_{2}}(l+1)^{\kappa_{1}+\delta_{2}} \Gamma\left(-\delta_{2}\right)^{-1} \Gamma\left(\gamma_{1}+1\right)\left(\alpha_{2}-\alpha_{1}\right)^{\gamma_{2}} \\
& \times \exp \left[-\pi \sqrt{-1} \gamma_{2}\right] \sin \pi \gamma_{1} / \sin \pi\left(\gamma_{1}+\gamma_{2}\right), \\
T_{+}^{(2,2)} & =\sqrt{-1}|\xi|^{-\delta_{2}}(l+1)^{\kappa_{2}+\delta_{2}} \Gamma\left(-\delta_{2}\right)^{-1} \Gamma\left(\gamma_{2}+1\right)\left(\alpha_{1}-\alpha_{2}\right)^{\gamma_{1}} \\
& \times\left(1-\exp \left[-\pi \sqrt{-1} \gamma_{2}\right] \sin \pi \gamma_{1} / \sin \pi\left(\gamma_{1}+\gamma_{2}\right)\right)
\end{aligned}\right.
$$

(2) In the case $l$ is odd, we have

$$
\left\{\begin{array}{l}
T_{-}^{(1, j)}=(-1)^{\mu_{j}(x, \xi)} T_{+}^{(1, j)}(x, \xi) \\
T_{-}^{(2, j)}=(-1)^{\mu_{j}(x, \xi)+1} T_{+}^{(2, j)}(x, \xi)
\end{array} \quad(j=1,2)\right.
$$

(3) In the case $l$ is even, we have the following two cases.

(3. i) When $\lambda_{1}(0, x, \xi)>\lambda_{2}(0, x, \xi)$,

$$
\left\{\begin{aligned}
T_{-}^{(1,1)} & =(-1)^{\mu_{1}}(l+1)^{\kappa_{1}} \Gamma\left(\delta_{2}\right)^{-1} \Gamma\left(\gamma_{1}+\delta_{2}+1\right)\left(\alpha_{1}-\alpha_{2}\right)^{\gamma_{2}+\delta_{2}} \\
& \times \exp \left[-\pi \sqrt{-1}\left(\gamma_{2}+\delta_{2}\right)\right] \sin \pi\left(\gamma_{1}+\delta_{2}\right) / \sin \pi\left(\gamma_{1}+\gamma_{2}+2 \delta_{2}\right) \\
T_{-}^{(1,2)} & =(-1)^{\mu_{2}}(l+1)^{\kappa_{2}} \Gamma\left(\delta_{2}\right)^{-1} \Gamma\left(\gamma_{2}+\delta_{2}+1\right)\left(\alpha_{2}-\alpha_{1}\right)^{\gamma_{1}+\delta_{2}} \\
& \times\left(1-\exp \left[-\pi \sqrt{-1}\left(\gamma_{2}+\delta_{2}\right)\right] \sin \pi\left(\gamma_{1}+\delta_{2}\right) / \sin \pi\left(\gamma_{1}+\gamma_{2}+2 \delta_{2}\right)\right) \\
T_{-}^{(2,1)} & =\sqrt{-1}|\xi|^{-\delta_{2}}(-1)^{\mu_{1}+1}(l+1)^{\kappa_{1}+\delta_{2}} \Gamma\left(-\delta_{2}\right)^{-1} \Gamma\left(\gamma_{1}+1\right)\left(\alpha_{1}-\alpha_{2}\right)^{\gamma_{2}} \\
& \times \exp \left[-\pi \sqrt{-1} \gamma_{2}\right] \sin \pi \gamma_{1} / \sin \pi\left(\gamma_{1}+\gamma_{2}\right), \\
T_{-}^{(2,2)} & =\sqrt{-1}|\xi|^{-\delta_{2}}(-1)^{\mu_{2}+1}(l+1)^{\kappa_{2}+\delta_{2}} \Gamma\left(-\delta_{2}\right)^{-1} \Gamma\left(\gamma_{2}+1\right)\left(a^{1}-\alpha_{2}\right)^{\gamma_{1}} \\
& \times\left(1-\exp \left[-\pi \sqrt{-1} \gamma_{2}\right] \sin \pi \gamma_{1} / \sin \pi\left(\gamma_{1}+\gamma_{2}\right)\right) .
\end{aligned}\right.
$$


(3. ii) When $\lambda_{2}(0, x, \xi)>\lambda_{1}(0, x, \xi)$,

$$
\left\{\begin{aligned}
T_{-}^{(1,1)} & =(-1)^{\mu_{1}}(l+1)^{\kappa_{1}} \Gamma\left(\delta_{2}\right)^{-1} \Gamma\left(\gamma_{1}+\delta_{2}+1\right)\left(\alpha_{1}-\alpha_{2}\right)^{\gamma_{2}+\delta_{2}} \\
& \times\left(1-\exp \left[-\pi \sqrt{-1}\left(\gamma_{1}+\delta_{2}\right)\right] \sin \pi\left(\gamma_{2}+\delta_{2}\right) / \sin \pi\left(\gamma_{1}+\gamma_{2}+2 \delta_{2}\right)\right), \\
T_{-}^{(1,2)} & =(-1)^{\mu_{2}}(l+1)^{\kappa_{2}} \Gamma\left(\delta_{2}\right)^{-1} \Gamma\left(\gamma_{2}+\delta_{2}+1\right)\left(\alpha_{2}-\alpha_{1}\right)^{\gamma_{1}+\delta_{2}} \\
& \times \exp \left[-\pi \sqrt{-1}\left(\gamma_{1}+\delta_{2}\right)\right] \sin \pi\left(\gamma_{2}+\delta_{2}\right) / \sin \pi\left(\gamma_{1}+\gamma_{2}+2 \delta_{2}\right), \\
T_{-}^{(2,1)} & =\sqrt{-1}|\xi|^{-\delta_{2}}(-1)^{\mu_{1}+1}(l+1)^{\kappa_{1}+\delta_{2}} \Gamma\left(-\delta_{2}\right)^{-1} \Gamma\left(\gamma_{1}+1\right)\left(\alpha_{1}-\alpha_{2}\right)^{\gamma_{2}} \\
& \times\left(1-\exp \left[-\pi \sqrt{-1} \gamma_{1}\right] \sin \pi \gamma_{2} / \sin \pi\left(\gamma_{1}+\gamma_{2}\right)\right), \\
T_{-}^{(2,2)} & =\sqrt{-1}|\xi|^{-\delta_{2}}(-1)^{\mu_{2}+1}(l+1)^{\kappa_{2}+\delta_{2}} \Gamma\left(-\delta_{2}\right)^{-1} \Gamma\left(\gamma_{2}+1\right)\left(\alpha_{2}-\alpha_{1}\right)^{\gamma_{1}} \\
& \times \exp \left[-\pi \sqrt{-1} \gamma_{1}\right] \sin \pi \gamma_{2} / \sin \pi\left(\gamma_{1}+\gamma_{2}\right) .
\end{aligned}\right.
$$

Here $\Gamma(\sigma)$ denotes the gamma function.

Remark. Since $T_{ \pm}^{(i, j)}(x, \xi)(1 \leq i, j \leq 2)$ may have poles for certain values of $\kappa_{i}$ and $\kappa_{i}+\delta_{2}$, we have put the additional conditions $\kappa_{i}$, $\kappa_{1}+\delta_{2} \notin \boldsymbol{Z}$ in Theorem 3. However, the condition (\#)' has a meaning even for an exceptional value of $\kappa_{i}$ or $\kappa_{i}+\delta_{2}$ as far as the Stokes' multiplier is well defined. In Section 5 we will show this certainly happens for the Taniguchi and Tozaki's operator.

\section{§ 3. Construction of Parametrix $E_{m}^{-}(t, s)$}

We owe the basic idea of our construction to that of Yoshikawa [27]. However, we cannot apply his method directly to ours. The reason why is that Proposition 3.4 of Yoshikawa [27] is not valid any more in the case $|s| \geq|t|$ and this is the case we have for $s, t \in \Delta_{-}$. To avoid this difficulty, we adopt the following lemma due to Shinkai*.

Lemma 3.1. Parametrix $E_{m}^{-}(t, s)$ is also obtained by solving the Cauchy problem:

$$
\left\{\begin{array}{l}
Q E_{m}^{-} \equiv 0, \\
\left.D_{s}^{h} E_{m}^{-}\right|_{s=t} \equiv \delta_{h, m-1}(-1)^{m-1} I(0 \leq h \leq m-1),
\end{array}\right.
$$

where $Q=\sum_{j=0}^{m} \sum_{i=0}^{m-j}\left(-D_{s}\right)^{m-j-i}\left(P_{i, j}\left(s, x, D_{x}\right)\right)$.

Proof. Introduce $U={ }^{t}\left[u, D_{t} u, \cdots, D_{t}^{m-1} u\right]^{* *}$ and rewrite $P u=0$

* We noticed this by an oral communication with him.

** The symbol " $t$ " denotes the transpose of a vector and a matrix. 
into a system $D_{t} U-A(t) U=0$. Let $K(s, t)$ be a fundamental solution of the Cauchy problem $\left({ }^{*}\right) D_{s} K=-A^{*}(s) K, K(t, t)=I$. Then according to Kumanogo [11], $K(s, t)$ is unique and satisfies the semigroup property: $K(s, \sigma) \cdot K(\sigma, t)=K(s, t)(s \leq \sigma \leq t \leq 0)$. Suppose $F(t, s)$ $(s \leq t \leq 0)$ satisfies

$$
D_{s} F=-F A(s), F(t, t)=I,
$$

the adjoint $F^{*}(t, s)$ of $F(t, s)$ satisfies $D_{s} F^{*}=-A^{*}(s) F^{*}, F^{*}(t, t)=I$. Then the uniqueness and the semigroup property of the fundamental solution $K(s, t)$ imply $F^{*}(t, s)=K(s, t)$ and $F(t, \sigma) \cdot F(\sigma, s)=F(t, s)$ $(s \leq \sigma \leq t \leq 0)$. So if we differentiate the latter equality by $\sigma$, we obtain $D_{\sigma} F(t, \sigma) \cdot F(\sigma, s)+F(t, \sigma) D_{\sigma} F(\sigma, s)=0$. Here, if we remind $D_{\sigma} F(t, \sigma)=-F(t, \sigma) A(\sigma)$, we have $D_{t} F(t, s)-A(t) F(t, s)=0$. Thus, the $(1, m)$-th component of $F(t, s)$ is the desired fundamental solution $E_{m}^{-}(t, s)$. Then writing down the condition for $E_{m}^{-}(t, s)$ from (3.2), we obtain (3.1).

Q. E. D.

Remark. Since the definition of Kumanogo's fundamental solution is different from ours, there is a leap in our argument. To fill the leap, we have to construct a fundamental solution for the Cauchy problem $\left(^{*}\right)$ and estimate it as an operator on the space $H^{-\infty}\left(\mathbb{R}^{n}\right)$ which is all the union of Sobolev spaces so that we can consider our fundamental solution as that of Kumanogo [11]. This can be done by using an argument analogous to that of Yoshikawa [27]. However, we are only interested in the propagation of singularities, we avoid the redundant arguments.

Let's construct our parametrix so as to satisfy (3.1). Although the outline of our construction is basically the same as that of [27], it is much more complicated. We seek $E_{m}^{-}(t, s)$ in the form $(2.12)$. In (2.12), $\tilde{a}_{m, j}^{-}, \tilde{a}_{m, j}^{-}(1 \leq j \leq m)$ are compensating factors which compensate the errors caused by determining $a_{m, j}^{-}(1 \leq j \leq m)$. So let's restrict our attention to determining $a_{m, j}^{-}(1 \leq j \leq m)$ appropriately for a whil..

Although, strictly speaking, the following arguments need some modification for $|\xi| \leq 1$, we neglect the factor $x(\xi)$ for simplicity.

Set

$$
\begin{aligned}
\left(F_{j}(t, s) \cdot\right)(x)= & (2 \pi)^{-n} O_{s^{-}} \iint \exp \left[\sqrt{-1}\left(\phi_{j}(t, s, x, \xi)-y \cdot \xi\right)\right] \\
& \times a(t, s, x, \xi) \cdot(y) d y d \xi
\end{aligned}
$$


and define the so-called transport operator $T_{j}$ by

$$
\begin{aligned}
\left(\left(Q F_{j}\right)(t, s) \cdot\right)(x)= & (2 \pi)^{-n} O_{s}-\iint \exp \left[\sqrt{-1}\left(\phi_{j}(t, s, x, \xi)-y \cdot \xi\right)\right] \\
& \times\left(T_{j} a\right)(t, s, x, \xi) \cdot(y) d y d \xi .
\end{aligned}
$$

For further arguments, we prepare the following definition.

Definition. An operator $r\left(t, s, x, \xi, D_{t}, D_{s}, D_{x}, D_{\xi}\right)$ is called semihomogeneous (respectively, homogeneous) of degree $\sigma$, if it satisfies

$$
\begin{aligned}
& \quad r\left(\rho^{-\frac{1}{l+1}} t, \rho^{-\frac{1}{l+1}} s, x, \rho \xi, \rho^{\frac{1}{l+1}} D_{t}, \rho^{\frac{1}{l+1}} D_{s}, D_{x}, \rho^{-1} D_{\xi}\right) \\
&= \rho^{\sigma} r\left(t, s, x, \xi, D_{t}, D_{s}, D_{x}, D_{\xi}\right) \\
& \text { (respectively, } r\left(t, s, x, \rho \xi, D_{t}, D_{s}, D_{x}, \rho^{-1} D_{\xi}\right) \\
&\left.=\rho^{\sigma} r\left(t, s, x, \xi, D_{t}, D_{s}, D_{x}, D_{\xi}\right)\right) \quad \text { for } \rho>0 .
\end{aligned}
$$

Concerning the decomposition of $T_{j}$ into semi-homogeneous parts, we have the following lemma.

\section{Lemma 3.2.}

$$
T_{j} \approx \sum_{k=0}^{\infty} T_{j, k}
$$

where $T_{j, k}=\sum_{q=0}^{k} t^{q} T_{j, k, q}$ and $T_{j, k, q}$ is semi-homogeneous of degree ( $m-k$ $+q) /(l+1)$ and independent of $t$. Furthermore, the number of $s$ included in each term of $T_{j, k, q}$ is at most $l(m-1)+k-q-1+$ (the power of $D_{s}$ included in this term) and $T_{j, 0} \equiv T_{j, 0,0}$ is given by

$$
\begin{aligned}
\widetilde{T}_{j, 0}= & \exp \left[\sqrt{-1} \frac{s^{l+1}}{l+1} \lambda_{j}(0, x, \xi)\right] \\
& \times M_{0}\left(\exp \left[-\sqrt{-1} \frac{s^{l+1}}{l+1} \lambda_{j}(0, x, \xi)\right] \cdot\right)
\end{aligned}
$$

with

$$
M_{0}=\sum_{i l \geq j, m-j-i \geq 0}\left(-D_{s}\right)^{m-j-i}\left(s^{i l-j} \tilde{P}_{i, j}(0, x, \xi) \cdot\right) .
$$

Here the meaning of the symbol " $\approx$ " is as follows. For each nonnegative integer $N$, define $\hat{T}_{j, N+1, q}$ by

$$
T_{j}=\sum_{k=0}^{N} \sum_{q=0}^{k} t^{q} T_{j, k, q}+\sum_{q=0}^{N+1} t^{q} \hat{T}_{j, N+1, q}
$$

then the effect of applying $\hat{T}_{j, N+1, q}$ to a symbol $\in \tilde{S}^{-}[\mu, \kappa, \lambda]$ is at most multiplying an element of $\tilde{S}^{-}[m-1,0, l(m-1)+N-q]$. 
Proof. The outline of the proof is as follows. Expand $T_{j}$ using the composition formula of Fourier integral operator and pseudodifferential operator and then expand each term of this expansion with respect to $t$ and $s$ into Taylor series around $t=s=0$.

Let's illustrate this in details. In the rest of the proof, we drop the index $j$ and represent $T_{j}, T_{j, k, q}, a_{j}, \phi_{j}$ by $T, T_{k, q}, a, \phi$. The composition formula of Fourier integral operator and pseudo-differential operator entails

$$
\begin{aligned}
& (T a)(t, s, x, \xi) \\
& \quad \sim \sum_{\alpha} \frac{1}{\alpha !} \sum_{1}(-1)^{m-j-1}\left(\begin{array}{c}
m-j-i \\
k
\end{array}\right)\left(\begin{array}{l}
\alpha \\
\beta
\end{array}\right) D_{s}^{m-j-i-k}\left\{\partial_{\xi}^{\alpha-\beta} a(t, s, x, \xi)\right. \\
& \left.\quad \times \Psi_{\gamma, \beta}(t, s, x, \xi) P_{i, j_{(\alpha+\gamma)}}\left(s, \nabla_{\xi} \phi(t, s, x, \xi), \xi\right)\right\} \Phi_{\nu, k}(t, s, x, \xi),
\end{aligned}
$$

where the symbol " " stands for the usual asymptotic equality for symbols and $\sum_{1}$ is a summation with respect to the non-negative integers $j, i, k, \nu, \beta, \gamma(0 \leq j \leq m, 0 \leq i \leq m-j, 0 \leq k \leq m-j-1,0 \leq \nu \leq k$, $\beta \leq \alpha, 0 \leq|\gamma| \leq|\beta|)$ and $P_{i, j}(\alpha+\gamma), \Phi_{\nu, k}, \Psi_{\gamma, \beta}$ are given by

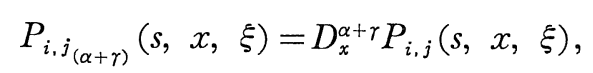

$$
\begin{aligned}
& \Phi_{\nu, k}(t, s, x, \xi)=\left\{\begin{array}{lr}
1 & (\nu=k=0), \\
0 & (\nu=0, k \neq 0), \\
(-1)^{k}(\sqrt{-1})^{k+\nu} \sum_{2} k !(\nu !)^{-1} & \\
\prod_{\mu=1}^{\nu}\left(k_{\mu} !\right)^{-1} \partial_{s}^{k} \phi(t, s, x, \xi) & (1 \leq \nu \leq k)
\end{array}\right. \\
& \Psi_{r, \beta}(t, s, x, \xi)=\left\{\begin{array}{rr}
1 & (\beta=\gamma=0), \\
0 & (\gamma=0, \beta \neq 0), \\
\sum_{3} \beta !\left(\gamma ! \beta^{1,1} ! \cdots \beta^{1, \gamma_{1}} ! \cdots \beta^{n, \gamma_{n}} !\right)^{-1} \bullet & \\
\prod_{q=1}^{n} \prod_{r=1}^{\gamma_{q}}\left(\left|\beta^{q, r}\right|+1\right)^{-1} \partial_{\xi}^{\beta_{\xi}^{q, r}} \partial_{\xi_{q}} \phi(t, s, x, \xi) & (1 \leq|\gamma| \leq|\beta|) .
\end{array}\right.
\end{aligned}
$$

Here, the summation $\Sigma_{2}$ is taken with respect to non-negative integers $k_{1}, \cdots, \quad k_{\nu}\left(k_{1}+\cdots+k_{\nu}=k, \quad k_{\mu} \neq 0\right)$ and $\sum_{3}$ is taken with respect to multi-indices $\gamma=\left(\gamma_{1}, \cdots, \gamma_{n}\right), \quad \beta^{q, r}\left(1 \leq q \leq n, 1 \leq r \leq \gamma_{q}\right)$ such that $\beta^{1,1}+\cdots+\beta^{1, \gamma_{1}}+\cdots+\beta^{n, 1}+\cdots+\beta^{n, \gamma_{n}}=\beta, \beta^{q, r} \neq 0$. Now let $a$ be semihomogeneous of degree zero and pick up the semi-homogeneous part of maximal degree from the right-hand side of (3.9). If we expand each $D_{s}^{m-j-i-k}\left\{\partial_{\xi}^{\alpha-\beta} a \cdot \Psi_{\gamma, \beta} \cdot P_{i, j_{(\alpha+\gamma)}}\right\} \Phi_{\nu, k}$ into Taylor series with respect to $s, t$ around $s=t=0$, then this yields terms whose degree of semi- 
homogeneity is at most $m(l+1)^{-1}-(|\alpha|+|\gamma|)$ for $i l \geq j, m-j-i \geq 0$ and $(m+i l-j)(l+1)^{-1}-(|\alpha|+|\gamma|)$ for $i l<j, m-j-i \geq 0$. Thus the semi-homogeneous part of maximal degree yields from

$$
\begin{gathered}
\sum_{4}(-1)^{m-j-i}\left(\begin{array}{c}
m-j-i \\
k
\end{array}\right) D_{s}^{m-j-i-k}\left\{a(t, s, x, \xi) P_{i, j}(s, x, \xi)\right\} \\
\Phi_{\nu, k}(t, s, x, \xi),
\end{gathered}
$$

where the summation $\sum_{4}$ is taken with respect to non-negative integers $i, j, k, \nu(i l \geq j, m-j-i \geq 0,0 \leq k \leq m-j-i, 0 \leq \nu \leq k)$.

Since $\phi(t, s, x, \xi)=x \cdot \xi+(l+1)^{-1} \lambda(0, x, \xi)\left(t^{l+1}-s^{l+1}\right)$

+ (the semi-homogeneous part of higher degree)

and assumption (A. 3), the semi-homogeneous part of maximal degree is

$$
\begin{gathered}
\sum_{4}(-1)^{m-j-i}\left(\begin{array}{c}
m-j-i \\
k
\end{array}\right) D_{s}^{m-j-i-k}\left\{a(t, s, x, \xi) s^{i l-j} \tilde{P}_{i, j}(0, x, \xi)\right\} \\
\Phi_{\nu, k}^{*}(s, x, \xi),
\end{gathered}
$$

where

$$
(3.12)^{\prime} \Phi_{\nu, k}^{*}(s, x, \xi)=\left\{\begin{array}{lr}
1 & (\nu=k=0), \\
0 & (\nu=0, k \neq 0), \\
(-1)^{k}(\sqrt{-1})^{k+\nu} \sum_{2} k !(\nu !)^{-1} & \\
\prod_{\mu=1}^{\nu}\left(k_{\mu} !\right)^{-1} \partial_{s}^{k}\left(-s^{l+1}(l+1)^{-1} \lambda(0, x,\right. & \xi)) \\
& (1 \leq \nu \leq k)
\end{array}\right.
$$

Observing the calculation which entailed (3.9), we easily obtain (3.6).

Next we proceed to prove the second assertion. Let $b\left(x, \xi, D_{\xi}\right) s^{\sigma} D_{s}^{\mu}$ be a general term of $T_{k, q}$, where $b\left(x, \xi, D_{\xi}\right)$ is homogeneous of degree $\delta$. We need to estimate $\delta$. Let's do this first. It is easy to see each of

$$
\Phi_{\nu, k} D_{s}^{m-j-i-k}\left\{\Psi_{r, \beta} P_{i, j(\alpha+\gamma)} \partial_{\xi}^{\alpha-\beta} \cdot\right\}
$$

is homogeneous of degree $\nu+i-|\alpha|$. Since $0 \leq k \leq m-j-i, 0 \leq \nu \leq k$, $\beta \leq \alpha, 0 \leq|\gamma| \leq|\beta|$, this becomes maximal when $j=0, \nu=k=m-i$, $|\alpha|=|\beta|=|\gamma|=0$. Thus the homogeneous part of maximal degree is

$$
\sum_{i=0}^{m}(-1)^{m-i} P_{i, 0}\left(s, \nabla_{\xi} \phi, \xi\right) \Phi_{m-i, m-i} .
$$

Taking account of $\Phi_{m-i, m-i}(t, s, x, \xi)=\left(\partial_{s} \phi(t, s, x, \xi)\right)^{m-i}(0 \leq i \leq m)$, this is equal to $P_{m}\left(s, \nabla_{\xi} \phi(t, s, x, \xi),-\partial_{s} \phi(t, s, x, \xi), \xi\right)=0$. Since the general terms of $T_{k, q}$ yield from these (3.13), we have obtained an estimate $\delta \leq m-1$. Using this fact, we can easily prove the second 
assertion of Lemma 3.2. Since $T_{k, q}$ is semi-homogeneous of degree $(m-k-q)(l+1)^{-1}$, we have $(-\sigma+\mu)(l+1)^{-1}+\delta=(m-k+q)(l+1)^{-1}$. Thus $\sigma-\mu=-m+k-q+\delta(l+1)$. Hence, we have $\sigma-\mu \leq l(m-1)$ $+k-q-1$ from $\delta \leq m-1$ and this completes the proof of the second assertion.

Finally, we prove the last assertion. Reminding the remainder formula of Taylor's expansion, it is easy to see each term of $\hat{T}_{N+1, q}$ has the form:

$$
c\left(t, s, x, \xi, D_{\xi}\right) s^{\sigma} D_{s}^{\mu}
$$

where $\sigma \leq l(m-1)+N-q+\mu$ and $c\left(t, s, x, \xi, D_{\xi}\right)$ sends $\tilde{S}^{-}[\gamma, \kappa, \lambda]$ into $\tilde{S}^{-}[\gamma+m-1, \kappa, \lambda]$. This immediately leads us to the last assertion if we note $S^{-}\left[\gamma^{\prime}\right] \cdot \tilde{S}^{-}[\gamma, \kappa, \lambda] \subset \tilde{S}^{-}\left[\gamma+\gamma^{\prime}, \kappa, \lambda\right]$ and $s^{\sigma} D_{s}^{\mu} \tilde{S}^{-}[\gamma, \kappa, \lambda]$ $\subset \tilde{S}^{-}[\gamma, \kappa, \lambda+\sigma-\mu]^{*}$.

Q. E. D.

A proof similar to Lemma 3. 2 leads to the following result.

\section{Lemma 3.3. Let}

$$
S_{j, h} \cdot=\left.\exp \left[-\sqrt{-1} \phi_{j}(t, s, x, \xi)\right] D_{s}^{h}\left(\exp \left[\sqrt{-1} \phi_{j}(t, s, x, \xi)\right] \cdot\right)\right|_{s=l} \cdot
$$

Then we have

$$
S_{j, h} \approx \sum_{k=0}^{\infty} S_{j, k, k}
$$

where $S_{j, h, k}$ is semi-homogeneous of degree $\frac{h-k}{l+1}$. Furthermore, the number of $t$ included in each term of $S_{j, h, k}$ is at most $h l+k+($ the power of $\left.D_{s}\right|_{s=t}$ included in this term) and $S_{j, h, 0}$ is given by

$$
\begin{aligned}
S_{j, h, 0^{\bullet}}= & \exp \left[\sqrt{-1} s^{l+1}(l+1)^{-1} \lambda_{j}(0, x, \xi)\right. \\
& \left.D_{s}^{h}\left(\exp \left[-\sqrt{-1} s^{l+1}(l+1)^{-1} \lambda_{j}(0, x, \xi)\right] \cdot\right)\right|_{s=t} .
\end{aligned}
$$

Here the meaning of the symbol " $\approx "$ is as follows. For each nonnegative integer $N$, define $\tilde{S}_{j, h+1}$ by

$$
S_{j, h}=\sum_{k=0}^{N} S_{j, h, k}+\hat{S}_{j, h, N+1}
$$

then $S_{j, h, N+1}$ sends $\tilde{S}^{-}[\mu, \kappa, \lambda]$ into $\tilde{S}^{-}[\mu+h, \kappa+\lambda+h l+N+1]$.

In the meanwhile, we argue formally. Consider the asymptotic equalities (3.5) and (3.14) as equalities. Also we assume that $a_{m, j}^{-}$ admits a formal expansion

\footnotetext{
* The left-hand side is the image of $s^{o} D_{s}^{\mu}$ applied to $\tilde{S}^{-}[\gamma, \kappa, \lambda]$.
} 


$$
a_{m, j}^{-}=\sum_{\nu=0}^{\infty} \sum_{\mu=0}^{\infty} a_{m, j, \nu}^{-, \mu}
$$

where each $a_{m, j, \nu}^{-, \mu}$ is semi-homogeneous of degree $-(\nu+\mu+m-1)(l$ $+1)^{-1}$. Expand $T_{j} a_{m, j}^{-}$and $S_{j, h} a_{m, j}^{-}$formally and assemble the terms which have the same degree of homogeneity. Then, as a sufficient condition for

$$
\left\{\begin{array}{l}
T_{j} a_{m, j}^{-}=0, \\
\sum_{j=1}^{m} S_{j, h} a_{m, j}^{-}=\delta_{h, m-1}(-1)^{m-1} \quad(0 \leq h \leq m-1),
\end{array}\right.
$$

we obtain the following condition:

$$
\left\{\begin{array}{l}
\widetilde{T}_{j, 0} a_{m, j, \nu}^{-, \mu}=r_{m, j, \nu}^{\mu} \\
\sum_{j=1}^{m} S_{j, h, 0} a_{m, j, 0}^{-\mu}=q_{h, m}^{\mu}
\end{array}\right.
$$

where

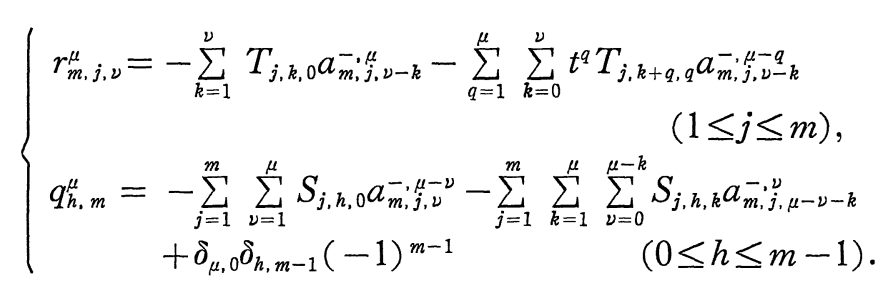

Here, we used a convention that we omit the term which has a factor with a negative index.

Next, we will give an analytic meaning to the previous formal argument. Before we start, it is convenient to introduce the following definition.

Definition. Let $f(t, x, \xi)$ be a function $C^{\infty}$ in $(-\infty, 0) \times \boldsymbol{R}_{x}^{n} \times$ $\left(\boldsymbol{R}_{\xi}^{n}-\{0\}\right)$ and $f^{*}(t, x, \xi)=\sum_{i=1}^{I} \sum_{j=1}^{J}(\log t)^{j} t^{\nu, j} j^{(x, \xi)} \sum_{r=0}^{\infty} f_{i, j, r}(x, \xi) t^{-r}$ be a formal series such that each $\nu_{i, j}(x, \xi)$ and $f_{i, j, r}(x, \xi)$ is a $\boldsymbol{C}$ valued function defined on $\boldsymbol{R}_{x}^{n} \times\left(\boldsymbol{R}_{\xi}^{n}-\{0\}\right)$. We denote by

$$
f(t, x, \xi) \sim f^{*}(t, x, \xi) \quad(t \rightarrow-\infty)
$$

if the following condition (i) holds.

(i) For each $p \in \boldsymbol{Z}_{+}$and a compact set $K \subset \boldsymbol{R}^{n}-\{0\}$, there exists a series $\left(\varepsilon_{N}\right)$ such that $\lim _{N \rightarrow \infty} \varepsilon_{N}=-\infty$ and

$$
\begin{aligned}
\mid D_{t}^{k} f(t, x, \xi) & -D_{t}^{k}\left(\sum_{i=1}^{I} \sum_{j=1}^{J}(\log t)^{j} t^{\nu_{i, j}(x, \xi)}\right. \\
& \left.\times \sum_{r=0}^{N} f_{i, j, r}(x, \xi) t^{-r}\right)\left.|\leq C| t\right|^{\varepsilon_{N}}
\end{aligned}
$$




$$
\left(t \leq-1, x \in \boldsymbol{R}^{n}, \xi \in K, N \in \mathbb{Z}_{+}\right)
$$

for $0 \leq k \leq p$, where the constant $C$ only depends on $p, K$ and $N$. If there exist $i_{0}, j_{0}\left(1 \leq i_{0} \leq I, 1 \leq j_{0} \leq J\right)$ such that

(3. 22) $\nu_{i_{0}, j_{0}}(x, \xi)-\nu_{i, j}(x, \xi) \geq 0\left(1 \leq i \leq I, 1 \leq j \leq J, x \in \boldsymbol{R}^{n}, \xi \in \boldsymbol{R}^{n}-\{0\}\right)$, we also denote $(3.21)$ by $f(t, x, \xi)=O\left(t^{\nu_{0}, j_{0}}{ }^{(x, \xi)}(\log t)^{J}\right)$.

Furthermore, we denote by

$$
f(t, x, \xi) \simeq f^{*}(t, x, \xi) \quad(t \rightarrow-\infty)
$$

if the following two conditions (ii) and (iii) hold.

(ii) Each $\nu_{i, j}(x, \xi)$ and $f_{i, j, r}(x, \xi)$ is $C^{\infty}$ in $\boldsymbol{R}_{x}^{n} \times\left(\boldsymbol{R}_{\xi}^{n}-\{0\}\right)$.

(iii) For each $p \in \boldsymbol{Z}_{+}$and a compact set $K \subset \boldsymbol{R}^{n}-\{0\}$, there exists a series $\left(\varepsilon_{N}\right)$ such that $\lim _{N \rightarrow \infty} \varepsilon_{N}=-\infty$ and

$$
\begin{aligned}
& \mid D_{t}^{k} D_{x, \xi}^{\alpha} f(t, x, \xi)-D_{t}^{k} D_{x, \xi}^{\alpha}\left(\sum_{i=1}^{I} \sum_{j=1}^{J}(\log t)^{j} t^{\nu_{i, j}(x, \xi)}\right. \\
& \left.\times \sum_{r=0}^{N} f_{i, j, r}(x, \xi) t^{-r}\right)\left.|\leq C| t\right|^{\varepsilon_{N}} \\
& \quad\left(t \leq-1, x \in \boldsymbol{R}^{n}, \xi \in K, N \in \mathbb{Z}_{+}\right),
\end{aligned}
$$

for $k+|\alpha| \leq p$, where the constant $C$ only depends on $p, K$ and $N$. In the case (3.22) holds, we also denote (3.23) by $f(t, x, \xi)$ $=\widehat{O}\left(t^{\nu_{i}, j_{0}{ }^{(x, \xi)}}(\log t)^{J}\right)$. In addition, we write $h(t, s, x, \xi)=O\left(t^{\nu(x, \xi)}(\log t)^{J}\right)$ - $O\left(s^{\mu(x, \xi)}(\log s)^{K}\right) \quad$ (respectively, $h(t, s, x, \xi)=\widehat{O}\left(t^{\nu(x, \xi)}(\log t)^{J}\right) \cdot \widehat{O}\left(s^{\mu(x, \xi)}\right.$ $\left.\left.(\log s)^{K}\right)\right)$ provided $h(t, s, x, \xi)$ is given by $h(t, s, x, \xi)=f(t, x, \xi) g(s$, $x, \xi)$ with $f(t, x, \xi)=O\left(t^{\nu(x, \xi)}(\log t)^{J}\right), \quad g(s, x, \xi)=O\left(s^{\mu(x, \xi)}(\log s)^{K}\right)$ (respectively, $f(t, x, \xi)=\hat{O}\left(t^{\nu(x, \xi)}(\log t)^{J}\right), g(s, x, \xi)=\hat{O}\left(s^{\mu(x, \xi)}(\log \mathrm{s})^{K}\right)$.)

We can prove the following lemma by a proof similar to that of Lemma 3.4 and 3.9 of Nakamura-Uryu [13].

Lemma 3. 4. For $s<0$,

$$
\widetilde{T}_{j, 0}=s^{(m-1) l-1} \sum_{k=0}^{m} s^{m-k} q_{k}(s, x, \xi) \partial_{s}^{m-k},
$$

where $q_{k}(s, x, \xi)(0 \leq k \leq m)$ are polynomials of $s^{-1}$ whose coefficients are $C^{\infty}$ in $\boldsymbol{R}_{x}^{n} \times\left(\boldsymbol{R}_{\xi}^{n}-\{0\}\right)$ such that

$$
q_{k}(-\infty, x, \xi) *=0 \quad(k \leq m-2),
$$

* $q_{k}(-\infty, x, \xi)=\lim _{s \rightarrow-\infty} q_{k}(s, x, \xi)$. 


$$
\begin{aligned}
& q_{m-1}(-\infty, x, \xi)=\sqrt{-1} G_{j}(x, \xi), \\
& q_{m}(-\infty, x, \xi)=-\sqrt{-1} H_{j}(x, \xi)+\sqrt{-1} l(m-1) G_{j}(x, \xi) .
\end{aligned}
$$

Moreover, there exist $Z_{j}^{-}(s, x, \xi)(1 \leq j \leq m)$ such that $\exp \left[-\sqrt{-1} s^{l+1}\right.$. $\left.(l+1)^{-1} \lambda_{j}(0, x, \xi)\right] Z_{j}^{-}(s, x, \xi)(1 \leq i \leq m)$ is a fundamental system of solutions for $M_{0}$ in $s<0$ and satisfy the asymptotic property:

$$
Z_{j}^{-}(s, x, \xi) \simeq f_{j}^{*}(s, x, \xi)=s^{\sigma_{j}(x, \xi)} \sum_{r=0}^{\infty} f_{j, r}(x, \xi) s^{-r}
$$

as $s \rightarrow-\infty$, where $f_{j, 0}(x, \xi) \equiv 1, \sigma_{j}(x, \xi)=-\mu_{j}(x, \xi)-l(m-1)$. In addition the equation $\widetilde{T}_{j, 0} v=f(s, x, \xi)$ with $f(s, x, \xi)=O\left(s^{\nu_{i_{0}}, j_{0}}{ }^{\left(x_{,} \xi\right)}(\log \right.$ $s)^{J}$ ) possesses a particular solution $v(s, x, \xi)$ such that $v(s, x, \xi)$ $=O\left(s^{\nu_{i_{0}}, j_{0}(x, \xi)-l(m-1)+1}(\log s)^{K}\right)$ for some $K \in \boldsymbol{Z}_{+}$.

Remark. (1) The leading term of the asymptotic expansion of $\widetilde{V}_{\bar{j}_{, m-1}}(s, x, \xi)$ as $s$ tends to $-\infty$ has the power $\sigma_{j}(x, \xi)$.

(2) In order to obtain a complete result for branching of singularities by discussing in the analytic category, we have to obtain similar estimates for $Z_{j}^{-}(s, x, \xi)$ and $v(s, x, \xi)$ in each sector which sits inside of $\boldsymbol{C}$ with angle less than $\pi(l+1)^{-1}$ and has a vertex at origin. This is possible if we replace the contour of (4.23) given in Coddington-Levinson [6] by that of the integral in p 316 of Nishimoto [15] and follow the argument given in [6].

Next lemma is a core of our construction.

Lemma 3.5. For each $\nu, \mu \in \boldsymbol{Z}_{+}, \alpha \in \boldsymbol{Z}_{+}^{2 n}$, the estimate

$$
\begin{aligned}
& \partial_{x, \xi}^{\alpha} a_{m, j, \nu}^{-, \mu}(t, s, x, \xi) \\
& \quad=O\left(t^{\mu_{j}(x, \xi)+\mu}(\log t)^{\Phi(\nu, \mu, \alpha)}\right) \cdot O\left(s^{-\mu_{j}(x, \xi)-l(m-1)+\nu}(\log s)^{\Psi(\nu, \mu, \alpha)}\right)
\end{aligned}
$$

for some $\Phi(\nu, \mu, \alpha), \Psi(\nu, \mu, \alpha) \in \boldsymbol{Z}_{+}$.

Proof. The proof heavily relies upon the fact $a_{m, j, \nu}^{-, \mu}(t, s, x, \xi)$ and $r_{m, j, \nu}^{u}(t, s, x, \xi)$ given in (3.20) can be obtained in the form:

$$
\begin{aligned}
& a_{m, j, \nu}^{-, \mu}(t, s, x, \xi)=\sum_{\gamma=1}^{\sigma(\mu, \nu)} g_{\gamma, m, j, \nu}^{\mu}(t, x, \xi) f_{\gamma, m, j, \nu}^{\mu}(s, x, \xi) \\
& r_{m, j, \nu}^{\mu}(t, s, x, \xi)=\sum_{\delta=1}^{\pi(\mu, \nu)} w_{\delta, m, j, \nu}^{\mu}(t, x, \xi) v_{\delta, m, j, \nu}^{\mu}(s, x, \xi) .
\end{aligned}
$$

Namely, how $f_{r, m, j, \nu}^{\mu}, g_{r, m, j, \nu}^{\mu}, v_{\delta, m, j, \nu}^{\mu}$ and $w_{\delta, m, j, \nu}^{\mu}$ are determined. We 
divide the proof into six steps. Steps 1 to 3 are devoted to the proof of the above fact and the rest to the proof of Lemma 3. 5 .

We prove the above fact by double induction on $\nu$ and $\mu$.

Step 1. The validity of the fact for $\mu=\nu=0$. In this case (3.19) becomes

$$
\left\{\begin{array}{l}
\widetilde{T}_{j, 0} a_{m, j, 0}^{-, 0}=0 \quad(1 \leq j \leq m), \\
\sum_{j=1}^{m} S_{j, h, 0} a_{m, j, 0}^{-, 0}=\delta_{h, m-1}(-1)^{m-1} \quad(0 \leq h \leq m-1) .
\end{array}\right.
$$

Hence (3.27) is obvious. Define $U_{m}^{-}$by (2.16). Then, it is easy to see from the proof of (3.6), $U_{m}^{-}$satisfies

$$
\left\{\begin{array}{l}
M_{0} U_{m}^{-}=0, \\
\left.D_{s}^{h} U_{m}^{-}\right|_{s=t}=\delta_{h, m-1}(-1)^{m-1} \quad(0 \leq h \leq m-1) .
\end{array}\right.
$$

The proof of Lemma 3. 1 reveals us that $U_{m}^{-}$satisfies (2.17). Hence, if we prove (2.18), we can define $a_{m, j, 0}^{-, 0}$ by $(2.15)$ and we will be done. Therefore, it is enough to prove (2.18).

We only prove (2.18) in the case $i=m$, because we can deal the other cases in the same way. Since $U_{i}^{-}(1 \leq i \leq m)$ and $U_{i}(1 \leq i \leq m)$ given by (2.9) are linearly independent, there is a relation

$$
\begin{aligned}
& { }^{t}\left[U_{1}^{-}(t, s, x, \xi), \cdots, U_{m}^{-}(t, s, x, \xi)\right] \\
& \quad={ }^{t} C(s, x, \xi)^{t}\left[U_{1}(t, x, \xi), \cdots, U_{m}(t, x, \xi)\right],
\end{aligned}
$$

where $C(s, x, \xi)$ is a matrix $C(s, x, \xi)=\left(C_{i, j}(s, x, \xi)\right)_{1 \leq i, j \leq m}$ Differentiating (3.30) up to $m-1$ times and set $t=s$, we have

$$
\begin{aligned}
& C(s, x, \xi)=\left(D_{s}^{h} U_{j}(s, x, \xi) ; \begin{array}{l}
h \downarrow 0, \cdots, m-1 \\
j \rightarrow 1, \cdots, m
\end{array}\right)^{-1} \\
& \quad=W\left(U_{1}, \cdots, U_{m} ; s, x, \xi\right)^{-1} \cdot\left(\tilde{U}_{j}^{h}(s, x, \xi) ; \begin{array}{l}
h \downarrow 0, \cdots, m-1 \\
j \rightarrow 1, \cdots, m
\end{array}\right),
\end{aligned}
$$

where $\tilde{U}_{j}^{h}(s, x, \xi)$ is the $(h+1, j)$-cofactor of the matrix $\left(D_{s}^{h} U_{j}(s\right.$, $\left.x, \xi): \begin{array}{l}h \downarrow 0, \cdots, m-1 \\ j \rightarrow 1, \cdots, m\end{array}\right)$ and $W\left(U_{1}, \cdots, U_{m} ; s, x, \xi\right)$ is the Wronskian of $U_{i}(s, x, \xi)(1 \leq i \leq m)$. From Liouville's formula

$$
W\left(U_{1}, \cdots, U_{m} ; s, x, \xi\right)=\exp \left[\sqrt{-1}(l+1)^{-1} \sum_{j=1}^{m} \lambda_{j}(0, x, \xi)\right] .
$$

Hence combining (3.30), (3.31) and (3.32), we obtain 


$$
\begin{aligned}
U_{m}^{-}(t, s, x, \xi)= & \sum_{j=1}^{m} \exp \left[-\sqrt{-1}(l+1)^{-1} \sum_{q=1}^{m} \lambda_{q}(0, x, \xi)\right] \\
& \times \tilde{U}_{j}^{m-1}(s, x, \xi) U_{j}(t, x, \xi) .
\end{aligned}
$$

Substitute

$$
\begin{aligned}
U_{k}^{h}(s, x, \xi) \equiv & D_{s}^{h} U_{k}(s, x, \xi) \\
= & \sum_{\mu=1}^{m} \exp \left[\sqrt{-1} s^{l+1}(l+1)^{-1} \lambda_{\mu}(0, x, \xi)\right] \\
& \cdot T_{-}^{(k, \mu)}(x, \xi) V_{\mu, h}^{-}(s, x, \xi)
\end{aligned}
$$

into

$$
\tilde{U}_{j}^{m-1}=\sum_{\sigma \in \mathscr{G}_{m}, \sigma(m)=j} \operatorname{sgn} \sigma U_{\sigma(1)}^{0} U_{\sigma(2)}^{1} \cdots U_{\sigma(m-1)}^{m-2},
$$

we have

$$
\begin{aligned}
& \tilde{U}_{j}^{m-1}(s, x, \xi)=\sum_{\sigma \in \mathscr{G}_{m},{ }^{\prime}(m)=j} \operatorname{sgn} \sigma \sum_{\mu(1)=1}^{m} \ldots \sum_{\mu(m-1)=1}^{m} \\
& T_{-}^{(o(1), \mu(1))}(x, \xi) \cdots T_{-}^{(\sigma(m-1), \mu(m-1))}(x, \xi) \\
& \times \exp \left[\sqrt{-1} s^{l+1}(l+1)^{-1} \sum_{q=1}^{m-1} \lambda_{\mu(q)}(0, x, \xi)\right] \\
& \times V_{\mu(1), 0}^{-}(s, x, \xi) \cdots V_{\mu(m-1), m-2}^{-}(s, x, \xi) \text {. }
\end{aligned}
$$

Here $\mathscr{G}_{m}$ is the permutation group of the ordered set $(1,2, \cdots, m)$. We can rewrite (3.36) in the form:

$$
\begin{aligned}
\tilde{U}_{j}^{m-1}(s, x, \xi)= & \sum_{\mu(m)=1}^{m}\left\{\sum_{\mu \in \mathscr{G}_{m}, \mu(m)=m-1} \operatorname{sgn} \mu \cdot V_{\mu(1), 0}^{-}(s, x, \xi) \cdots\right. \\
& \left.\cdots V_{\mu(m-1), m-2}^{-}(s, x, \xi)\right\}\left\{\sum_{\sigma \in \mathscr{G}_{m^{\prime}} \sigma(m)=j}\right. \\
& \operatorname{sgn}\left(\begin{array}{c}
\sigma(1), \cdots, \sigma(m) \\
\mu(1), \cdots, \mu(m)
\end{array}\right) \cdot \\
& \left.\cdot T_{-}^{(\sigma(1), \mu(1))}(x, \xi) \cdots T_{-}^{(\sigma(m-1), \mu(m-1))}(x, \xi)\right\} \\
& \times \exp \left[\sqrt{-1} s^{l+1}(l+1)^{-1} \cdot \sum_{q=1}^{m-1} \lambda_{\mu(q)}(0, x, \xi)\right] \\
= & \sum_{\mu(m)=1}^{m} \exp \left[\sqrt{-1} s^{l+1}(l+1)^{-1}\right. \\
& \left.\times \sum_{q=1}^{m-1} \lambda_{\mu(q)}(0, x, \xi)\right] \tilde{T}^{(j, \mu(m))}(x, \xi) \cdot \tilde{V}_{\mu(m), m-1}^{-}(s, x, \xi) .
\end{aligned}
$$

Then, substituting (3.37) into (3.33), we obtain the first equality of (2.18). The second equality of (2.18) can be easily proved by using (2. 10).

A similar argument entails the following fact which we use in Step 2: 
Let each $\tilde{U}_{i}(t, s, x, \xi)(1 \leq i \leq m)$ be the solution of the Cauchy problem:

$$
M_{0} \tilde{U}_{i}=0,\left.D_{s}^{h} \tilde{U}_{i}\right|_{s=t}=\delta_{h, i-1}(0 \leq h \leq m-1) .
$$

Also, let each $Y_{i}(s, x, \xi)(1 \leq i \leq m)$ be the solution of the Cauchy problem:

$$
M_{0} Y_{i}=0,\left.D_{s}^{h} Y_{i}\right|_{s=0}=\delta_{h, i-1}(0 \leq h \leq m-1) .
$$

Define $H_{-}^{(i, j)}(x, \xi)(1 \leq i, j \leq m)$ by

$$
\begin{aligned}
Y_{i}(s, x, \xi)= & \sum_{j=1}^{m} \exp \left[-\sqrt{-1} s^{l+1}(l+1)^{-1} \lambda_{j}(0, x, \xi)\right] \\
& \times H_{-}^{(i, j)}(x, \xi) Z_{j}^{-}(s, x, \xi) .
\end{aligned}
$$

Then, the previous argument which led to $(2.18)$ entails

$$
\begin{aligned}
\tilde{U}_{i}(t, s, x, \xi)= & \operatorname{det}\left(H_{-}^{(i, j)}(x, \xi)\right)_{1 \leq i, j \leq m} \\
& \times \sum_{j=1}^{m} \exp \left[\sqrt{-1}(l+1)^{-1}\left(t^{l+1}-s^{l+1}\right) \lambda_{j}(0, x, \xi)\right] \\
& \times Z_{j}^{-}(s, x, \xi) \tilde{Z}_{j, i-1}^{-}(t, x, \xi),
\end{aligned}
$$

where

$$
\begin{aligned}
& Z_{j, i-1}^{-}(t, x, \xi)=\exp \left[\sqrt{-1} t^{l+1}(l+1)^{-1} \lambda_{j}(0, x, \xi)\right] \\
& \quad \times D_{t}^{i-1}\left(\exp \left[\sqrt{-1} t^{l+1}(l+1)^{-1} \lambda_{j}(0, x, \xi)\right] \cdot Z_{j}^{-}(t, x, \xi)\right)
\end{aligned}
$$

and $\tilde{Z}_{\bar{j}, i-1}(t, x, \xi)$ is the $(i, j)$-cofactor of matrix

$$
\left(Z_{\overline{j, i}}(t, x, \xi) ; \begin{array}{l}
i \downarrow 0, \cdots, m-1 \\
j \rightarrow 1, \cdots, m
\end{array}\right) .
$$

Step 2. The validity of the fact for $\mu=\mu_{0}, \nu=0$ provided it is true for $\mu<\mu_{0}$. In this case (3.20) becomes

$$
\left\{\begin{aligned}
& r_{m, j, 0}^{\mu_{0}}=-\sum_{q=1}^{\mu_{0}} t^{q} T_{j, q, q} a_{m, j, 0}^{-, \mu_{j}-q} \quad(1 \leq j \leq m) \\
& q_{h, m}^{\mu_{0}}=-\sum_{j=1}^{m} S_{j, k, 0} \sum_{\nu=1}^{\mu_{0}} a_{m, j, \nu}^{-, \mu_{0}-\nu}-\sum_{j=1}^{m} \sum_{k=1}^{\mu_{0}} \sum_{\nu=0}^{\mu_{0}-k} S_{j, h, k} a_{m, j, \nu_{0}-\nu-k}^{-, \nu} \\
&(0 \leq h \leq m-1) .
\end{aligned}\right.
$$

Since each term of $T_{j, q, q}$ has the form $b(x, \xi) s^{\sigma} D_{s}^{u}$, (3.27) is obvious. So let's write $r_{m, j, 0}^{\mu_{0}}$ in the form (3.27) and let $\tilde{f}_{\delta, m, j, 0}^{\mu_{0}}(s, x, \xi)$ be a particular solution of

$$
\widetilde{T}_{j, 0} \tilde{f}_{\delta, m, j, 0}^{\mu_{0}}=v_{\delta, m, j, 0}^{\mu_{0}}(s, x, \xi) .
$$

Since $\widetilde{T}_{j, 0}$ is independent of $D_{x}$ and $D_{l}$, it is clear 


$$
\tilde{a}_{m, j, 0}^{\mu_{0}}(t, s, x, \xi)=\sum_{\delta=1}^{\pi\left(\mu_{0}, 0\right)} w_{\delta, m, j, 0}^{\mu_{0}}(t, x, \xi) \tilde{f}_{\delta, m, j, 0}^{\mu_{0}}(s, x, \xi)
$$

satisfies

$$
\widetilde{T}_{j, 0} \tilde{a}_{m, j, 0}^{\mu_{0}}=r_{m, j, 0}^{\mu_{0}}
$$

Hence, by setting

$$
\begin{aligned}
\tilde{a}_{m, 0}^{\mu_{0}}(t, s, x, \xi)= & \sum_{j=1}^{m} \exp \left[\sqrt{-1}(l+1)^{-1}\left(t^{l+1}-s^{l+1}\right) \lambda_{j}(0, x, \xi)\right] \\
& \times \tilde{a}_{m, j, 0}^{\mu_{0}}(t, s, x, \xi)
\end{aligned}
$$

$$
\begin{aligned}
\tilde{a}_{m, 0}^{-, \mu_{0}}(t, s, x, \xi)= & \sum_{j=1}^{m} \exp \left[\sqrt{-1}(l+1)^{-1}\left(t^{l+1}-s^{l+1}\right) \lambda_{j}(0, x, \xi)\right] \\
& \times a_{m, j, 0}^{-, \mu_{0}}(t, s, x, \xi)
\end{aligned}
$$

we have

$$
\left\{\begin{array}{l}
M_{0}\left(\tilde{a}_{m, 0}^{-, \mu_{0}}-\tilde{a}_{m, 0}^{\mu_{0}}\right)=0, \\
\left.D_{s}^{h}\left(\tilde{a}_{m, 0}^{-, \mu_{0}}-\tilde{a}_{m, 0}^{\mu_{0}}\right)\right|_{s=t}=q_{h, m}^{\mu_{0}}(t, x, \xi)-\left.D_{s}^{h} \tilde{a}_{m, 0}^{\mu_{0}}\right|_{s=t} \quad(0 \leq h \leq m-1) .
\end{array}\right.
$$

Hence, if we put

$$
\tilde{q}_{h, m}^{\mu_{0}}(t, x, \xi)=q_{h, m}^{\mu_{0}}(t, x, \xi)-\left.D_{s}^{h} \tilde{a}_{m, 0}^{\mu_{0}}\right|_{s=t},
$$

we obtain

$$
\text { (3.48) } \begin{aligned}
\tilde{a}_{m, 0}^{-, \mu_{0}}-\tilde{a}_{m, 0}^{\mu_{0}}= & \sum_{h=0}^{m-1} \tilde{q}_{h, m}^{\mu_{0}}(t, x, \xi) \tilde{U}_{h+1}(t, s, x, \xi) \\
= & \operatorname{det}\left(H_{-}^{(i, j)}(x, \xi)\right)_{1 \leq i, j \leq m} \\
& \sum_{j=1}^{m} \sum_{h=0}^{m-1} \exp \left[\sqrt{-1}(l+1)^{-1}\left(t^{l+1}-s^{l+1}\right) \lambda_{j}(0, x, \xi)\right] \\
& \times \tilde{q}_{h, m}^{\mu_{0}}(t, x, \xi) \tilde{Z}_{j, h}(t, x, \xi) Z_{j}^{-}(s, x, \xi)
\end{aligned}
$$

by using the fact we mentioned at the end of Step 1 .

Putting (3.44), (3.45) and (3.48) together, we obtain

$$
\begin{aligned}
\tilde{a}_{m, 0}^{-, \mu_{0}}(t, s, x, \xi)= & \sum_{j=1}^{m} \exp \left[\sqrt{-1}(l+1)^{-1}\left(t^{l+1}-s^{l+1}\right) \lambda_{j}(0, x, \xi)\right] \\
& \times\left\{\sum_{\delta=1}^{\pi\left(\mu_{0}, 0\right)} w_{\delta, m, j, 0}^{\mu_{0}}(t, x, \xi) \cdot \tilde{f}_{\delta, m, j, 0}^{\mu_{0}}(s, x, \xi)\right. \\
& +\sum_{h=0}^{m-1} \operatorname{det}\left(H_{-}^{(i, j)}(x, \xi)\right)_{1 \leq i, j \leq m} \tilde{q}_{h, m}^{\mu_{0}}(t, x, \xi) \\
& \left.\times \widetilde{Z}_{j, h}^{-}(t, x, \xi) Z_{j}^{-}(s, x, \xi)\right\} .
\end{aligned}
$$

Then, reminding $\widetilde{T}_{j, 0} V_{j}^{-}=0$ and $(3.43),(3.49)$ enables us to determine $a_{m, j, 0}^{-, \mu_{0}}$ by 


$$
\begin{aligned}
a_{m, j, 0}^{-, \mu_{0}}(t, s, x, \xi)= & \sum_{\delta=1}^{\pi\left(\mu_{0}, 0\right)} w_{\delta, m, j, 0}^{\mu_{0}}(t, x, \xi) \tilde{f}_{\delta, m, j, 0}^{\mu_{0}}(s, x, \xi) \\
& +\sum_{h=0}^{m-1} \operatorname{det}\left(H_{-}^{(i, j)}(x, \xi)\right)_{1 \leq i, j \leq m} \\
& \times \tilde{q}_{h, m}^{\mu_{0}}(t, x, \xi) \tilde{Z}_{\overline{j, h}}^{-}(t, x, \xi) Z_{j}^{-}(s, x, \xi) .
\end{aligned}
$$

Step 3. The validity of the fact for $\mu=\mu_{0}, \nu=\nu_{0}$ provided it is true for either $\mu<\mu_{0}$ or $\mu=\mu_{0}, \nu<\nu_{0}$. We can easily see this is true for (3.27). So write $r_{m, j, \nu_{0}}^{\mu_{0}}$ in the form (3.27) and let $f_{\delta, m, j, \nu_{0}}^{\mu_{0}}$ be a particular solution of

$$
\widetilde{T}_{j, 0} f_{\delta, m, j, \nu_{0}}^{\mu_{0}}=v_{\delta, m, j, \nu_{0}}^{\mu_{0}},
$$

then we define $a_{m, j, \nu_{0}}^{-, \mu_{0}}$ by

$$
a_{m, j, \nu_{0}}^{-, \mu_{0}}(t, s, x, \xi)=\sum_{\delta=1}^{\pi\left(\mu_{0}, \nu_{0}\right)} w_{\delta, m, j, \nu_{0}}^{\mu_{0}}(t, x, \xi) f_{\delta, m, j, \nu_{0}}^{\mu_{0}}(s, x, \xi) .
$$

This completes the proof of the fact.

Next we proceed to prove (3.25). This is also proved by double induction on $\mu$ and $\nu$. As before, the proof consists of three steps.

Step 4. The validity of (3.25) for $\mu=\nu=0$. Consider (2.15). We have from the proof of Lemma 3.9 of Nakamura-Uryu [13]

$$
V_{j}^{-}(t, x, \xi)=\hat{O}\left(t^{\mu_{j}(x, \xi)}\right) \quad(1 \leq j \leq m) .
$$

Since $\widetilde{V}_{j_{j}, m-1}(s, x, \xi)=\sum_{\sigma \in \mathscr{G}_{m^{*} \sigma(m)=j}} \operatorname{sgn} \sigma V_{\sigma(1), 0}^{-}(s, x, \xi) \cdots V_{\sigma(m-1), m-2}^{-}(s, x, \xi)$, (2.7) and (3.53) imply the estimate:

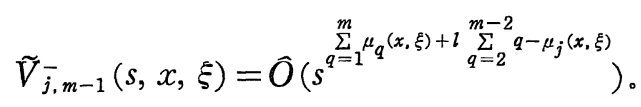

From the well-known relation $\sum_{q=1}^{m} \mu_{q}=-l\left(\begin{array}{c}m \\ 2\end{array}\right)=-l \sum_{q=1}^{m-1} q$, this becomes

$$
\widetilde{V}_{\bar{j}, m-1}(s, x, \xi)=\widehat{O}\left(s^{-\mu_{j}(x, \xi)-l(m-1)}\right) .
$$

Therefore, combining (2.15), (3.53) and (3.54), the estimate (3.25) holds for $\mu=\nu=0$ and any $\alpha \in \mathbb{Z}_{+}^{2 n}$.

A similar argument entails the following estimate which we use in Step 5:

$$
\left\{\begin{array}{l}
Z_{j}^{-}(s, x, \xi)=\hat{O}\left(s^{-\mu_{j}(x, \xi)-l(m-1)}\right) \\
\tilde{Z}_{\bar{j}^{-},-1}^{-}(t, x, \xi)=\hat{O}\left(t^{\mu_{j}(x, \xi)+l(m-1)-l(i-1)}\right)
\end{array} \quad(1 \leq i, j \leq m)\right.
$$

Step 5. The validity of (3.25) for $\mu=\mu_{0}, \nu=0$ provided it is true 
for $\mu<\mu_{0}$. From Lemma 3.2, we have

$$
r_{m, j, 0}^{\mu_{0}}=O\left(t^{\mu_{j}(x, \xi)+\mu_{0}}(\log t)^{\Phi^{\prime}\left(0, \mu_{0}, 0\right)}\right) O\left(s^{-\mu_{j}(x, \xi)-l(m-1)}(\log s)^{\Psi^{\prime}\left(0, \mu_{0}, 0\right)}\right)
$$

for some $\Phi^{\prime}\left(0, \mu_{0}, 0\right), \Psi^{\prime}\left(0, \mu_{0}, 0\right) \in \boldsymbol{Z}_{+}$. Thus, by Lemma 3. 4, $\tilde{a}_{m, j, 0}^{\mu_{0}}$ given by $(3.44)$ satisfies the estimate:

$$
\begin{aligned}
\tilde{a}_{m, j, 0}^{\mu_{0}}(t, s, x, \xi)= & O\left(t^{\mu_{j}(x, \xi)+\mu_{0}}(\log t)^{\Phi^{\prime}\left(0, \mu_{0}, 0\right)}\right) \\
& \times O\left(s^{-\mu_{j}(x, \xi)-l(m-1)}(\log s)^{\Psi^{\prime \prime}\left(0, \mu_{0}, 0\right)}\right),
\end{aligned}
$$

for some $\Psi^{\prime \prime}\left(0, \mu_{0}, 0\right) \in \boldsymbol{Z}_{+}$. From (3.56), we easily obtain the estimate:

$$
\begin{gathered}
\left.D_{s}^{h}\left(\exp \left[\sqrt{-1}(l+1)^{-1}\left(l^{l+1}-s^{l+1}\right) \lambda_{j}(0, x, \xi)\right] \tilde{a}_{m . j, 0}^{\mu_{0}}\right)\right|_{s=t} \\
=O\left(t^{\mu_{0}+h l-l(m-1)}(\log t)^{K\left(\mu_{0}, h, 0\right)}\right)
\end{gathered}
$$

for some $K\left(\mu_{0}, h_{0}, 0\right) \in \boldsymbol{Z}_{+}$.

Using Lemma 3. 3, $q_{h, m}^{\mu_{0}}$ given by (3.42) has the estimate:

$$
q_{h, m}^{\mu_{0}}(t, x, \xi)=O\left(t^{\mu_{0}+h l-l(m-1)}(\log t)^{\Theta\left(\mu_{0}, h, 0\right)}\right)
$$

for some $\Theta\left(\mu_{0}, h, 0\right) \in \boldsymbol{Z}_{+}$. Thus, combining (3.57) and (3.58), $\tilde{q}_{h, m}^{\mu_{0}}$ defined by (3.47) satisfies the estimate:

$$
\tilde{q}_{h, m}^{\mu_{0}}(t, x, \xi)=O\left(t^{\mu_{0}+h l-l(m-1)}(\log t)^{\Omega\left(\mu_{0}, h, 0\right)}\right)
$$

for some $\Omega\left(\mu_{0}, h, 0\right) \in \boldsymbol{Z}_{+}$. Therefore the validity of (3.25) for $|\alpha|=0$ follows from (3.44), (3.50), (3.55) and (3.59). The estimate (3.25) for general $\alpha \in \boldsymbol{Z}_{+}^{2 n}$ can be easily proved by induction on $|\alpha|$.

Step 6. The validity of (3.25) for $\mu=\mu_{0}, \nu=\nu_{0}$ provided it is true for either $\mu<\mu_{0}$ or $\mu=\mu_{0}, \nu<\nu_{0}$. The proof can be done much easier than Step 5 .

Q. E. D.

Now, reminding the semi-homogeneity of $a_{m, j, \nu}^{-, \mu}$, we can interpret (3.25) in terms of our symbol class.

\section{Lemma 3. 6.}

$$
\begin{gathered}
a_{m, j, \nu}^{-, \mu}(t, s, x, \xi) \in \tilde{S}^{-}\left[2 \varepsilon(l+1)^{-1}-(m-1), m_{j}^{+}+\mu+\varepsilon,\right. \\
\left.m_{j}^{-}-l(m-1)+\nu+\varepsilon\right] \quad\left(1 \leq j \leq m, \nu, \mu \in Z_{+}\right)
\end{gathered}
$$

for any $\varepsilon>0$.

Proof. Let $p, q \in \boldsymbol{Z}_{+}, \alpha, \beta \in \boldsymbol{Z}_{+}^{n}$. Since $D_{t}^{p} D_{s}^{q} D_{x}^{\alpha} D_{\xi}^{\beta} a_{m, j, \nu}^{-, \mu}$ is semihomogeneous of degree $-(l+1)^{-1}(\nu+\mu+m-p-q-1)-|\beta|$, we have 
(3.61)

$$
\begin{aligned}
& D_{t}^{p} D_{s}^{q} D_{x}^{\alpha} D_{\xi}^{\beta} a_{m, j, \nu}^{-, \mu}(t, s, x, \xi)=|\xi|^{(l+1)^{-1}(p+q-\nu-\mu-m+1)-|\beta|} \\
& \quad \times D_{t}^{p} D_{s}^{q} D_{x}^{\alpha} D_{\xi}^{\beta} a_{m, j, \nu}^{-, \mu}\left(|\xi|^{(l+1)^{-1}} t,|\xi|^{(l+1)^{-1}} \mathrm{~s}, x,|\xi|^{-1} \xi\right) .
\end{aligned}
$$

From (3.25) and (3.61), we have

$$
\begin{aligned}
&\left|D_{t}^{p} D_{s}^{q} D_{x}^{\alpha} D_{\xi}^{\beta} a_{m, \mu}^{-, \mu \nu}(t, s, x, \xi)\right| \\
&\left.\leq C^{\prime}|\xi|^{(l+1)^{-1}(p+q-\nu-\mu-m+1)-|\beta|}\left(1+|\xi|^{(l+1)}\right)^{-1}|t|\right)^{R e \mu_{j}(x, \xi)+\mu-p+\varepsilon} \\
& \\
& \times\left(1+|\xi|^{(l+1)^{-1}}|s|\right)^{-R e \mu_{j}(x, \xi)-l(m-1)+\nu-q+\varepsilon} \\
& \leq C(1+|\xi|)^{2 \varepsilon(l+1)^{-1}-(m-1)-|\beta|}\left(|\xi|^{-1}+|t|^{l+1}\right)^{(l+1)^{-1}\left(m_{j}^{+}+\mu-p+\varepsilon\right)} \\
& \quad \times\left(|\xi|^{-1}+|s|^{l+1}\right)^{(l+1)^{-1}\left(m_{j}^{-}-l(m-1)+\nu-q+\varepsilon\right)} \\
& \quad\left(-T_{0} \leq s, t \leq 0, x \in \boldsymbol{R}^{n},|\xi| \geq 1\right)
\end{aligned}
$$

for some constant $C, C^{\prime}$.

Q. E. D.

For further argument, we need some properties of our symbol classes.

Lemma 3. 7. The following properties hold for $\mu, \kappa, \lambda \in \boldsymbol{R}$.

(1) $\quad \tilde{S}^{ \pm}[\mu, \kappa] \subset \tilde{S}^{ \pm}\left[\mu^{\prime}, \kappa^{\prime}\right]$

if $\mu \leq \mu^{\prime}, \quad \mu-\kappa(l+1)^{-1} \leq \mu^{\prime}-\kappa^{\prime}(l+1)^{-1}$.

(2) $\quad \tilde{S}^{ \pm}[\mu, \kappa, \lambda] \subset \tilde{S}^{ \pm}\left[\mu^{\prime}, \kappa^{\prime}, \lambda^{\prime}\right]$

if either

$$
\begin{array}{r}
\mu \leq \mu^{\prime}, \mu-\kappa(l+1)^{-1} \leq \mu^{\prime}-\kappa^{\prime}(l+1)^{-1}, \lambda=\lambda^{\prime} \\
\text { or } \mu \leq \mu^{\prime}, \mu-\lambda(l+1)^{-1} \leq \mu^{\prime}-\lambda^{\prime}(l+1)^{-1}, \kappa=\kappa^{\prime} .
\end{array}
$$

(3) $\quad b(t, s, x, \xi) \in \tilde{S}^{ \pm}[\mu, \kappa, \lambda]$

implies

$$
b(t, t, x, \xi) \in \tilde{S}^{ \pm}[\mu, \kappa+\lambda]
$$

and

$$
b(0, s, x, \xi) \in \tilde{S}^{ \pm}\left[\mu-(l+1)^{-1} \kappa, \lambda\right] .
$$

(4) Let $b(t, x, \xi) \in \tilde{S}^{ \pm}[\mu, \kappa]$

and

$$
\chi^{ \pm} \in C^{\infty}\left(R^{1}\right) \text { satisfy } \chi^{ \pm}(\theta)=0\left(0 \leq \pm \theta \leq \frac{1}{2}\right), \quad=1( \pm \theta \geq 1) .
$$

Then, for each $p \in Z_{+}, \alpha \in \mathrm{Z}_{+}^{n}, \beta \in \mathrm{Z}_{+}^{n}$, we have

$$
\begin{gathered}
\left|D_{t}^{p} D_{x}^{\alpha} D_{\xi}^{\beta}\left\{\chi^{ \pm}\left(|\xi|^{(l+1)^{-1}} t\right) b(t, x, \xi)\right\}\right| \leq C(1+|\xi|)^{\mu-|\beta|}|t|^{\kappa-p} \\
\left(0 \leq \pm t \leq T_{0}, x \in \boldsymbol{R}^{n},|\xi| \geq 1\right)
\end{gathered}
$$

where $C$ depends only on $p, \alpha, \beta$. 
(5) Let $b(t, s, x, \xi) \in \tilde{S}^{ \pm}[\mu, \kappa, \lambda]$ and $\chi^{ \pm}$be as above. Then, for each $p, q \in Z_{+}, \alpha, \beta \in Z_{+}^{n}$, we have

$$
\left\{\begin{array}{c}
\left|D_{t}^{p} D_{s}^{q} D_{x}^{\alpha} D_{\xi}^{\beta}\left\{\chi^{ \pm}\left(|\xi|^{(l+1)^{-1}} t\right) b(t, s, x, \xi)\right\}\right| \\
\leq C(1+|\xi|)^{\mu-|\beta|}\left(|\xi|^{-1}+|s|\right)^{(l+1)^{-1}(\lambda-q)}|t|^{\kappa-p} \\
\left|D_{t}^{p} D_{s}^{q} D_{x}^{\alpha} D_{\xi}^{\beta}\left\{\chi^{ \pm}\left(|\xi|^{(l+1)^{-1}} s\right) \quad b(t, s, x, \xi)\right\}\right| \\
\leq C(1+|\xi|)^{\mu-|\beta|}\left(|\xi|^{-1}+|t|\right)^{(l+1)^{-1}(\kappa-p)}|s|^{\lambda-q} \\
\left(0 \leq \pm s, \pm t \leq T_{0}, x \in \boldsymbol{R}^{n}, \quad|\xi| \geq 1\right)
\end{array}\right.
$$

where $C$ depends only on $p, q, \alpha, \beta$.

(6) Let $b_{q}(t, x, \xi) \in \tilde{S}^{ \pm}[\mu, \kappa+q]\left(q \in Z_{+}\right)$, then there exists $b(t, x$, $\xi) \in \tilde{S}^{ \pm}[\mu, \kappa]$ such that

$$
b-\sum_{q<N} b_{q} \in \tilde{S}^{ \pm}[\mu, \kappa+N]
$$

for every $N \in \boldsymbol{N}$.

(7) Let $b_{q}(t, s, x, \xi) \in \tilde{S}^{ \pm}[\mu, \kappa+q, \lambda]$ (respectively $\in \tilde{S}^{ \pm}[\mu, \kappa, \lambda+q]$ ) $\left(q \in \mathbb{Z}_{+}\right)$, then there exists $b(t, s, x, \xi) \in \tilde{S}^{ \pm}[\mu, \kappa, \lambda]$ such that

$b-\sum_{q<N} b_{q} \in \tilde{S}^{ \pm}[\mu, \kappa+N, \lambda]$ (respectively, $\in \tilde{S}^{ \pm}[\mu, \kappa, \lambda+N]$ ) for every $N \in N$.

(8) Let $b_{q}(t, s, x, \xi) \in \tilde{S}^{ \pm}[\mu, \kappa+q, \infty]\left(q \in \boldsymbol{Z}_{+}\right)$, then there exists $b(t, s, x, \xi) \in \tilde{S}^{ \pm}[\mu, \kappa, \infty]$ such that

$$
b-\sum_{q<N} b_{q} \in \tilde{S}^{ \pm}[\mu, \kappa+N, \infty]
$$

for every $N \in N$.

(9) Let $b_{q}(t, s, x, \xi) \in \widetilde{S}^{-}[\mu-q, \infty]$ (respectively, $\tilde{S}^{ \pm}[\mu-q, \kappa, \infty]$ ) $\left(q \in Z_{+}\right)$, then there exists $b(t, s, x, \xi) \in \tilde{S}^{-}[\mu, \infty]$ (respectively, $\tilde{S}^{ \pm}[\mu$, $\kappa, \infty])$ such that

$$
b-\sum_{q<N} b_{q} \in \widetilde{S}^{-}[\mu-N, \infty] \text { (respectively, } \in \tilde{S}^{ \pm}[\mu-N, \kappa, \infty] \text { ) }
$$

for every $N \in \mathbb{N}$.

(10) Let $b(t, x, \xi) \in \tilde{S}^{ \pm}[\mu, \infty]$ (respectively, $b(t, s, x, \xi) \in \tilde{S}^{ \pm}[\mu, \infty$, 2], respectively $\left.b(t, s, x, \xi) \in \tilde{S}^{ \pm}[\mu, \kappa, \infty]\right)$, then there exists $c(t, x, \xi)$ $\in \tilde{S}^{ \pm}[\mu, \infty]$ (respectively, $c(t, s, x, \xi) \in \tilde{S}^{ \pm}[\mu, \infty, \lambda]$, respectively $c(t, s$, $x, \xi) \in \tilde{S}^{ \pm}[\mu, \kappa, \infty]$ ) such that it is flat at $t=0$ (respectively, $t=0$, respectively $s=0)$ and $b-c \in S^{ \pm}[-\infty]$.

(11) Let $b(t, s, x, \xi) \in \tilde{S}^{-}[\mu, \infty, \lambda]$, then there exists $c(t, s, x, \xi)$ $\in \widetilde{S}^{-}[\mu, \infty]$ such that $b-c \in S^{ \pm}[-\infty]$. 
Proof. (1), (2), (3), (4), (5) follow from the definitions of our symbol classes. Consult Prop. 3.13 in [25] or Prop. 1.8 in [26] for the proof of (6) and (7), and consult Prop. 1.18, Remark 1.19 in [26] for the proof of (9).

To prove (8), let $\chi^{ \pm} \in C^{\infty}\left(\mathbb{R}^{1}\right)$ be the one defined in (4) and define $\tilde{\chi}_{r}^{ \pm}(t, \xi)=\chi^{+}\left(\gamma^{-1}|\xi|\right)\left(1-\chi^{ \pm}\left(\gamma t^{l+1}\right)\right)(\gamma \geq 1)$. Now, for each $k \in \mathbb{Z}_{+}$, choose a series $\left(\gamma_{j, k}\right)$ such that $\gamma_{j, k} \rightarrow \infty(j \rightarrow \infty), \gamma_{j, k} \leq \gamma_{j, k+1}\left(j, k \in \mathbb{Z}_{+}\right)$ and $\left\{2 \tilde{\chi}_{r_{j, k}}^{j_{ \pm}} b_{j}\right\}_{j \geq N}$ is bounded in $\tilde{S}^{ \pm}[\mu, \kappa, \lambda+N-2]$ for each $N \in \mathbb{Z}_{+}$. Then, it is not difficult to see $b(t, s, x, \xi)$ can be given by $b=\sum_{j=0}^{\infty} \tilde{\chi}_{r_{j, j}}^{ \pm} b_{j}$.

Next we proceed to prove (10). Since the other cases can be proved in the same way, we only sketch the proof for (10) in the case $b(t, s, x, \xi) \in \tilde{S}^{ \pm}[\mu, \kappa, \infty]$. Let $\chi^{ \pm} \in C^{\infty}\left(\boldsymbol{R}^{1}\right)$ be as above. Set $\tilde{\chi}_{r}^{ \pm}(s)=1-\chi^{ \pm}(\gamma s)(\gamma \geq 1), b_{j}(t, s, x, \xi)=(j !)^{-1} s^{j} \partial_{s}^{j} b(t, 0, x, \xi) \in S^{ \pm}[-\infty]$ $\left(j \in N_{+}\right)$. Choose a series $\left(\gamma_{j}\right)$ so that $\left\{2^{j} \tilde{\chi}_{r_{j}}^{ \pm}(s) b_{j}(t, s, x, \xi)\right\}_{j>N}$ is bounded in $S^{ \pm}[-N]$ for each $N \in \mathbb{Z}_{+}$. Then, it is not difficult to see $c(t, s, x, \xi)$ can be given by $c=b-\sum_{j=0}^{\infty} \tilde{\chi}_{r_{j}}^{ \pm} b_{j}$.

(11) follows from (10) and the fact: If $c(t, s, x, \xi) \in \tilde{S^{-}}[\mu, \infty, \lambda]$ and is flat at $t=0$, then $c \in \widetilde{S}^{-}[\mu, \infty]$. This can be proved by expressing $c$ in terms of Taylor's remainder formula and estimating it.

Q. E. D.

From Lemma 3.7, for each $j(1 \leq j \leq m)$, there exist $a_{m, j}^{-, \mu}(t, s, x, \xi)$ $\in \tilde{S}^{-}\left[2 \varepsilon(l+1)^{-1}-(m-1), m_{j}^{+}+\mu+\varepsilon, m_{j}^{-}-l(m-1)+\varepsilon\right] \quad\left(\mu \in \mathbb{Z}_{+}, \varepsilon>0\right)$ and $a_{m, j}^{-}(t, s, x, \xi) \in \tilde{S}^{-}\left[2 \varepsilon(l+1)^{-1}-(m-1), m_{j}^{+}+\varepsilon, m_{j}^{-}-l(m-1)+\varepsilon\right]$ $(\varepsilon>0)$ such that

$$
\begin{gathered}
a_{m, j}^{-, \mu}-\sum_{\nu<N} a_{m, j, \nu}^{-, \mu} \in \tilde{S}^{-}\left[2 \varepsilon(l+1)^{-1}-(m-1), m_{j}^{+}+\mu+\varepsilon,\right. \\
\left.m_{j}^{-}-l(m-1)+N+\varepsilon\right], \\
a_{m, j}^{-}-\sum_{\mu<N} a_{m, j}^{-, \mu} \in \tilde{S}^{-}\left[2 \varepsilon(l+1)^{-1}-(m-1), m_{j}^{+}+N+\varepsilon,\right. \\
\left.m_{j}^{-}-l(m-1)+\varepsilon\right]
\end{gathered}
$$

for every $N \in \boldsymbol{N}$. Unfortunately, $a_{m, j}^{-}(t, s, x, \xi)(1 \leq j \leq m)$ defined above do not satisfy $T_{j} a_{m, j}^{-}=0(1 \leq j \leq m), \sum_{j=1}^{m} S_{j, h} a_{m, j}^{-}=\delta_{h, m-1}(-1)^{m-1} \quad(0 \leq h \leq$ $m-1)$. However, the following lemma reveals us that the errors are tame enough to compensate.

Lemma 3.8.

(1) $\quad r_{m, j}=T_{j} a_{m, j}^{-} \in \tilde{S}^{-}\left[2 \varepsilon(l+1)^{-1}, m_{j}^{+}+\varepsilon, m_{j}^{-}-l(m-1)+\varepsilon\right](1 \leq j \leq m)$ 
for any $\varepsilon>0$.

(2) $\sum_{j=1}^{m} S_{j, h} a_{m, j}^{-}-\delta_{h, m-1}(-1)^{m-1} \in \tilde{S}^{-}\left[2 \varepsilon(l+1)^{-1}-(m-1)+h, \infty\right]$ $(0 \leq h \leq m-1)$

for any $\varepsilon>0$.

(3) For each $N \in \boldsymbol{N}_{+}$, there exists $r_{m, j, N} \in \tilde{S}^{-}\left[2 \varepsilon(l+1)^{-1}, m_{j}^{+}+\varepsilon, \infty\right]$ $(\varepsilon>0)$ such that

$$
r_{m, j}-r_{m, j, N} \in \tilde{S}^{-}\left[2 \varepsilon(l+1)^{-1}, m_{j}^{+}+N+1+\varepsilon, m_{j}^{-}-l(m-1)-m+\varepsilon\right]
$$

Proof. The proof of (1) and (2) are essentially the same. The one for (1) is rather complicated. So we only illustrate the proofs of (1) and (3) simultaneously.

Let $J, N \in N_{+}$. Rewrite $T_{j} a_{\bar{m}, j}$ in the form:

$$
\begin{aligned}
T_{j} a_{m, j}^{-}= & T_{j}\left(a_{m, j}^{-}-\sum_{\mu=0}^{N} a_{m, j}^{-, j}\right) \\
& +\left\{T_{j} \sum_{\mu=0}^{N}\left(a_{m, j}^{-, \mu}-\sum_{\nu=0}^{J} a_{m, j, \nu}^{-, \mu}\right)+T_{j} \sum_{\nu=0}^{N} \sum_{\nu=0}^{J} a_{m, j, \nu}^{-, \mu}\right\} .
\end{aligned}
$$

Here, reminding (3.65) and each term of $T_{j}$ includes $D_{s}$ at most $m$ and is at most homogeneous of degree $m-1$, we see the first term belongs to $\tilde{S}^{-}\left[2 \varepsilon(l+1)^{-1}, m_{j}^{+}+N+1+\varepsilon, m_{j}^{-}-l(m-1)-m+\varepsilon\right](\varepsilon>0)$. To consider the second term, define $\check{a}_{m, j, \nu}^{-, \mu}$ and $\breve{T}_{j, k, q}$ as follows.

$$
\begin{aligned}
& \check{a}_{m, j, \nu}^{-, \mu}= \begin{cases}a_{m, j, \nu}^{-, \mu} & (0 \leq \nu \leq J, 0 \leq \mu \leq N), \\
a_{m, j}^{-, \mu}-\sum_{\nu=0}^{J} a_{m, j, \nu}^{-, \mu} & (\nu=J+1,0 \leq \mu \leq N), \\
0 & (\nu \geq J+2 \text { or } \mu \geq N+1)\end{cases} \\
& \check{T}_{j, k, q}= \begin{cases}T_{j, k, q} & (0 \leq k \leq J+N, 0 \leq q \leq k), \\
\check{T}_{j, J+N+1, q} & (k=J+N+1,0 \leq q \leq J+N+1), \\
0 & (k \geq J+N+2) .\end{cases}
\end{aligned}
$$

Then, it is clear $T_{j}$ is written in the form:

$$
T_{j}=\sum_{k=0}^{\infty} \sum_{q=0}^{k} t^{q} \breve{T}_{j, k, q}
$$

Now, if we remind the argument which led to (3.19), (3.20), we have

$$
\begin{gathered}
T_{j} \sum_{\mu=0}^{N}\left(a_{m, j}^{-, \mu}-\sum_{\nu=0}^{J} a_{m, j, \nu}^{-, \mu}\right)+T_{j} \sum_{\mu=0}^{N} \sum_{\nu=0}^{J} a_{m, j, \nu}^{-, \mu} \\
=\sum_{\mu=0}^{N} \sum_{\nu=0}^{\infty}\left(\sum_{k=0}^{\nu} \sum_{q=0}^{\mu} t^{q} \check{T}_{j, k+q, q} \check{a}_{m, j, \nu-k}^{-, \mu-q}\right)
\end{gathered}
$$




$$
+\sum_{\mu=N+1}^{\infty} \sum_{\nu=0}^{\infty}\left(\sum_{k=0}^{\nu} \sum_{q=0}^{\mu} t^{q} \check{T}_{j, k+q, q} \check{a}_{m, j, \nu-k}^{-, \mu-q}\right) \text {. }
$$

Here we note that Lemma 3.2 and its proof tell us $\breve{T}_{j, k+q, q}$ sends $\tilde{S}^{-}[\gamma, \kappa, \lambda]$ into $\tilde{S}^{-}[\gamma+(m-1), \kappa, \lambda+l(m-1)+k-1]$. Since we have

$$
\begin{gathered}
\check{a}_{m, j, \nu-k}^{-, \mu-q} \in \tilde{S}^{-}\left[2 \varepsilon(l+1)^{-1}-(m-1), m_{j}^{+}+\mu-q+\varepsilon,\right. \\
\left.m_{j}^{-}-l(m-1)+\nu-k+\varepsilon\right] \quad(\varepsilon>0)
\end{gathered}
$$

from (3.60), (3.64) and (3.68), the second term on the right hand side of (3.71) belongs to $\tilde{S}^{-}\left[2 \varepsilon(l+1)^{-1}, m_{j}^{+}+N+1+\varepsilon, m_{j}^{-}-1+\varepsilon\right] \subset$ $\tilde{S}^{-}\left[2 \varepsilon(l+1)^{-1}, m_{j}^{+}+N+1+\varepsilon, m_{j}^{-}-l(m-1)-m+\varepsilon\right](\varepsilon>0)$. So put

$$
r_{m, j, N}=\sum_{\mu=0}^{N} \sum_{\nu=0}^{\infty} \sum_{k=0}^{\nu} \sum_{q=0}^{\mu} t^{q} \breve{T}_{j, k+q, q} \check{a}_{m, j, \nu-k}^{-, \mu-q}
$$

and prove that this is the desired one. Rewrite (3.73) in the form:

$$
\begin{aligned}
r_{m, j, N} & =\sum_{\mu=0}^{N} \sum_{\nu=0}^{J} \sum_{k=0}^{\nu} \sum_{q=0}^{\mu} t^{q} \check{T}_{j, k+q, q} \check{a}_{m, j, \nu-k}^{-, \mu-q} \\
& +\sum_{\nu=0}^{N} \sum_{\nu=J+1}^{\infty} \sum_{k=0}^{\nu} \sum_{q=0}^{\mu} t^{q} \breve{T}_{j, k+q, q} \check{a}_{m, j, \nu-k}^{-, \mu-q} .
\end{aligned}
$$

The first term of (3.74) is zero because of (3.19), (3.20), (3.68) and (3.69). The second term belong to $\tilde{S}^{-}\left[2 \varepsilon(l+1)^{-1}, m_{j}^{+}+\varepsilon, m_{j}^{-}\right.$ $+\mathrm{J}+\varepsilon]$ which can be seen by the argument applied to the second term of (3. 71). Since $J \in \boldsymbol{Z}_{+}$is arbitrary, $r_{m, j, N} \in \tilde{S}^{-}\left[2 \varepsilon(l+1)^{-1}\right.$, $\left.m_{j}^{+}+\varepsilon, \infty\right](\varepsilon>0)$. Hence the assertions (1) and (3) are valid.

Q. E. D.

Furthermore, $r_{m, j}$ admits the following decomposition.

Lemma 3. 9. For each $j(1 \leq j \leq m)$, there exist $r_{m, j}^{0}(t, s, x, \xi)$ $\in \tilde{S}^{-}\left[2 \varepsilon(l+1)^{-1}, m_{j}^{+}+\varepsilon, \infty\right], r_{m, j}^{1}(t, s, x, \xi) \in \widetilde{S}^{-}\left[2 \varepsilon(l+1)^{-1}, \infty\right], r_{m, j}^{2}(t, s$, $x, \xi) \in S^{-}[-\infty]$ such that

$$
r_{m, j}=r_{m, j}^{0}+r_{m, j}^{1}+r_{m, j}^{2} \text { and } r_{m, j}^{0} \text { is flat at } s=0 \text {. }
$$

Proof. According to Lemma 3.8, for each $N \in \mathbb{Z}_{+}$, there exists $r_{m, j, N} \in \tilde{S}^{-}\left[2 \varepsilon(l+1)^{-1}, m_{j}^{+}+\varepsilon, \infty\right]$ with the property (3.66). Hence

$$
\begin{aligned}
r_{m, j, k+1}-r_{m, j, k} & =\left(r_{m, j, k+1}-r_{m, j}\right)+\left(r_{m, j}-r_{m, j, k}\right) \\
& \in \tilde{S}^{-}\left[2 \varepsilon(l+1)^{-1}, m_{j}^{+}+k+1+\varepsilon, \infty\right]\left(k \in \mathbb{Z}_{+}\right) .
\end{aligned}
$$

Then, from Lemma 3. 7, there exists $\tilde{r}_{m, j}^{1}(t, s, x, \xi) \in \tilde{S}^{-}\left[2 \varepsilon(l+1)^{-1}\right.$, $\left.m_{j}^{+}+1+\varepsilon, \infty\right]$ such that

$$
\tilde{r}_{m, j}^{1}-\sum_{k=0}^{N}\left(r_{m, j, k+1}-r_{m, j, k}\right) \in \tilde{S}^{-}\left[2 \varepsilon(l+1)^{-1}, m_{j}^{+}+N+2+\varepsilon, \infty\right] .
$$


Clearly, $r_{m, j, 0}+\tilde{r}_{m, j}^{1} \in \tilde{S}^{-}\left[2 \varepsilon(l+1)^{-1}, m_{j}^{+}+\varepsilon, \infty\right]$. From this and Lemma 3. 7, there exists $r_{m, j}^{0}(t, s, x, \xi) \in \tilde{S}^{-}\left[2 \varepsilon(l+1)^{-1}, m_{j}^{+}+\varepsilon, \infty\right]$ such that it is flat at $s=0$ and

$$
r_{m, j}^{0}-\left(r_{m, j, 0}+\tilde{r}_{m, j}^{1}\right) \in S^{-}[-\infty] .
$$

Then the assertion follows from (3.77), (3.78), Lemma 3.7 and

$$
\begin{aligned}
r_{m, j}-r_{m, j}^{0} & =\left(r_{m, j}-r_{m, j, 0}\right)+\sum_{k=0}^{N}\left(r_{m, j, k}-r_{m, j, k+1}\right) \\
& +\left\{\sum_{k=0}^{N}\left(r_{m, j, k+1}-r_{m, j, k}\right)-\tilde{r}_{m, j}^{1}\right\}+\left\{\left(r_{m, j, 0}+\tilde{r}_{m, j}^{1}\right)-r_{m, j}^{0}\right\} \\
& =\left(r_{m, j}-r_{m, j, N+1}\right)+\left\{\sum_{k=0}^{N}\left(r_{m, j, k+1}-r_{m, j, k}\right)-\tilde{r}_{m, j}^{1}\right\} \\
& +\left\{\left(r_{m, j, 0}+\tilde{r}_{m, j}^{1}\right)-r_{m, j}^{0}\right\} \\
& \in \tilde{S}^{-}\left[2 \varepsilon(l+1)^{-1}, \infty, m_{j}^{-}-l(m-1)-m+\varepsilon\right] . \quad \text { Q. E. D. }
\end{aligned}
$$

Next, by making use of Lemma 3.8 and 3.9, we show the usual integration procedure for the transport operator enables us to construct the compensating factors $\tilde{a}_{m, j}^{-}, \tilde{a}_{m, j}^{-}$which compensate the errors $r_{m, j}^{0}, \quad r_{m, j}^{1}, \sum_{j=1}^{m} S_{j, h} a_{m, j}^{-}-\delta_{h, m-1}(-1)^{m-1}$.

First we decompose the transport operator $T_{j}$ and $S_{j, h}$ into homogeneous parts. Taking account of the assumptions (A. 1)-(A. 3) and $P_{m}\left(s, \nabla_{\xi} \phi_{j},-\partial_{s} \phi_{j}, \xi\right)=0$, a proof analogous to that of Lemma 3.2 and 3.3 entails the following two lemmas.

\section{Lemma 3. 10.}

$$
T_{j} \approx \sum_{k=0}^{\infty} H_{j, k}
$$

where $H_{j, k}$ is homogeneous of degree $m-k-1$. Especially, $H_{j, 0}$ has the form:

$$
\begin{aligned}
H_{j, 0}= & s^{(m-1) l-1} A_{j, 0}(t, s, x, \xi)\left\{s \left(D_{s}+\sum_{k=1}^{m} A_{j, 0}(t, s, x, \xi)^{-1}\right.\right. \\
& \left.\left.\times A_{j, k}(t, s, x, \xi) D_{\xi_{k}}\right)+A_{j, 0}(t, s, x, \xi)^{-1} B_{j}(t, s, x, \xi)\right\},
\end{aligned}
$$

where $A_{j, k} \in S^{-}[m](1 \leq k \leq n)$ and $A_{j, 0}, B_{j} \in S^{-}[m-1]$ are semihomogeneous of degree $m$ and $m-1$. Also, $A_{j, k}(0 \leq k \leq n)$ are real valued and $A_{j, 0}$ satisfies

$$
\left|A_{j, 0}(t, s, x, \xi)\right| \geq C|\xi|^{m-1} \quad\left(-T_{0} \leq s, t \leq 0, \quad x, \xi \in \boldsymbol{R}^{n}\right),
$$

for some $C>0$. Here the meaning of the symbol " $\approx$ " is as follows. For each $N \in Z_{+}$, define $\hat{H}_{j, N+1}$ by 


$$
T_{j}=\sum_{k=0}^{N} H_{j, k}+\hat{H}_{j, N+1}
$$

then the effect of applying $\hat{H}_{j, N+1}$ to an element of one of our symbol classes is at most multiplying an element of $S^{-}[m-N-2]$.

\section{Lemma 3. 11}

$$
S_{j, h}=\sum_{k=0}^{h} M_{j, h, k}
$$

where $M_{j, h, k}$ is homogeneous of degree $h-k$ and $M_{j, h, 0}$ is given by

$$
M_{j, h, 0}=\left(-t^{l} \lambda_{j}(t, x, \xi)\right)^{h} .
$$

Now, let $\Xi(t, s, x, \xi)=\left(\Xi_{1}(t, s, x, \xi), \cdots, \Xi_{n}(t, s, x, \xi)\right)$ be a solution of the Cauchy problem:

$$
\left\{\begin{array}{l}
\frac{d}{d s} \Xi_{k}=A_{j, k}(t, s, x, \Xi) A_{j, 0}(t, s, x, \Xi)^{-1}(1 \leq k \leq n), \\
\left.\Xi\right|_{s=0}=\xi .
\end{array}\right.
$$

Make a change of variable via the map: $(t, s, x, \xi) \rightarrow(t, s, x, \Xi(t, s$, $x, \xi)$ ). Under the above change of variable, it is not difficult to see that $\tilde{S}^{-}[\mu, \kappa, \lambda]$ stays invariant and $H_{j, 0}$ becomes

$$
H_{j, 0}=s^{(m-1) l-1} \tilde{A}_{j, 0}(t, s, x, \Xi)\left\{s D_{s}+\tilde{B}_{j}(t, s, x, \Xi)\right\},
$$

where $\tilde{A}_{j, 0}(t, s, x, \Xi), \tilde{B}_{j}(t, s, x, \Xi)$ are the interpretation of $A_{j, 0}(t, s, x$, $\xi), B_{j}(t, s, x, \xi)$ with respect to the new variable.

Then, following the argument given in Section 5 of NakamuraUryu [13], we have the following.

Lemma 3. 12. For each $j(1 \leq j \leq m)$, there exists

$$
\tilde{a}_{m, j}^{-}(t, s, x, \xi) \in \bigcap_{\varepsilon>0} \tilde{S}^{-}\left[2 \varepsilon(\iota+1)^{-1}-(m-1), m_{j}^{+}+\varepsilon, \infty\right]
$$

such that it is flat at $s=0$ and satisfies

$$
T_{j} \tilde{a}_{m, j}^{-}+r_{m, j}^{0} \in S^{-}[-\infty] .
$$

Remark. There is a similarity between the proof of Lemma 3.12 and the following argument for constructing the compensating factors $\tilde{a}_{m, j}^{-}(1 \leq j \leq m)$. Therefore, the following argument may supplement the above short proof of Lemma 3.12.

Before we proceed to construct $\tilde{a}_{m, j}^{-}(1 \leq j \leq m)$, we have to derive 
an initial condition for each $\tilde{a}_{m, j}^{-}$. First, we estimate the effect caused by $\tilde{a}_{m, j}^{-}$. Since each term of $S_{j, h}$ is at most homogeneous of degree $h$, the additional errors caused by $\tilde{a}_{m, j}^{-}(1 \leq j \leq m)$ are $\sum_{j=1}^{m} S_{j, h} \tilde{a}_{m, j}^{-}$ $\in \tilde{S}^{-}\left[2 \varepsilon(l+1)^{-1}-(m-1)+h, \infty\right](0 \leq h \leq m-1) . \quad$ From Lemma 3. 7, there exist $\tilde{b}_{m, h}(t, x, \xi) \in S^{-}\left[2 \varepsilon(l+1)^{-1}-(m-1)+h, \infty\right](0 \leq h \leq m-1)$ such that they are flat at $t=0$ and satisfy

$$
\sum_{j=1}^{m} S_{j, h} \tilde{a}_{m, j}^{-}+\tilde{b}_{m, h} \in S^{-}[-\infty] \quad(0 \leq h \leq m-1) .
$$

On the other hand, from Lemma 3.7 and 3. 8, there exist $b_{m . h}(t, x, \xi)$ $\in \tilde{S}^{-}\left[2 \varepsilon(l+1)^{-1}-(m-1)+h, \infty\right] \quad(0 \leq h \leq m-1)$ such that they are flat at $t=0$ and satisfy

$$
\left(\sum_{j=1}^{m} S_{j, h} a_{m, j}^{-}-\delta_{h, m-1}(-1)^{m-1}\right)+b_{m, h} \in S^{-}[-\infty] .
$$

Thus, the total errors for the initial conditions are modulo $S^{-}[-\infty]$ (3.90) $\tilde{b}_{m, h}=b_{m, h}+\tilde{b}_{m, h} \in \tilde{S}^{-}\left[2 \varepsilon(l+1)^{-1}-(m-1)+h, \infty\right](0 \leq h \leq m-1)$. Therefore, the construction of the parametrix $E_{m}^{-}(t, s)$ is done if we construct $\tilde{a}_{m, j}^{-}(1 \leq j \leq m)$ which satisfy

$$
\begin{cases}T_{j} \tilde{a}_{m, j}^{-}-r_{m, j}^{1} \in S^{-}[-\infty] & (1 \leq j \leq m), \\ \sum_{j=1}^{m} S_{j, h} \tilde{a}_{m, j}^{-}-\tilde{b}_{m, h} \in S^{-}[-\infty] & (0 \leq h \leq m-1) .\end{cases}
$$

The latter condition is the one we are looking for.

We try to seek $\tilde{a}_{m, j}^{-}$as an asymptotic sum $\tilde{a}_{m, j}^{-}=\sum_{\nu=0}^{\infty} \tilde{a}_{m, j, \nu}^{-}$Following the usual integration procedure for the transport operator $T_{j}$, the conditions for $\hat{a}_{m, j, \nu}^{-}(1 \leq j \leq m, \nu \geq 0)$ are given by the following recurrence relations:

$$
\left\{\begin{array}{l}
H_{j, 0} \tilde{a}_{m, j, \nu}^{-}=-\sum_{k=1}^{\nu} H_{j, k} \tilde{a}_{m, j, \nu-k}^{-}+\delta_{0, \nu} r_{m, j}^{1} \\
\left.\tilde{a}_{m, j, \nu}\right|_{s=t}=\mathscr{V}_{j, \nu} / \mathscr{V}
\end{array}\right.
$$

where $\mathscr{V}(t, x, \xi)=\operatorname{det}\left(\left(-t^{l} \lambda_{j}(t, x, \xi)\right)^{h} ; \begin{array}{l}h \downarrow 0, \cdots, m-1 \\ j \rightarrow 1, \cdots, m\end{array}\right)$ and $\mathscr{V}_{j, \nu}(t$, $x, \xi)$ is the determinant obtained by replacing the $j$-th column of $\mathscr{V}(t, x, \xi)$ by the vector

$$
{ }^{t}\left(C_{m, \nu}^{0}(t, x, \xi), \cdots, C_{m, \nu}^{m-1}(t, x, \xi)\right) .
$$

Here, we have put

$$
C_{m, \nu}^{h}=-\left.\sum_{j=1}^{m} \sum_{k=1}^{\min (h, \nu)} M_{j, h, k} \tilde{a}_{m, j, \nu-j}^{-}\right|_{s=t}+\delta_{0, \nu} \tilde{b}_{m, h}(0 \leq h \leq m-1) .
$$


Concerning the existence of the solution $\tilde{a}_{m, j, \nu}^{-}$for (3.92), we have the following.

Lemma 3. 13. For each $j(1 \leq j \leq m)$ and $\nu \in \mathbb{Z}_{+}$, there exists $\tilde{a}_{m, j, \nu}^{-}(t, s, x, \xi) \in \bigcap_{\varepsilon>0} \widetilde{S}^{-}\left[2 \varepsilon(l+1)^{-1}-(m-1)-\nu, \infty\right]$ which satisfies (3.92).

Proof. This can be proved by taking account of $r_{m, j}^{1} \in \widetilde{S}^{-}[2 \varepsilon(l$ $\left.+1)^{-1}, \infty\right],(3.85),(3.90)$ and the following fact due to Yoshikawa [Prop. 4. 2 in [26]): Let $S_{\infty}^{-}$be the set of functions $f(t, s) \in C^{\infty}\left(\Delta_{-}\right)$ with the property: $\left|D_{t}^{k} D_{s}^{p} f(t, s)\right| \leq C|t|^{N}\left((t, s) \in \Delta_{-}, \quad k, p, N \in \mathbb{Z}_{+}\right)$ for some $C$ which depends only on $k, p, N$. Suppose $a(s), g(t)$ $\in C^{\infty}\left(\left[-T_{0}, 0\right]\right), f(t, s) \in S_{\infty}^{-}$and $g(t)$ is flat at $t=0$ then the Cauchy problem $\left(s \frac{d}{d s}+a(s)\right) u(t, s)=f(t, s), u(t, t)=g(t)$ possesses a solution $u(t, s) \in S_{\infty}^{-}$.

Q. E. D.

From Lemmas 3.7 and 3.13 , there exists $\hat{a}_{m, j}^{-}(t, s, x, \xi) \in \underset{\varepsilon>0}{\cap} \widetilde{S}^{-}[2 \varepsilon(l$ $\left.+1)^{-1}-(m-1), \infty\right]$ such that $\tilde{a}_{m, j}^{-}-\sum_{\nu<N} \tilde{a}_{m, j, \nu}^{-} \in \bigcap_{\varepsilon>0} \widetilde{S}^{-}\left[2 \varepsilon(l+1)^{-1}-(m\right.$ $-1)-N, \infty]$ for every $N \in N$. Then, consulting the proof of Lemma 3. 8, it is a routine argument to check that this $\tilde{a}_{m, j}^{-}$satisfies (3.91). This completes the construction of the parametrix $E_{m}^{-}(t, s)$.

\section{§4. Proof of Theorems 1 and 2}

First we consider Theorem 1. The existence of $a_{m, j}^{-}, \tilde{a}_{m, j}^{-}, \tilde{a}_{m, j}^{-}$ $(1 \leq j \leq m)$ which satisfy $(2.12),(2.14),(2.15)$ is clear from the argument given in Section 3, especially from Lemma 3.5, (3.64), (3.65), the properties (1), (2) of Lemma 3.8, Lemma 3.9, Lemma 3.12, (3.88), (3.89), (3.91) and the argument given after Lemma 3.13. As for the existence of $a_{i, j}^{+}, \tilde{a}_{i, j}^{+}(1 \leq i, j \leq m)$ which satisfy (2. 11), (2.13), it follows from the result of Nakamura-Uryu [13]. More precisely, the existence of $a_{i, j}^{+}, \tilde{a}_{i, j}^{+}(1 \leq i, j \leq m)$ which satisfy (2.11) follows from Theorem in P841 and the argument given in P893-894 of [13]. (2.13) can be seen by taking account of (2.12), (2.13), Lemma 2.3, Lemma 3.9, the proof of Lemma 4.2, Lemma 4.5, and the argument given in Section 5 of [13] and also (2.10) in this paper. (2.12), (2.14) and (2.15) for $i(1 \leq i \leq m-1)$ follow from the following lemma. 
Lemma 4. 1. Let

$$
q_{k-j}(s, x, \xi)=\sum_{r=0}^{k-j} p_{k-j-r, r}(s, x, \xi)(1 \leq k \leq m-1,0 \leq j \leq k-1) .
$$

Then, each $E_{m-k}^{-}(t, s)(1 \leq k \leq m-1)$ is given by

$$
E_{m-k}^{-}(t, s)=\sum_{j=0}^{k-1}\left(-D_{s}\right)^{j}\left(E_{m}^{-}(t, s) q_{k-j}\left(s, x, D_{x}\right)\right)+\left(-D_{s}\right)^{k} E_{m}^{-}(t, s) .
$$

Moreover, each $E_{i}^{-}(t, s)(1 \leq i \leq m-1)$ is written in the form (2.12) and (2.14), (2.15) are valid.

Proof. We first prove (4.2). It is enough to see this in the case each $E_{i}^{-}(t, s)(1 \leq i \leq m)$ is the fundamental solution which satisfies

$$
P E_{i}^{-}=0,\left.D_{t}^{h} E_{i}^{-}(t, s)\right|_{t=s}=\delta_{h, i-1} I(0 \leq h \leq m-1) .
$$

If we rewrite (4.3) as we did in the proof of Lemma 3.1, we find $E_{i}^{-}(t, s) \quad(1 \leq i \leq m)$ are given by

$$
\left\{\begin{array}{l}
D_{s}\left[E_{1}^{-}, \cdots, E_{m}^{-}\right]=-\left[E_{1}^{-}, \cdots, E_{m}^{-}\right] A(s), \\
{\left.\left[E_{1}^{-}, \cdots, E_{m}^{-}\right]\right|_{s=t}=[I, 0, \cdots, 0],}
\end{array}\right.
$$

where $A(s)$ is the one defined in the proof of Lemma 3.1. Then, (4.2) easily follows by writing down (4.4) explicitly. The existence of $a_{i, j}, \quad \tilde{a}_{i, j}, \quad \tilde{a}_{i, j}(1 \leq i \leq m-1,1 \leq j \leq m)$ can be seen by using the properties (9) and (10) of Lemma 3.7 and the composition formula of Fourier integral operator and pseudo-differential operator.

Finally, we prove (2.14) (2.15) for $i(1 \leq i \leq m-1,1 \leq j \leq m)$. For simplicity, we eliminate $\chi(\xi)$ from (2.12). Since the dominant symbol of $E_{m}^{-}(t, s)$ is $a_{m, q, 0}^{-, 0}(1 \leq q \leq m), a_{m-k, q, 0}^{-, 0}(1 \leq k \leq m-1,1 \leq q \leq m)$ can be derived from

$$
\sum_{j=0}^{k-1}\left(-D_{s}\right)^{j}\left(J_{m}^{-}(t, s) q_{k-j}\left(s, x, D_{x}\right)\right)+\left(-D_{s}\right)^{k} J_{m}^{-}(t, s),
$$

where

$$
\begin{gathered}
J_{m}^{-}(t, s) \cdot=(2 \pi)^{-n} \sum_{q=1}^{m} O_{s}-\iint \exp \left[\sqrt{-1}\left(\phi_{q}(t, s, x, \xi)-y \cdot \xi\right)\right] a_{m, q, 0}^{-, 0}(t, s, x, \\
\xi) \cdot d y d \xi .
\end{gathered}
$$

Using the composition formula of Fourier integral operators and pseudo-differential operators, (4.5) can be written in the form: $(2 \pi)^{-n} \sum_{q=1}^{m} O_{s}-\iint \exp \left[\sqrt{-1}\left(\phi_{q}(t, s, x, \xi)-y \cdot \xi\right)\right]\left(K_{m-k, q} a_{m, q, 0}^{-, 0}\right)(t, s, x, \xi) \cdot d y d \xi$. Then, by decomposing $K_{m-k, q}$ into semi-homogeneous parts as we did in the proof of Lemma 3.2, we can prove (2.14) and 


$$
\begin{aligned}
& a_{m-k, q, 0}^{-, 0}(t, s, x, \xi)=\exp \left[\sqrt{-1}(l+1)^{-1} s^{l+1} \lambda_{q}(0, x, \xi)\right] \\
& \quad \times M_{k}\left(\exp \left[-\sqrt{-1}(l+1)^{-1} s^{l+1} \lambda_{q}(0, x, \xi)\right] a_{m, q, 0}^{-, 0}(t, s, x, \xi)\right)
\end{aligned}
$$

where

$$
M_{k}=\Sigma^{\prime}\left(-D_{s}\right)^{j}\left(s^{(k-j-r) l-r} \tilde{P}_{k-j-r, r}(0, x, \xi) \cdot\right)+\left(-D_{s}\right)^{k}
$$

with the summation $\Sigma^{\prime}$ taken over all $j, r$ such that $0 \leq j \leq k-1,0 \leq r$ $\leq k-j,(k-j-r) l \geq r, m-k+j \geq 0$. On the other hand, reminding (2.16) holds for $i=m$ andapplying the above argument which derived (4.2) to the fundamental solution $U_{i}^{-}(1 \leq i \leq m)$ defined by (2.17), we have

$$
\begin{aligned}
U_{m-k}^{-}(t, s, x, \xi)= & \left(M_{k} U_{m}^{-}\right)(t, s, x, \xi) \\
= & \sum_{q=1}^{m} M_{k}\left[\exp \left[-\sqrt{-1}(l+1)^{-1} s^{l+1} \lambda_{q}(0, x, \xi)\right]\right. \\
& \left.\times a_{m, q, 0}^{-, 0}(t, s, x, \xi)\right] .
\end{aligned}
$$

Now, compare (2.18) and (4.8) having (4.6) in mind, then we easily see (2.15) holds for each $i(1 \leq i \leq m-1)$.

Q. E. D.

Next, we proceed to prove Theorem 2. As we mentioned right after the Remark of Theorem 1, (2.19) is clear from the composition formula for Fourier integral operators. To see (2.20), we have to look more closely about this formula.

From (2.12) we have

$$
\begin{aligned}
\left.D_{t}^{k-1} E_{i}^{-}(t, s)\right|_{t=0} \cdot= & (2 \pi)^{-n} \sum_{j=1}^{m} O_{s}-\iint \exp \left[\sqrt{-1}\left(\phi_{j}(0, s, x, \xi)-y \cdot \xi\right)\right] \\
& \times \chi(\xi) C_{i, j, k}(s, x, \xi) \cdot d y d \xi
\end{aligned}
$$

where

$$
\begin{aligned}
C_{i, j, k}(s, x, \xi) & =\exp \left[-\sqrt{-1} \phi_{j}(t, s, x, \xi)\right] \\
\times & D_{t}^{k-1}\left\{\operatorname { e x p } [ \sqrt { - 1 } \phi _ { j } ( t , s , x , \xi ) ] \cdot \left(a_{i, j}^{-}(t, s, x, \xi)\right.\right. \\
+ & \left.\left.\tilde{a}_{i, j}^{-}(t, s, x, \xi)+\tilde{a}_{i, j}^{-}(t, s, x, \xi)\right)\right\}\left.\right|_{t=0}
\end{aligned}
$$

Thus, for $t>0$, the product formula for Fourier integral operators gives us

$$
\begin{aligned}
E_{i}(t, s) \cdot & =\left.\sum_{k=1}^{m} E_{k}^{+}(t, 0) \circ\left(D_{t}^{k-1} E_{i}^{-}(t, s)\right)\right|_{t=0^{\circ}} \\
& =(2 \pi)^{-n} \sum_{\nu, \mu=1}^{m} O_{s}-\iint \exp \left[\sqrt{-1} \phi_{\nu, \mu}(t, s, x, \eta)-y \cdot \eta\right] \\
& \times a_{i, \nu, \mu}(t, s, x, \eta) \cdot d y d \eta
\end{aligned}
$$

where the principal symbol of $a_{i, \nu, \mu}(t, s, x, \eta)$ (in the classical sense) is given by 


$$
\begin{aligned}
\gamma_{\nu, \mu}( & t, s, y, \eta) \chi\left(\nabla_{x} \phi_{\nu, \mu}(t, s, x, \eta)\right) \chi(\eta) \\
& \times \sum_{k=1}^{m}\left(a_{k, \nu}^{+}\left(t, x, \nabla_{x} \phi_{\nu, \mu}(t, s, x, \eta)\right)+\tilde{a}_{k, \nu}^{+}\left(t, x, \nabla_{x} \phi_{\nu, \mu}(t, s, x, \eta)\right)\right) \\
& \times C_{i, \mu, k}\left(s, \nabla_{\eta} \phi_{\nu, \mu}(t, s, x, \eta), \eta\right)
\end{aligned}
$$

with a nonzero symbol of pseudo-differential operator $\gamma_{\nu, \mu}(t, s, y, \eta)$ of order zero which depends only on $\phi_{\nu}$ and $\phi_{\mu}$.

Now, note that we can drop $\tilde{a}_{i, j}^{-}$from (4.10), because $\tilde{a}_{\overline{i, j}}$ is flat at $t=0$. Also, since $\tilde{a}_{k, \nu}^{+}$is flat at $t=0$ and $\tilde{a}_{i, j}^{-}$is flat at $s=0$, $\tilde{a}_{k, \nu}^{+}\left(t, x, \nabla_{x} \phi_{\nu, \mu}(t, s, x, \eta)\right)$ and the part included in $C_{i, u, k}\left(s, \nabla_{\eta} \phi_{\nu, \mu}(t, s, x\right.$, $\eta), \eta)$ caused from $\tilde{a}_{i, \mu}^{-}(t, s, y, \eta)$ are very small. Thus, the main part of $a_{i, \nu, \mu}(t, s, x, \eta)$ yields from

$$
\begin{aligned}
& \gamma_{\nu, \mu}(t, s, y, \eta) \chi(\eta) \chi\left(\nabla_{x} \phi_{\nu, \mu}(t, s, x, \eta)\right) \\
& \quad \times \sum_{k=1}^{m} a_{k, \nu}^{+}\left(t, x, \nabla_{x} \phi_{\nu, \mu}(t, s, x, \eta) \widetilde{C}_{i, \mu, k}\left(s, \nabla_{\eta} \phi_{\nu, \mu}(t, s, x, \eta), \eta\right)\right.
\end{aligned}
$$

where

$$
\begin{aligned}
\widetilde{C}_{i, \mu, k}(s, y, \eta) & =\exp \left[-\sqrt{-1} \phi_{\mu}(t, s, y, \eta)\right] \\
\times & \left.D_{t}^{k-1}\left\{\exp \left[\sqrt{-1} \phi_{u}(t, s, y, \eta)\right] a_{i, \mu}^{-}(t, s, y, \eta)\right\}\right|_{t=0} .
\end{aligned}
$$

Expanding $\phi_{\mu}(t, s, y, \eta)$ into Taylor's series around $s=t=0$, the dominant part of $\tilde{C}_{i, \mu, k}(s, y, \eta)$ is

$$
\begin{aligned}
& \widetilde{C}_{i, \mu, k}(s, y, \eta)=\exp \left[-\sqrt{-1}(l+1)^{-1} t^{l+1} \lambda_{\mu}(0, y, \eta)\right] \\
& \quad \times\left. D_{t}^{k-1}\left\{\exp \left[\sqrt{-1}(l+1)^{-1} t^{l+1} \lambda_{\mu}(0, y, \eta)\right] a_{i, \mu}^{-}(t, s, y, \eta)\right\}\right|_{t=0}
\end{aligned}
$$

if $s$ is small enough. Thus reminding (2.14) and the properties (3) and (4) of Lemma 3. 7, the dominant part of $\widetilde{C}_{i, \mu, k}\left(s, \nabla_{\eta} \phi_{\nu, \mu}(t, s, x, \eta)\right.$, $\eta)$ is $C_{i, \mu, k}^{*}\left(s, \nabla_{\eta} \phi_{\nu, \mu}(t, s, x, \eta), \eta\right)$, with

$$
\begin{aligned}
& C_{i, \mu, k}^{*}(s, y, \eta)=\exp \left[-\sqrt{-1}(l+1)^{-1} t^{l+1} \lambda_{\mu}(0, y, \eta)\right] \\
& \quad \times\left. D_{t}^{k-1}\left\{\exp \left[\sqrt{-1}(l+1)^{-1} t^{l+1} \lambda_{\mu}(0, y, \eta)\right] a_{i, \mu, 0}^{-, 0}(t, s, y, \eta)\right\}\right|_{t=0}
\end{aligned}
$$

when $s, t$ are small enough and $|\eta|$ is sufficiently large. Incidentally, a similar argument as above shows that the dominant part of $a_{k, \nu}^{+}(t, x$, $\left.\nabla_{x} \phi_{\nu, \mu}(t, s, x, \eta)\right)$ is $T_{+}^{(k, \nu)}\left(x, \nabla_{x} \phi_{\nu, \mu}(t, s, x, \eta)\right) V_{\nu}^{+}\left(t, x, \nabla_{x} \phi_{\nu, \mu}(t, s, x, \eta)\right)$ when $s, t,|\eta|$ are as above. Therefore, the main part of $a_{i, \nu, \mu}(t, s$, $x, \eta)$ is

$$
\begin{aligned}
& \gamma_{\nu, \mu}(t, s, y, \eta) \chi(\eta) \chi\left(\nabla_{x} \phi_{\nu, \mu}(t, s, x, \eta)\right) \\
& \quad \times \sum_{k=1}^{m} T_{+}^{(k, \nu)}\left(x, \nabla_{x} \phi_{\nu, \mu}(t, s, x, \eta)\right) \cdot V_{\nu}^{+}\left(t, x, \nabla_{x} \phi_{\nu, \mu}(t, s, x, \eta)\right) \\
& \quad \times C_{i, \mu, k}^{*}\left(s, \nabla_{\eta} \phi_{\nu, \mu}(t, s, x, \eta), \eta\right)
\end{aligned}
$$

when $s, t$ are small enough and $|\eta|$ are sufficiently large. 
Then, (4.17) can be rewritten in the form (2.20) by the following lemma.

\section{Lemma 4. 2.}

$$
C_{i, \mu, k}^{*}(s, y, n)=\widetilde{T}_{-}^{(k, \mu)}(y, \eta) \widetilde{V}_{\mu, i-1}^{-}(s, y, \eta) \quad(1 \leq i, k, \mu \leq m) .
$$

Proof. By using (2.15), we can rewrite (4.16) in the form:

$$
C_{i, \mu, k}^{*}(s, y, \eta)=C_{\mu, k}^{*}(y, \eta) \widetilde{V}_{\mu, i-1}^{-}(s, y, \eta) \quad(1 \leq i, k, \mu \leq m) 。
$$

From (2.16), (4.16) and (4.19), we have for each $k(1 \leq k \leq m)$

$$
\begin{aligned}
& \left.D_{t}^{k-1} U_{i}^{-}(t, s, y, \eta)\right|_{t=0} \\
& \quad=\sum_{\mu=1}^{m} \exp \left[-\sqrt{-1}(l+1)^{-1} \lambda_{\mu}(0, y, \eta)\right] C_{\mu, k}^{*}(y, \eta) \widetilde{V}_{\mu, i-1}^{-}(s, y, \eta) .
\end{aligned}
$$

On the other hand, from (2.18), we have for each $k(1 \leq k \leq m)$

$$
\begin{aligned}
& \left.D_{t}^{k-1} U_{i}^{-}(t, s, y, \eta)\right|_{t=0} \\
& \quad=\sum_{\mu=1}^{m} \exp \left[-\sqrt{-1}(l+1)^{-1} s^{l+1} \lambda_{\mu}(0, y, \eta)\right] \widetilde{T}_{-}^{(k, \mu)}(y, \eta) \widetilde{V}_{\mu, i-1}^{-}(s, y, \eta) .
\end{aligned}
$$

Then, reminding $\lambda_{\mu}(0, y, \eta)(1 \leq \mu \leq m)$ are distinct and each $\widetilde{V}_{\mu, i-1}^{-}(s$, $y, \eta)(1 \leq \mu \leq m)$ admits an asymptotic expansion as $s$ tends to $-\infty$, the argument given in P871 of Uryu Nakamura [13] entails $C_{\mu, k}^{*}(y$, $\eta)=\widetilde{T}_{-}^{(k, \mu)}(y, \eta)(1 \leq k, \mu \leq m)$. Hence, by (4.19), we obtain (4.18).

Q. E. D.

Finally, if we remind that our operator $P$ is strictly hyperbolic for $t \neq 0$, the assertion (2) of Theorem 2 follows by combining the following lemma with the well known result about the propagation of singularities for strictly hyperbolic operators.

Lemma 4. 3. Let $i(0 \leq i \leq m-1)$ be an integer and $v_{h} \in \mathscr{E}^{\prime}\left(\mathbb{R}^{n}\right)$ $(0 \leq h \leq m-1)$ such that $\bigcup_{h \neq i} W F\left(v_{h}\right)=\phi, W F\left(v_{i}\right)=\left\{\left(y^{0}, \rho \eta^{0}\right) ; \rho>0\right\}$. Moreover, let $v$ be the solution of the Cauchy problem: Pv $=0$, $D_{t}^{h} v !_{l=s^{\prime}}=v_{h}(0 \leq h \leq m-1)$. Then, under the condition (\#), we have

$$
T_{\nu_{0}, \mu_{0}}\left(t^{\prime}, s^{\prime}\right)\left(y^{0}, \eta^{0}\right) \in W F\left(v\left(t^{\prime}, s^{\prime}\right)\right) \text {. }
$$

Proof. Reminding the assertion (1) of Theorem 2, this can be proved by a routine argument. Hence, we only point out the following facts (i) and (ii) which are necessary for the proof. 
(i ) $V_{i}^{ \pm}(t, x, \xi)(1 \leq i \leq m)$ are semi-homogeneous of degree zero, and are nonvanishing for $\pm t>0$ and sufficiently large $|\xi|$.

(ii) Each $T_{ \pm}^{(i, j)}(x, \xi) \quad(1 \leq i, j \leq m)$ is semi-homogeneous of degree $-(l+1)^{-1}(i-1)$. Consequently, the condition (\#) implies

$$
\sum_{j=1}^{m} T_{+}^{\left(j, \nu_{0}\right)}\left(T_{\nu_{0}, \mu_{0}}\left(t^{\prime}, s^{\prime}\right)(y, \eta)\right) \tilde{T}_{-}^{\left(j, \mu_{0}\right)}(y, \eta) \neq 0
$$

in a conic neighborhood of $\left(y^{0}, \eta^{0}\right)$.

(i) can be seen from the argument given in P206-207 of Amano [2]. (ii) follows from the relation (2.10) reminding (i) and each $V_{i}(t, x, \xi)(1 \leq i \leq m)$ is semi-homogeneous of degree $-(l+1)^{-1}(i-1)$.

Q. E. D.

\section{§. Proof of Theorem 3 and Some Discussions}

In this section we proceed as follows. We first show the procedure how to calculate $T_{+}^{(i, j)}(x, \xi)(1 \leq i, j \leq 2)$. And then slightly modifying this procedure, we calculate $T_{-}^{(i, j)}(x, \xi)(1 \leq i, j \leq 2)$. Finally, we compare our results to the others.

In the case $m=2, L_{0}$ is

$$
L_{0}=D_{t}^{2}+t^{l} \tilde{P}_{1,0}(0, x, \tilde{\varsigma}) D_{t}+t^{l-1} \tilde{P}_{1,1}(0, x, \xi)+t^{2 l} \tilde{P}_{2,0}(0, x, \xi) \text {. }
$$

For simplicity we omit the variable $x$ and the value zero in the rest of the argument. For example, we write $\tilde{P}_{1,0}(\xi), \lambda_{1}(\xi)$ instead of $\tilde{P}_{1,0}(0, x, \xi), \lambda_{1}(0, x, \xi)$. Under this convention, $L_{0}$ is

$$
L_{0}=D_{t}^{2}+t^{l} \tilde{P}_{1,0}(\xi) D_{t}+t^{l-1} \tilde{P}_{1,1}(\xi)+t^{2 l} \tilde{P}_{2,0}(\xi) \text {. }
$$

Introducing a new variable $z=(l+1)^{-1} t^{l+1}|\xi|, L_{0}$ becomes

$$
L_{0}=-t^{2 l}|\xi|^{2} \widetilde{L}_{0}
$$

where

$$
\begin{aligned}
\tilde{L}_{0}=\partial_{z}^{2} & +\left(\sqrt{-1} \tilde{P}_{1,0}(\theta)+l(l+1)^{-1} z^{-1}\right) \partial_{z} \\
& -\left(\tilde{P}_{2,0}(\theta)+(l+1)^{-1} z^{-1} \tilde{P}_{1,1}(\theta)\right)
\end{aligned}
$$

with $\partial_{z}=\partial / \partial z, \theta=\xi /|\xi|$.

(5.4) is the so-called confluent type equation. The global theory for this type is shown in p 249-280 of Hukuhara's book [8]. Unfortunately, it is written in Japanese. Since we could not find any other accessible document which deals with this subject, we dare illustrate some of its part briefly to help the reader's understanding. Let's 
start by stating the precise definition of confluent type equation and other related notations and definitions which are necessary for the later arguments.

Notation. (i) $\overrightarrow{\zeta_{1} \zeta_{2}}$ denotes the straight path starting from point $\zeta_{1} \in \mathbb{C}$ to $\zeta_{2} \in C$. (ii) $\left\langle\zeta_{0},(\omega)\right\rangle$ denotes the path: $\zeta_{0}+r \exp [\sqrt{-1} \omega]$ $(0 \leq r<\infty)$. (iii) $\int_{\zeta_{0}}^{(\omega)} w(\zeta) d \zeta$ denotes the path integral of $w(\zeta)$ along the path $\left\langle\zeta_{0},(\omega)\right\rangle$. (iv) $A\left\langle\omega_{1}, \omega_{2}\right\rangle$ denotes the sector $\{z \in \mathbb{C}-\{0\}$; $\left.\omega_{1}<\arg z<\omega_{2}\right\}$. (v) We also use the usual symbol of integral to denote the finite part of divergent integral.

Definition. The confluent type equation is an equation which has the following form:

$$
z v^{\prime \prime}(z)+\left(a_{1} z+b_{1}\right) v^{\prime}(z)+\left(a_{2} z+b_{2}\right) v(z)=0,
$$

where $a_{i}, b_{i}(i=1,2)$ are constants. (5.5) may have a regular singular point at $z=0$ and an irregular singular point of Poincaré's rank one at $z=\infty$, but there are no other singular points. Let $\delta_{1}, \delta_{2}$ be the roots of the indicial equation of (5.5) at $z=0$, and let each $\alpha_{i}(i=1,2)$ and $\kappa_{i}(i=1,2)$ be the root of the equation

$$
\begin{aligned}
& \rho^{2}+a_{1} \rho+a_{2}=0 \text { and } \\
& \left(2 \alpha_{i}+a_{1}\right) \kappa_{i}-b_{1} \alpha_{i}-b_{2}=0
\end{aligned}
$$

respectively. For the sake of later reference, we temporarily call each $\delta_{i}, \alpha_{i}, \kappa_{i}(i=1,2)$ indicial root, characteristic root, characteristic exponent respectivelyin this paper. Also, we denote the general solution of $(5.5)$ by

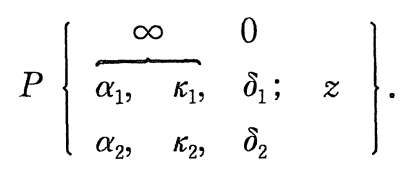

Remark. (1) It is known that the extended Fuch's relation: $\delta_{1}+\delta_{2}+\kappa_{1}+\kappa_{2}=1$ holds provided $\alpha_{1} \neq \alpha_{2}$. Conversely, given $\alpha_{i}, \kappa_{i}, \delta_{i}$ $(i=1,2)$ which satisfy $\alpha_{1} \neq \alpha_{2}$ and the extended Fuch's relation, the equation (5.5) is uniquely determined by these six quantities.

(2) Let $v(z)=\int_{C} \exp [-z \zeta] w(\zeta) d \zeta$ be the Laplace transform of $w(\zeta)$, where the contour $C$ is taken appropriately. Then $v(z)$ will 
be a solution of $(5.5)$ if $w(\zeta)$ satisfies

$$
\left(\zeta^{2}-a_{1} \zeta+a_{2}\right) w^{\prime}(\zeta)+\left\{\left(2-b_{1}\right) \zeta+b_{2}-a_{1}\right\} w(\zeta)=0 .
$$

Now, set

$$
\gamma_{i}=\kappa_{i}-1(i=1,2) .
$$

Then, it is easy to see

$$
\phi(\zeta)=\left(\zeta+\alpha_{1}\right)^{\gamma_{1}}\left(\zeta+\alpha_{2}\right)^{\gamma_{2}}
$$

is a particular solution of (5.8).

(3) There are two types of canonical fundamental systems of solutions for (5.5) which describe the behavior around the singular points $z=0$ and $z=\infty$ respectively. The problem of finding the explicit formula of the linear relation between these two fundamental systems of solutions is called the two point connection problem or central connection problem. The explicit formula of $T_{ \pm}^{(i, j)}(x, \xi)(i, j$ $=1,2$ ) can be obtained by solving the central connection problem.

Reminding the relations $\tilde{P}_{1,0}(\theta)=-\left(\lambda_{1}(\theta)+\lambda_{2}(\theta)\right)$ and $\tilde{P}_{2,0}(\theta)$ $=\lambda_{1}(\theta) \lambda_{2}(\theta)$, we easily obtain that the general solution for the operator (5. 4) is

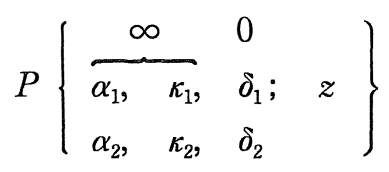

with

$$
\begin{aligned}
& \alpha_{i}=\sqrt{-1} \lambda_{i}(\theta), \\
& \kappa_{i}=(-1)^{i-1}(l+1)^{-1}\left(\lambda_{1}(\theta)-\lambda_{2}(\theta)\right)^{-1}\left(l \lambda_{i}(\theta)+\sqrt{-1} \tilde{P}_{1,1}(\theta)\right) . \\
& \delta_{i}=\delta_{i, 2}(l+1)^{-1}(i=1,2) .
\end{aligned}
$$

The following lemma is not a complete answer to the central connection problem for the confluent type equation (5.5), but it is enough to obtain the explicit formula of $T_{ \pm}^{(i, j)}(x, \xi)(i, j=1,2)$.

Lemma 5. 1. Assume $\kappa_{i}, \kappa_{i}+\delta_{2} \notin \mathbb{Z}(i=1,2),\left|\tau^{0}\right|<\pi / 2$. Set

$$
\begin{gathered}
\text { (5. 13) } \quad \phi_{i}(z)=\Gamma\left(\gamma_{i}+1\right)^{-1}\left\{(-1)^{i-1}\left(\alpha_{2}-\alpha_{1}\right)\right\}^{-\gamma_{i}} \phi_{i}^{*}(z), \\
\text { (5. 14) } \quad \phi_{i}^{*}(z)=\int_{-\alpha_{i}}^{(\tau)} \exp [-z \zeta] \psi(\zeta) d \zeta\left(z \in A\left\langle-\tau^{0}-\pi / 2,-\tau^{0}+\pi / 2\right\rangle\right),
\end{gathered}
$$

where $|\tau+\arg z|<\frac{\pi}{2}$. Let each $\hat{\phi}_{i}(z)(i=1,2)$ be a solution of 
with an expansion:

$$
\hat{\phi}_{i}(z)=z^{\delta_{i}}\left(1+\sum_{r=1}^{\infty} d_{i, r} z^{r}\right) .
$$

Moreover, we assume $\alpha_{1}, \alpha_{2}$ are purely imaginary. Then, the following assertions hold.

(1) Each $\phi_{i}(z)(i=1,2)$ admits an asymptotic expansion:

$$
\phi_{i}(z) \simeq \exp \left[\alpha_{i} z\right] z^{-\kappa_{i}}\left(1+\sum_{r=1}^{\infty} B_{i, r} z^{-r}\right)
$$

as $z \rightarrow \infty$ in the sector $A\left\langle-\tau^{0}-\pi / 2,-\tau^{0}+\pi / 2\right\rangle$.

(2) If $\sqrt{-1} \alpha_{2}>\sqrt{-1} \alpha_{1}$, we have

$$
\hat{\phi}_{i}(z)=\sum_{j=1}^{2} C_{i, j} \phi_{j}(z) \quad(z \neq 0 ; i=1,2)
$$

with

$$
\left\{\begin{aligned}
C_{1,1} & =\Gamma\left(\delta_{2}\right)^{-1} \Gamma\left(\gamma_{1}+\delta_{2}+1\right)\left(\alpha_{2}-\alpha_{1}\right)^{\gamma_{2}+\delta_{2}} \\
& \times\left(1-\exp \left[-\pi \sqrt{-1}\left(\gamma_{1}+\delta_{2}\right)\right] \sin \pi\left(\gamma_{2}+\delta_{2}\right) / \sin \pi\left(\gamma_{1}+\gamma_{2}+2 \delta_{2}\right)\right) \\
C_{1,2} & =\Gamma\left(\delta_{2}\right)^{-1} \Gamma\left(\gamma_{2}+\delta_{2}+1\right)\left(\alpha_{1}-\alpha_{2}\right)^{\gamma_{1}+\delta_{2}} \\
& \times \exp \left[-\pi \sqrt{-1}\left(\gamma_{1}+\delta_{2}\right)\right] \sin \pi\left(\gamma_{2}+\delta_{2}\right) / \sin \pi\left(\gamma_{1}+\gamma_{2}+2 \delta_{2}\right) \\
C_{2,1} & =\Gamma\left(-\delta_{2}\right)^{-1} \Gamma\left(\gamma_{1}+1\right)\left(\alpha_{2}-\alpha_{1}\right)^{\gamma_{2}} \\
& \times\left(1-\exp \left[-\pi \sqrt{-1} \gamma_{1}\right] \sin \pi \gamma_{2} / \sin \pi\left(\gamma_{1}+\gamma_{2}\right)\right) \\
C_{2,2} & =\Gamma\left(-\delta_{2}\right)^{-1} \Gamma\left(\gamma_{2}+1\right)\left(\alpha_{1}-\alpha_{2}\right)^{\gamma_{1}} \\
& \times \exp \left[-\pi \sqrt{-1} \gamma_{1}\right] \sin \pi \gamma_{2} / \sin \pi\left(\gamma_{1}+\gamma_{2}\right)
\end{aligned}\right.
$$

(3) If $\sqrt{-1} \alpha_{1}>\sqrt{-1} \alpha_{2}$, (5.17) holds with

$$
\left\{\begin{aligned}
C_{1,1} & =\Gamma\left(\delta_{2}\right)^{-1} \Gamma\left(\gamma_{1}+\delta_{2}+1\right)\left(\alpha_{2}-\alpha_{1}\right)^{\gamma_{2}+\delta_{2}} \\
& \times \exp \left[-\pi \sqrt{-1}\left(\gamma_{2}+\delta_{2}\right)\right] \sin \pi\left(\gamma_{1}+\delta_{2}\right) / \sin \pi\left(\gamma_{1}+\gamma_{2}+2 \delta_{2}\right), \\
C_{1,2} & =\Gamma\left(\delta_{2}\right)^{-1} \Gamma\left(\gamma_{2}+\delta_{2}+1\right)\left(\alpha_{1}-\alpha_{2}\right)^{\gamma_{1}+\delta_{2}} \\
& \times\left(1-\exp \left[-\pi \sqrt{-1}\left(\gamma_{2}+\delta_{2}\right)\right] \sin \pi\left(\gamma_{1}+\delta_{2}\right) / \sin \pi\left(\gamma_{1}+\gamma_{2}+2 \delta_{2}\right)\right), \\
C_{2,1} & =\Gamma\left(-\delta_{2}\right)^{-1} \Gamma\left(\gamma_{1}+1\right)\left(a_{2}-\alpha_{1}\right)^{\gamma_{2}} \\
& \times \exp \left[-\pi \sqrt{-1} \gamma_{2}\right] \sin \pi \gamma_{1} / \sin \pi\left(\gamma_{1}+\gamma_{2}\right) \\
C_{2,2} & =\Gamma\left(-\delta_{2}\right)^{-1} \Gamma\left(\gamma_{2}+1\right)\left(\alpha_{1}-\alpha_{2}\right)^{\gamma_{1}} \\
& \times\left(1-\exp \left[-\pi \sqrt{-1} \gamma_{2}\right] \sin \pi \gamma_{1} / \sin \pi\left(\gamma_{1}+\gamma_{2}\right)\right) .
\end{aligned}\right.
$$

Proof. (1) follows from the well known asymptotic property of Laplace integral. Since (3) can be proved in the same as (2), we only prove (2). Let $\Phi_{1}(z)=\int_{-\alpha_{1}}^{\alpha_{2}} \phi(\zeta) d \zeta$ and $\hat{\phi}(z)$ be a solution of equation (5.5) with an expansion 


$$
\hat{\phi}_{2}(z)=z^{\delta_{2}} \sum_{r=0}^{\infty} \hat{d}_{2, r} z^{r}
$$

near $z=0$. Since the pair $\phi_{1}^{*}(z)$ and $\Phi_{1}(z)$ is a fundamental system of solution of (5.5), the relation

$$
\phi_{1}^{*}(z)=\hat{\phi}_{2}(z)+K_{1} \Phi_{1}(z)
$$

holds for some constant $K_{1}$. We want to compute $K_{1}$ and $\hat{d}_{2,0}$. To compute $K_{1}$, we continuate the both side of (5.21) analytically around $z=0$. For instance, take a sufficiently large $R>0$ and vary $z$ along $|z|=R$ counterclockwise starting from $R \exp \left[-\sqrt{-1} \tau^{0}\right]$. As $z$ varies from $R \exp \left[-\sqrt{-1} \tau^{0}\right]$ to $R \exp \left[\sqrt{-1}\left(-\tau^{0}+\pi\right)\right]$, we vary $\tau$ to satisfy the relation

$$
\text { arg } z+\tau=0 \text {. }
$$

When $z$ reaches the point $R \exp \left[\sqrt{-1}\left(-\tau^{0}+\pi\right)\right]$, using Cauchy's integration theorem, we replace $\int_{-\alpha_{1}}^{\left(\tau^{0}-\pi\right)} \exp [-z \zeta] \psi(\zeta) d \zeta$ by $\left(\int_{-\alpha_{1},-\alpha_{2}}+\right.$ $\left.\int_{-\alpha_{2}}^{\left(\tau^{0}-\pi\right)}\right) \exp [-z \zeta] \psi(\zeta) d \zeta$. After that we proceed the analytic continuation along $|z|=R$ from $R \exp \left[\sqrt{-1}\left(-\tau^{0}+\pi\right)\right]$ to $R \exp \left[\sqrt{-1}\left(-\tau^{0}\right.\right.$ $+2 \pi)]$ letting $\tau$ to satisfy (5.22). Then, at the time $z$ reaches the point $R \exp \left[-\sqrt{-1} \tau^{0}\right], \int_{-\alpha_{2}}^{\left(\tau^{0}-\pi\right)} \exp [-z \zeta] \psi(\zeta) d \zeta$ becomes

$$
\int_{-\alpha_{2}}^{\left(\tau^{0}-2 \pi\right)} \exp [-z \zeta] \psi(\zeta) d \zeta
$$

By Cauchy's integration theorem, (5.23) is equal to

$$
\left(\int_{-\alpha_{2},-\alpha_{1}}+\int_{-\alpha_{1}}^{\left(\tau^{0}-2 \pi\right)}\right) \exp [-z \zeta] \psi(\zeta) d \zeta .
$$

Thus, when $z$ comes back to the starting point, the integral

$$
\phi_{1}^{*}(z)=\int_{-\alpha_{1}}^{\left(\tau^{0}\right)} \exp [-z \zeta] \psi(\zeta) d \zeta
$$

is the sum of integration along the paths $\overrightarrow{-\alpha_{1},-\alpha_{2}}, \overrightarrow{-\alpha_{2},-\alpha_{1}},<-\alpha_{1}$, $\left.\left(\tau^{0}-2 \pi\right)\right\rangle$. Therefore, taking account of the change of the integrands, especially those arguments, and the fact that the respective change of $\hat{\phi}_{2}(z)$ and $\Phi_{1}(z)$ after the analytic continuation are $\exp \left[2 \pi \sqrt{-1} \delta_{2}\right] \hat{\phi}_{2}(z)$ and $\Phi_{1}(z),(5.21)$ implies

$$
\begin{aligned}
& \exp \left[2 \pi \sqrt{-1} \delta_{2}\right] \hat{\phi}_{2}(z)+K_{1} \Phi_{1}(z) \\
& \quad=\exp \left[-2 \pi \sqrt{-1}\left(\gamma_{1}+\gamma_{2}\right)\right]\left(\hat{\phi}_{2}(z)+K_{1} \Phi_{1}(z)\right)
\end{aligned}
$$




$$
+\left(\exp \left[-2 \pi \sqrt{-1} \gamma_{1}\right]-\exp \left[-2 \pi \sqrt{-1}\left(\gamma_{1}+\gamma_{2}\right)\right]\right) \Phi_{1}(z) \text { 。 }
$$

In order that (5.24) is valid, we have the following condition:

$$
\left\{\begin{array}{l}
\left(1-\exp \left[-2 \pi \sqrt{-1}\left(\gamma_{1}+\gamma_{2}\right)\right]\right) K_{1} \\
=\exp \left[-2 \pi \sqrt{-1} \gamma_{1}\right]-\exp \left[-2 \pi \sqrt{-1}\left(\gamma_{1}+\gamma_{2}\right)\right] \\
\exp \left[2 \pi \sqrt{-1} \delta_{2}\right]=\exp \left[-2 \pi \sqrt{-1}\left(\gamma_{1}+\gamma_{2}\right)\right] .
\end{array}\right.
$$

Here, the second condition is valid from the extended Fuch's relation. Thus, solving $K_{1}$ from the first condition of (5.25), we obtain

$$
K_{1}=\exp \left[-\pi \sqrt{-1} \gamma_{1}\right] \sin \pi \gamma_{2} / \sin \pi\left(\gamma_{1}+\gamma_{2}\right) \text {. }
$$

Note that, by using the relation $\Phi_{1}(z)=\phi_{1}^{*}(z)-\phi_{2}^{*}(z)$, we can convert (5.20) into the form:

$$
\hat{\phi}_{2}(z)=\left(1-K_{1}\right) \phi_{1}^{*}(z)+K_{1} \phi_{2}^{*}(z) \text {. }
$$

Next we compute $\hat{d}_{2,0}$. Since $\phi(\zeta)=\sum_{r=0}^{\infty} e_{r}\left(\zeta+\alpha_{2}\right)^{r_{1}+r_{2}-r}$ with $e_{0}=1$, the well known asymptotic property of Laplace integral entails

$$
\hat{\phi}_{2}(z)=\exp \left[\alpha_{1} z\right] \sum_{r=0}^{\infty} e_{r} \Gamma\left(-\delta_{2}-r\right) z^{\delta_{2}+r}
$$

by taking account of the fact $\gamma_{1}+\gamma_{2}=-\left(\delta_{2}+1\right) \notin \mathbb{Z}$ and (5.20). Comparing (5.20) and (5.28), we obtain

$$
\hat{d}_{2,0}=\Gamma\left(-\delta_{2}\right) \text {. }
$$

Hence (5.17) for $i=2$ and $C_{2, j}(j=1,2)$ of (5.18) follows immediately from (5.13), (5.20), (5.26), (5.27) and (5.29).

The rest of the proof can be done by using the simple fact:

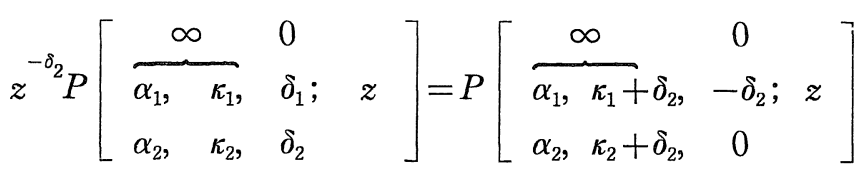

Q. E. D.

Now, we proceed to prove Theorem 3. Since the procedures are similar, we only consider the case $\lambda_{1}(0, x, \xi)>\lambda_{2}(0, x, \xi)$. We first calculate $T_{+}^{(i, j)}(x, \xi)(i, j=1,2)$. Reminding (2.9), (5.3), (5.11), (5.13) and (5.15), we easily obtain

$$
\left\{\begin{array}{l}
U_{1}(t, x, \xi)=\hat{\phi}_{1}\left((l+1)^{-1} t^{l+1}|\xi|\right), \\
U_{2}(t, x, \xi)=\sqrt{-1}(l+1)^{(l+1)^{-1}}|\xi|^{-(l+1)^{-1}} \hat{\phi}_{2}\left((l+1)^{-1} t^{l+1}|\xi|\right) .
\end{array}\right.
$$

Also, from (2.3), (2.4), (2.6), (5.3) and (5.16), we have 


$$
\begin{aligned}
& \exp \left[\sqrt{-1}(l+1)^{-1} t^{l+1} \lambda_{i}(0, x, \xi)\right] V_{i}^{+}(t, x, \xi) \\
& =(l+1)^{-\kappa_{i}} \phi_{i}\left((l+1)^{-1} t^{l+1}|\xi|\right)(i=1,2) .
\end{aligned}
$$

Then, the assertion (1.i) of Theorem 3 follows from the assertion (2) of Lemma 5.1, (5.30) and (5.31).

Next, we skip to prove the assertion (3.i) of Theorem 3, because the assertion (2) can be proved much easier. Set

$$
L_{1}=D_{t}^{2}-t^{l} \tilde{P}_{1,0}(\xi) D_{t}-t^{l-1} \tilde{P}_{1,1}(\xi)+t^{2 l} \tilde{P}_{2,0}(\xi)
$$

and let $v(t, \xi)$ be a solution of $L_{1} v=0$. Then, it is easy to see

$$
u(t, \xi)=v(-t, \xi)
$$

satisfies $L_{0} u=0$. In terms of the variable $z=(l+1)^{-1} t^{l+1}|\xi|, L_{1}$ becomes

$$
L_{1}=-t^{2 l}|\xi|^{2} \widetilde{L}_{1}
$$

with

$$
\begin{aligned}
\widetilde{L}_{1}=\partial_{z}^{2}+\left\{-\sqrt{-1} \tilde{P}_{1,0}(\theta)\right. & \left.+l(l+1)^{-1} z^{-1}\right\} \partial_{z} \\
& -\left\{\tilde{P}_{2,0}(\theta)-(l+1)^{-1} z^{-1} \tilde{P}_{1,1}(\theta)\right\}
\end{aligned}
$$

Calculating the indicial roots $\tilde{\delta}_{i}(i=1,2)$, characteristic roots $\tilde{\alpha}_{i}(i=1,2)$ and characteristic exponents $\tilde{\kappa}_{i}(i=1,2)$ for $\tilde{L}_{1}$, the general solution for the operator $\tilde{L}_{1}$ is

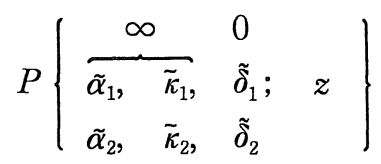

with

$$
\tilde{\alpha}_{i}=-\alpha_{i}, \tilde{\kappa}_{i}=\kappa_{i}, \quad \tilde{\delta}_{i}=\delta_{i}(i=1,2),
$$

where $\alpha_{i}, \kappa_{i}, \delta_{i}(i=1,2)$ are given by (5.12).

Now, define each $J_{i}(t, x, \xi), K_{i}^{+}(t, x, \xi), G_{+}^{(i, j)}(x, \xi)(i, j=1,2)$ by the following relations:

$$
\begin{aligned}
& L_{1} J_{i}=0,\left.D_{t}^{h} J_{i}\right|_{t=0}=\delta_{h, i-1} \quad(h=0,1), \\
& \left\{\begin{array}{l}
L_{1}\left(\exp \left[(l+1)^{-1} t^{l+1} \tilde{\alpha}_{i}\right] K_{i}^{+}\right)=0, \\
K_{i}^{+}(t, x, \xi) \simeq t^{-R_{i}(l+1)}\left(1+\sum_{r=1}^{\infty} g_{i, r}(x, \xi) t^{-r}\right) \quad(t \rightarrow \infty), \\
J_{i}(t, x, \xi)=\sum_{j=1}^{2} \exp \left[(l+1)^{-1} t^{l+1} \tilde{\alpha}_{j}\right] G_{+}^{(i, j)}(x, \xi) K_{j}^{+}(t, x, \xi)
\end{array}\right.
\end{aligned}
$$

Then, taking account of $\sqrt{-1} \tilde{\alpha}_{1}>\sqrt{-1} \tilde{\alpha}_{2}$, we can calculate $G_{+}^{(i, j)}(x, \xi)$ 
$(i, j=1,2)$ explicitly by applying the assertion (3) of Lemma 5.1. Furthermore, it is easy to prove the following:

$$
\left\{\begin{array}{l}
U_{i}(t, x, \xi)=(-1)^{i-1} J_{i}(-t, x, \xi), \\
V_{i}^{-}(t, x, \xi)=(-1)^{\kappa_{i}(l+1)} K_{i}^{+}(-t, x, \xi)
\end{array}\right.
$$

for $t \leq 0, i=1,2$. By $(5.40)$ and

$$
\exp \left[(l+1)^{-1}(-t)^{l+1} \tilde{\alpha}_{i}\right]=\exp \left[\sqrt{-1}(l+1)^{-1} t^{l+1} \lambda_{i}(0, x, \xi)\right](i=1,2),
$$

we can convert the relation (5.39) into the linear relation between $U_{i}(t, x, \xi)(i=1,2)$ and

$$
\exp \left[\sqrt{-1}(l+1)^{-1} t^{l+1} \lambda_{i}(0, x, \xi)\right] V_{i}^{-}(t, x, \xi)(i=1,2) .
$$

Then, observing this relation, we obtain

$$
T_{-}^{(i, j)}(x, \xi)=(-1)^{-\kappa_{j}(l+1)+i-1} G_{\perp}^{(i, j)}(x, \xi) \quad(i, j=1,2) .
$$

The assertion (3. i) immediately follows from (5.41). Thus we have proved Theorem 3.

Now, take an operator $P=D_{t}^{2}-t^{2 l} D_{x}^{2}+\sqrt{-1} a t^{l-1} D_{x}$, where $x \in \mathbb{R}^{1}$, $l \in N$, and compare the results of ours with that of Hanges [7] and Taniguchi-Tozaki [22] for this operator. Set $\tilde{\lambda}_{i}(t, x, \xi)=(-1)^{i-1} t^{l} \xi$, $\lambda_{i}(t, x, \xi)=(-1)^{i-1} \xi(i=1,2)$ and denote the subprincipal symbol of $P_{1}-(2 \sqrt{-1})^{-1}\left(\partial_{t} \partial_{\tau} P_{2}+\partial_{x} \partial_{\xi} P_{2}\right)$ by $S_{p}(t, x, \tau, \xi)$, where each $P_{i}(i=1,2)$ is the homogeneous part of degree $i$ of $P$. Given $i(i=0,1)$ and $u_{h} \in \mathscr{E}^{\prime}\left(\boldsymbol{R}^{n}\right)(h=0,1)$ such that $\bigcup_{h \neq i} W F\left(u_{h}\right)=\phi, W F\left(u_{i}\right)=\left\{\left(y^{0}, \rho \eta^{0}\right)\right.$; $\rho>0\}$, let $u(t, x)$ be a solution of the Cauchy problem: $P u=0,\left.D_{t}^{h} u\right|_{t=-1}$ $=u_{h}(h=0,1)$.

When $l=1, P$ is doubly noninvolutive characteristic at $t=0$. Hence, the Hanges' result [7] is applicable.

Theorem A. (Hanges [7]) Let $l=1$. Then, under the condition

$$
\begin{aligned}
(-1)^{k} \sqrt{-1} S_{p} /\left\{\tau-\tilde{\lambda}_{1}, \tau-\tilde{\lambda}_{2}\right\}-\frac{1}{2} \notin Z_{+} \\
\text {at } t=\tau=0, \quad(x, \xi)=T_{k}(0,-1)\left(y^{0}, \eta^{0}\right),
\end{aligned}
$$

we have $T_{j, k}(t,-1)\left(y^{0}, \eta^{0}\right) \in W F(u(t, \cdot))$ for any $j(j=1,2)$ and $t>0$.

For general $l \in N$, there is the following result due to Taniguchi and Tozaki. 
Theorem B. (Taniguchi-Tozaki [22]).

(1) $W F(u(t, \circ))=\bigcup_{j=1}^{2}\left\{T_{j}(t,-1)\left(y^{0}, \eta^{0}\right)\right\} \cup\left\{T_{1,2}(t,-1)\left(y^{0}, \eta^{0}\right)\right\}(t>0)$ when $a=2 n(l+1)+l+m \quad\left(n \in \mathbb{Z}_{+}, m=0,2\right)$.

(2) $W F(u(t, \cdot))=\bigcup_{j=1}^{2}\left\{T_{j}(t,-1)\left(y^{0}, \eta^{0}\right)\right\} \cup\left\{T_{2,1}(t,-1)\left(y^{0}, \eta^{0}\right)\right\}(t>0)$ when $a=-[2 n(l+1)+l+m]\left(n \in \mathbb{Z}_{+}, \quad m=0,2\right)$.

(3) (This type occurs only when $l$ is even).

$$
W F(u(t, \circ))=\bigcup_{j \neq k}\left\{T_{j, k}(t,-1)\left(y^{0}, \eta^{0}\right)\right\}(t>0)
$$

when $a=(l+1)(1 \pm 2 n)\left(n \in \mathbb{Z}_{+}\right)$.

(4) $\mathrm{WF}(u(t, \cdot))=\bigcup_{j=1}^{2}\left\{T_{j}(t,-1)\left(y^{0}, \eta^{0}\right)\right\} \cup \bigcup_{j \neq k}\left\{T_{j, k}(t,-1)\left(y^{0}, \eta^{0}\right)\right\}(t>0)$ in the other cases.

Remark. It is easy to see the condition (5.42) is equivalent to

$$
a \neq(-1)^{k-1}(2 n+1)\left(n \in \mathbb{Z}_{+}\right) .
$$

On the other hand, we easily see

(5.44) $\left\{2 n(l+1)+l+m ; n \in \mathbb{Z}_{+}, m=0,2\right\}=\left\{2 n+1 ; n \in \mathbb{Z}_{+}\right\}$

when $l=1$. Thus, by $(5.43)$ and $(5.44)$, it is clear that Theorem $A$ follows from Theorem $B$.

Now, let's look at our results. Since the Fourier transform of $P$ with respect to $x$ itself is equal to our operator $L_{0},(2.13),(2.14)$ and (2.15) are identically zero. Also, we note that $a_{i, j, 0}^{ \pm, 0}(i, j=1,2)$ are independent of $x$ and each phase $\phi_{i}(t, s, x, \xi)(i=1,2)$ has a very simple form:

$$
\phi_{i}(t, s, x, \xi)=x \xi+(-1)^{i-1}(l+1)^{-1}\left(t^{l+1}-s^{l+1}\right) \xi .
$$

Furthermore, the composition formula for Fourier integral operators with these phases $\phi_{i}$ and amplitudes $a_{i, j, 0}^{ \pm, 0}$ is very simple and it can be carried out by Fourier inversion formula. Hence, if we apply this formula in the construction of $E_{m}(t, \xi)$, we can revise (2. 20). Namely,

$$
a_{i, \nu, \mu}(t, s, x, \eta)=\chi(\eta)^{2} \sum_{j=1}^{m} T_{+}^{(j, \nu)}(\eta) \tilde{T}_{-}^{(j, \mu)}(\eta) V_{\nu}^{+}(t, \eta) \widetilde{V}_{\mu, i-1}(s, \eta) .
$$

Taking account of (5.45), we have the following revised version of Theorem 2.

Theorem C. For a particular pair $\left(\nu_{0}, \mu_{0}\right)$, the necessary and 
sufficient condition for

$$
T_{\nu_{0}, \mu_{0}}(t,-1)\left(y^{0}, \eta^{0}\right) \in W F(u(t, \cdot)) \quad(t>0)
$$

is

$$
\sum_{j=1}^{2} T_{+}^{\left(j, \nu_{0}\right)}\left(\eta^{0}\right) \widetilde{T}_{-}^{\left(j, \mu_{0}\right)}\left(\eta^{0}\right) \neq 0
$$

Finally, we show Theorem B follows from Theorem C by using Theorem 3.

For simplicity, we restrict to the case when $l$ is odd. Then the above assertion easily follows from Theorem 3 and the following lemma.

Lemma 5.2. The following assertions hold.

$$
\sum_{j=1}^{2} T_{+}^{(j, 1)}\left(\eta^{0}\right) \widetilde{T}_{-}^{(j, 2)}\left(\eta^{0}\right)=0
$$

is equivalent to

$$
a=-\{2 n(l+1)+l+m\} \quad\left(n \in \mathbb{Z}_{+}, m=0,2\right) .
$$

$$
\sum_{j=1}^{2} T_{+}^{(j, 2)}\left(\eta^{0}\right) \widetilde{T}_{-}^{(j, 1)}\left(\eta^{0}\right)=0
$$

is equivalent to

$$
a=2 n(l+1)+l+m \quad\left(n \in \mathbb{Z}_{+}, \quad m=0,2\right) .
$$

Proof. Since the proofs are similar, we only prove (2) in the case $\eta_{0}>0$. From Theorem 3, (5.49) is equivalent to

$$
T_{+}^{(1,2)}\left(\eta^{0}\right) T_{+}^{(2,2)}\left(\eta^{0}\right)=0
$$

Now by (2.21), $T_{+}^{(1,2)}\left(\eta^{0}\right)$ and $T_{+}^{(2,2)}\left(\eta^{0}\right)$ are given by

$$
\left\{\begin{array}{l}
T_{+}^{(1,2)}\left(\eta^{0}\right)=\Lambda_{+}(1,2) \Gamma\left(\gamma_{2}+\delta_{2}+1\right) \sin \pi\left(\gamma_{2}+\delta_{2}\right) / \sin \pi\left(\gamma_{1}+\gamma_{2}+2 \delta_{2}\right), \\
T_{+}^{(2,2)}\left(\eta^{0}\right)=\left|\eta^{0}\right|^{-\delta_{2}} \Lambda_{+}(2,2) \Gamma\left(\gamma_{2}+1\right) \sin \pi \gamma_{2} / \sin \pi\left(\gamma_{1}+\gamma_{2}\right),
\end{array}\right.
$$

where $\Lambda_{+}(1,2), \Lambda_{+}(2,2)$ are nonvanishing and homogeneous of degree zero with respect to $\eta^{0}$. Here, from (5.12), we have

$$
\kappa_{i}=2^{-1}(l+1)^{-1}\left\{l+(-1)^{i} a\right\} \quad(i=1,2) \text {. }
$$

Hence, by (5.9) (5.52) and (5.53), we have

$$
\begin{aligned}
& T_{+}^{(1,2)}\left(\eta^{0}\right) T_{+}^{(2,2)}\left(\eta^{0}\right) \\
& \quad=\left|\eta^{0}\right|^{-\delta} \Lambda \Gamma\left(2^{-1}(l+1)^{-1}(l+a)\right) \Gamma\left(2^{-1}(l+1)^{-1}(l+a+2)\right)
\end{aligned}
$$

with 


$$
\begin{aligned}
& \Lambda=\Lambda_{+}(1,2) \Lambda_{+}(2,2) \sin \pi 2^{-1}(l+1)^{-1}(l-a) \\
& \times \sin \pi 2^{-1}(l+1)^{-1}(l-a+2) /\left(\sin \pi(l+1)^{-1} l \cdot \sin \pi(l+1)^{-1}(l+2)\right) .
\end{aligned}
$$

Recalling the well known formula $\Gamma(\rho) \Gamma(1-\rho)=\pi / \sin \pi \rho,(5.54)$ can be written in the form:

$$
\begin{aligned}
& T_{+}^{(1,2)}\left(\eta^{0}\right) T_{+}^{(2,2)}\left(\eta^{0}\right) \\
& \quad=\left|\eta^{0}\right|^{-\delta_{2}} \Lambda^{\prime} /\left(\Gamma\left(2^{-1}(l+1)^{-1}(l-a)\right) \Gamma\left(2^{-1}(l+1)^{-1}(l-a+2)\right),\right.
\end{aligned}
$$

where

$$
\Lambda^{\prime}=\pi^{2} \Lambda_{+}(1,2) \Lambda_{+}(2,2) /\left(\sin \pi 2^{-1}(l+1)^{-1} l \cdot \sin \pi 2^{-1}(l+1)^{-1}(l+2)\right) .
$$

Since $\Lambda_{+}(1,2), \Lambda_{+}(2,2)$ are nonzero, $\Lambda^{\prime}$ does not vanish. Thus the condition (5.51) is equivalent to

$$
-2^{-1}(l+1)^{-1}(l-a),-2^{-1}(l+1)^{-1}(l-a+2) \in Z_{+} .
$$

Clearly, (5.56) is equivalent to (5.50).

Q. E. D.

\section{References}

[1] Alinhac, S., Branching of singularities for a class of hyperbolic operators, Indiana Univ. Math. J., 27 (1978), 1027-1037.

[2] Amano, K., Branching of singularities for degenerate hyperbolic operators and Stokes' phenomena, Proc. Japan Acad., 56 (1980), 206-209.

[3] Amano, K. and Nakamura, G., Branching of singularities for degenerate hyperbolic operators and Stokes' phenomena II, Proc. Japan Acad., 57 (1981) 164-167.

[4] Boutet de Monvel, L., Hypoelliptic operators with double characteristics and related pseudodifferential operators, Comm. Pure Appl. Math., 27 (1974) 585-639.

[5] Chazarain, J., Operateurs hyperbolique a caracteristique de multiplicite constante, Ann. Inst. Fourier, 24 (1974) 137-202.

[6] Coddington, E. A. and Levinson, N., Theory of Ordinary Differential Equations, McGraw-Hill New York 1955.

[7] Hanges, N., Parametrices and propagation of singularities for operators with noninvolutive characteristics, Indiana Univ. Math. J., 28 (1979) 87-97.

[8] Hukuhara, M., Ordinary Differential Equations, Second edition, Iwanami, Tokyo 1980 (Japanese).

[9] Ivrii, V, Ya., Wave fronts of solutions of certain pseudo-differential equations, Funct. Anal. Appl., 10 (1976), 141-142.

[10] Kawai, T., Construction of local elementay solutions for linear partial differential operators with real analytic coefficients, Publ. RIMS, Kyoto Univ., 7 (1971-1972), 363-397.

[11] Kumanogo, H., Fundamental solution for a hyperbolic system with diagonal principal part, Comm. P. D. E., 4 (1979), 959-1015.

[12] Kumanogo, H. and Taniguchi K., Fourier integral operators of multi-phase and the fundamental solution for a hyperbolic system, Funkcialaj Ekvacioj, 22 (1979), 161-196.

[13] Nakamura, G. and Uryu, H., Parametrix of certain weakly hyperbolic operators, Comm. P. D. E., 5 (1980), 837-896.

[14] Nakane, S., Propagation of singularities and uniqueness in the Cauchy problem at a 
class of doubly characteristic points, Comm. P. D. E., 6 (1981), 917-927.

[15] Nishimoto, T., On a matching method for a linear ordinary differential equations containig a parameter I, Kodai Math. Sem. Rep., 17 (1965), 198-221.

[16] Oaku, T., A canonical form of a system of microdifferential equation with noninvolutory characteristic and branching of singularities, Proc. Japan Acad., 57 (1981), 205-209.

[17] Okubo, K., A global representation of a fundamental set of solutions and a Stokes' phenomenon for a system of linear ordinary differential equation, J. Math. Soc. Japan, 15 (1963), 268-288.

[18] Okubo, K. and Kohno, M., Asymptotic Expansion, Kyoiku-shuppan, Tokyo 1976 (Japanese).

[19] Shinkai, K., Fundamental solution of a degenerate hyperbolic system, Osaka J. Math., 18 (1981) 257-288.

[20] - Branching of singularities for a degenerate hyperbolic system, Comm. P. D. E., 7 (1982), 581-607.

[21] Takasaki, K., Singular Cauchy problem for a class of weakly hyperbolic differential operators (preprint).

[22] Taniguchi, K. and Tozaki, Y., A hyperbolic equation with double characteristics which has a solution with branching singularities, Math. Japonica, 25 (1980), 279-300.

[23] Treve, F., Discrete phenomena in uniqueness in the Cauchy problem, Proc. Amer. Math. Soc., 6 (1974), 229-233.

[24] Wasow, W., Asymptotic Expansion for Ordinary Differential Equations, Interscience 1965.

[25] Yoshikawa, A., Construction of a parametrix for the Cauchy problem of some weakly hyperbolic equation I, Hokkaido Math. J., 6 (1977), 313-344.

[26] Construction of a parametrix for the Cauchy problem of some weakly hyperbolic equation II, Hokkaido Math. J., 7 (1978), 1-26.

[27] - Construction of a parametrix for the Cauchy problem of some weakly hyperbolic equation III, Hokkaido Math. J., 7 (1978), 127-141. 
